\title{
An Efficient Method for the Design and Analysis of Microstrip Circuits and Antennas
}

\author{
by \\ Shailesh Raut
}

\begin{abstract}
A Thesis Presented to
The Faculty of Graduate Studies

In Partial Fulfillment of the Requirements for the Degree of Master of Science in Electrical Engineering

Department of Electrical and Computer Engineering University of Manitoba
\end{abstract}


The author has granted an irrevocable non-exclusive licence allowing the National Library of Canada to reproduce, loan, distribute or sell copies of his/her thesis by any means and in any form or format, making this thesis available to interested persons.
L'auteur a accordé une licence irrévocable et non exclusive permettant à la Bibliothèque nationale du Canada de reproduire, prêter, distribuer ou vendre des copies de sa thèse de quelque manière et sous quelque forme que ce soit pour mettre des exemplaires de cette thèse à la disposition des personnes intéressées.

L'auteur conserve la propriété du droit d'auteur qui protège sa thèse. Ni la thèse ni des extraits substantiels de celle-ci ne doivent être imprimés ou autrement reproduits sans son autorisation.

ISBN $\quad 0-612-16244-3$ 
Name SH+AILESH RAUT

Dissertation Abstracts International and Masters Abstracts International are arranged by broad, general subject categories.

Please select the one subject which most nearty describes the content of your dissertation or thesis. Enter the corresponding four-digit code in the spaces provided.

SUBJECT TERM

THE SCIENCES AND ENGINEERINE

\section{Subject Categories}

\section{THE HUMANITIES AND SOCIAL SCIENCES}

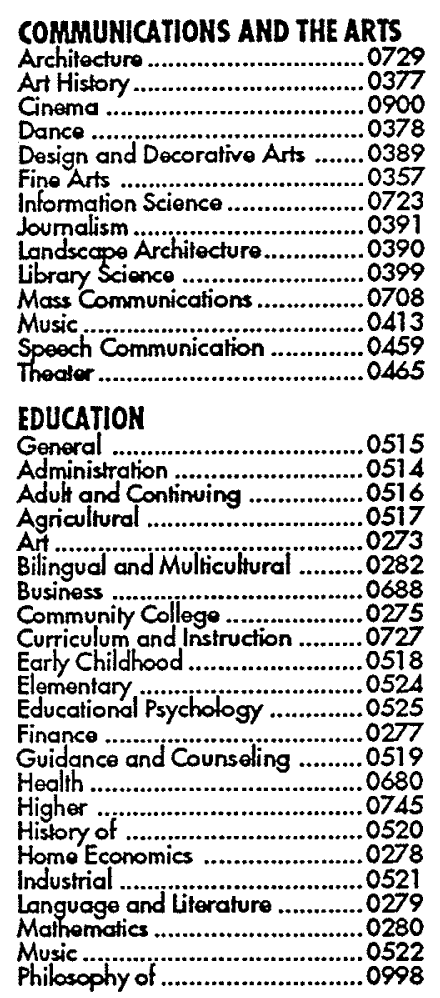

\section{THE SCIENCES AND ENGINEERING}

\section{BIOLOGICAL SCIENCES}

Agriculture

General ..............................0473

Animal Culture and

Nutrition

Animal Pahology ..................0476

Fisheries and Aquaculture .....0792

Food science and

Todnology and

Forestry and Wildifi.............0.0478

Plant Pathology .......................0480

Range Management ..............0777

Range Managemont ................0481
Soil Science .0...................048
Wood Technology ................0746

Biology

Goneral ...................................0306

Anatomy ………......................0287

Animal Physiobgy .................0433

Biostatistics ............................0308

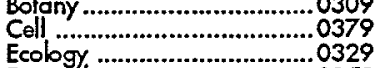

Ecology ................................. 0329

Genetics ................................0369

Limnology ...........................0793

Microbiology …...................0410

Molocular ...........................0307

Neuroscience ......................0317

Plant Physiology …….............0817

Volerinary Science .................0.0778

Biophysics

Meneral

EARTH SCIENCES

Biogeochemistry 0425

Geochemistiy

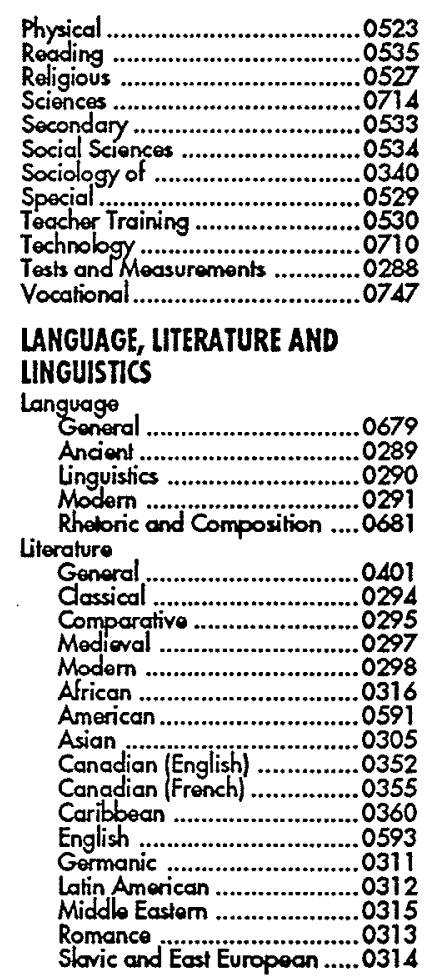

Goodesy .....................................0370

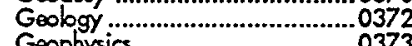
Geophysics..................................0373 Mineralogy .........................................0411 Paloobotany ......................................0345 Palobocolony …………....................0426 Paleontobgy …………………....0418

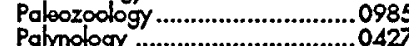
Physical Goography ………….......0368 Physical Ocoanography................0415

HEALTH AND ENVIRONMENTAL SCIENCES

Emvironmental Sciences ..............0768

Health Sciences 0566 Gonerd .................................0566 Audiology …...........................0300

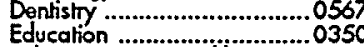
Administration, Hoalth Core 0769 Humen Dovelopment ............0758 Immunotogy …....................0982 Mediaine and Surgery ..........0564

Mental Health ......................0347

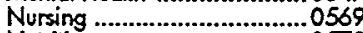

Nutrition .............................0570

Obstertics and Gynocology ..0380

Oecupationd Health and

Safety ..................................035

Oncology............................0992

Ophithalmology ......................038

Pahtology _.........................0571

Pharmacy ……..................0572

Public Hoalith.............................0573

Radiology …............................0574

Rehabilitaion and Therapy ...0382
Applied Sciences
$0,5 / 4 \sqrt{4} \mathrm{UMI}$ SUBJECT CODE
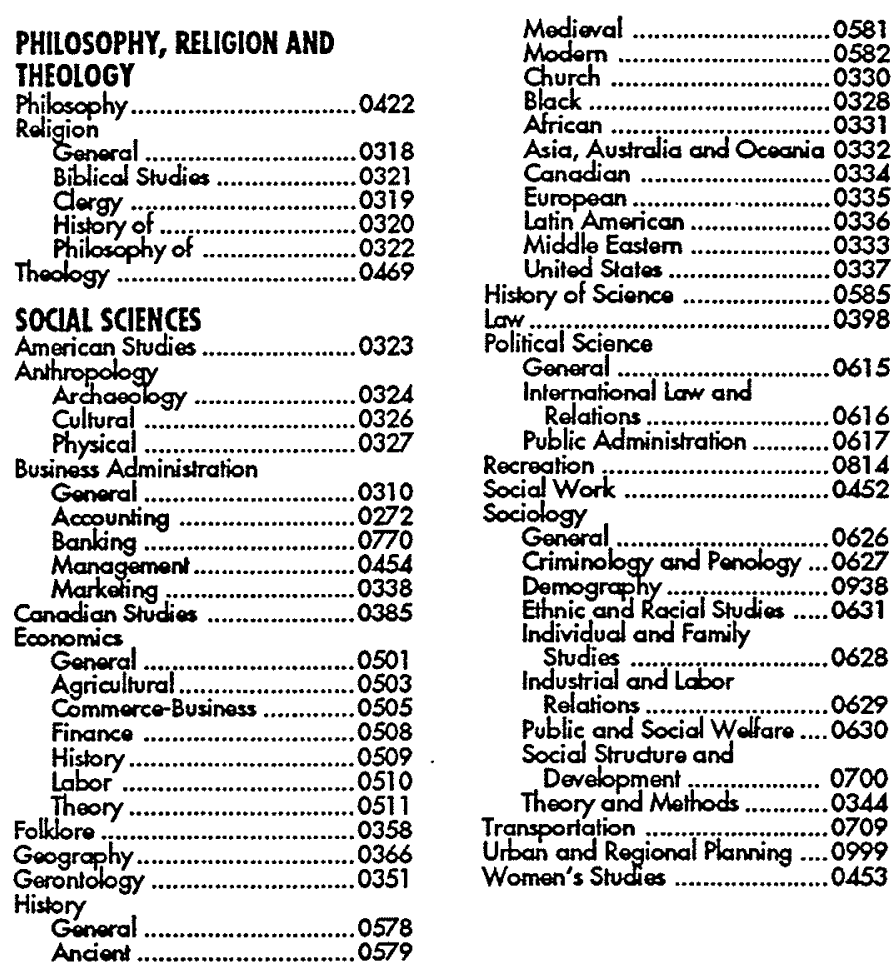

Sproch Pathology …..............0460 Home Economics ................................... 0383 PHYSICAL SAEHCES

\section{Pure Sciences}

Chemistry

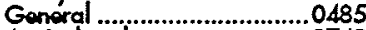

Agrialtural .............................0749

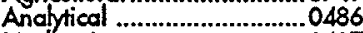

Biochemistry ..........................0487

Inorganic ............................0488

Organic ................................0490

Pharmocoutical ......................0491

Physical ..............................0494

Polymer .............................0495

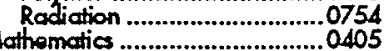

Physics

General ................................0605

Acoustics ............................0986

Astronomy and

Astrophysics ..................0606

Armospheric Science ...............0608

Atomic...............................0748

Condonsed Matter ...............0611

Electricity and Magnetism ....0607

Elementary Particles and

High Enargy ......................0798

Fluid and Plasma ....................0759

Molocular .............................0609

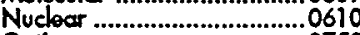

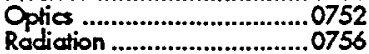

Statistias ……..................................0463

Applied Mechanics .....................0346

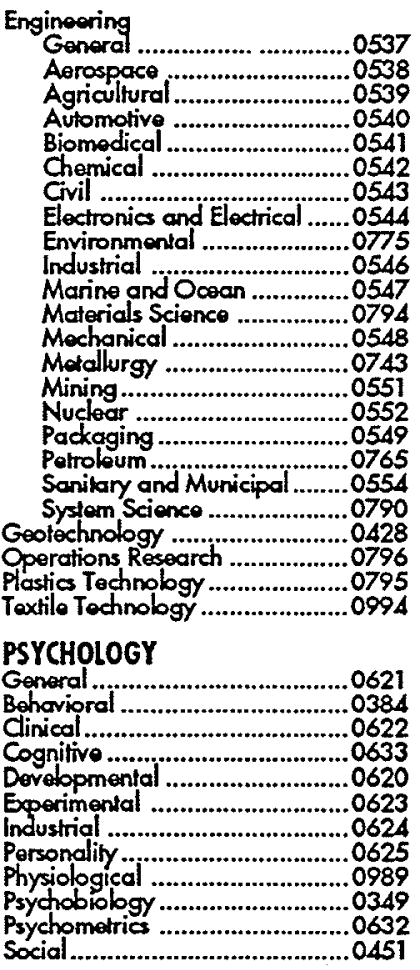




\section{FACULTY OF GRADUATE STUDTES}

THESIS/PRACTICUMI COPYRIGHT LICENSE

I, SHAILESHT RAUT

in the interest of facilitating research by others at The University of Manitoba (hereinafter referred to as the "Liversity") and elsewhere, and in satisfaction of a condition of being awarded a Master's or Doctoral degree as a graduate student at the University, hereby grant a license on the terms and conditions following to the University to reproduce my thesis/practicum, or any substantial part thereof, in any material form whatever, which thesis/practicum is to be submitted in partial fulfilment of the requirements for the degree of:

$$
\text { MASTER OF SCIENCE IN ELECTRICAL ENGINEERING }
$$

\section{Terms and Conditions}

1. This license shall continue for the full term of the copyright or for so long as may be legally permitted. 2. The Universal Copyright Notice shall be contained in reproductions and copies made under the authority of this license.

3. It shall be permissible for the University to submit reproductions and copies in any material form whatever to the National Library of Canada.

4. This license does not permit the sale of authorized reproductions and copies at a profit, but it does permit the collection by the University and the National Library of Canada of charges covering actual reproduction and copying costs.

5. All reproductions and copies made under the authority of this license shall bear a statement to the effect that the reproduction or copy in question has been made available by authority of the copyright owner solely for the purpose of private study and research and may only be reproduced and copied as permitted by copyright laws or with express written authorization from the copyright owner.

6. The effective date of this license will be the date that the thesis/practicum is submitted to the University. (In exceptional cases where adequate cause can be shown to delay reproduction and copying of the thesis/practicum, a request may be made to the Dean of Graduate Studies to restrict reproduction and copying of the thesis for a period of one year after its submission and the Dean, in consultation with the student's advisor, shall determine the period of restriction, if any.)

7. I save harmless and indemnify the University for any liability, losses, damages and aryards resulting from any actions, suits demands or claims, respecting an alleged or proven copyright infringement by the contents of my thesis/practicum or respecting defamatory statements contained within my thesis/practicum, and arising from the exercise by the University of its rights bereunder.

Signed and sealed this

day of

JANUARY

1997

\section{Student: $\quad$ SIAAiLESiH RAUT}

Student Vumber:

Witness:

(Seal)

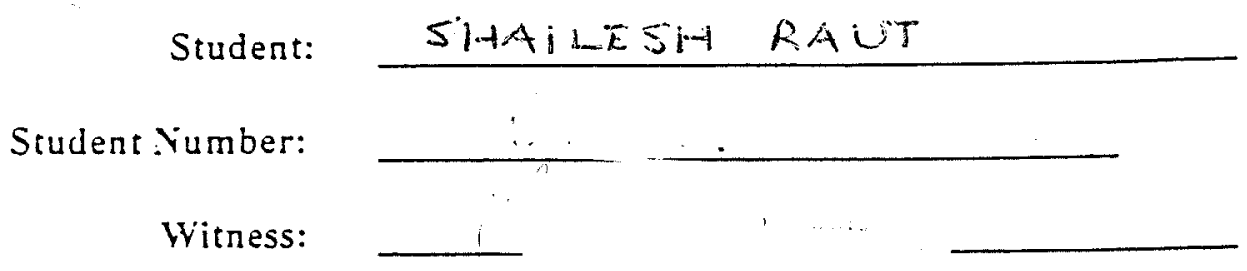




\title{
THE UNIVERSITY OF MANITOBA
}

FACULTY OF GRADUATE STUDIES

COPYRIGHT PERMISSION PAGE

\author{
AN EFFICIENT METHOD FOR THE DESIGN AND ANALYSIS OF \\ MICROSTRIP CIRCUITS AND ANTENNAS
}

BY

SHAILESH RAUT

A Thesis/Practicum submitted to the Faculty of Graduate Studies of The University of Manitoba in partial fulfillment of the requirements of the degree

of

MASTER OF SCIENCE

Shailesh Raut 1997 (c)

Permission has been granted to the Library of The University of Manitoba to lend or sell copies of this thesis/practicum, to the National Library of Canada to microfilm this thesis and to lend or sell copies of the film, and to Dissertations Abstracts International to publish an abstract of this thesis/practicum.

The author reserves other publication rights, and neither this thesis/practicum nor extensive extracts from it may be printed or otherwise reproduced without the author's written permission. 


\section{Abstract}

A dyadic Green's function based solution is studied and implemented to investigate the design characteristics of microstrip structures. This technqiue requires derivation of an integral equation solution in terms of vector and scalar potentials of the dyadic Green's function. The extraction of Green's function through Sommerfeld integral equations is avoided by enforcing instead the approximate Green's function. The reliability and efficiency of this approach is thoroughly examined and verified by comparing the solution with published results.

The final solution for the integral equation is acquired numerically by implementing the Method of Moments. A complete theoretical study of the numerical technique is presented. Surface current can be extracted from the solution and will provide a starting point for analysis of microstrip structures. Numerical results associated with the analysis of various microstrip lines and antennas are presented. Design and experimental results of a circularly polarized microstrip antenna are also provided. 


\section{Acknowledgements}

I wish to express my appreciation and gratitude to Dr. Sebak, for his guidance and support throughout the development of this project. I would also like to thank him for his encouragement and advice during the course of my graduate studies.

I would also like to show my appreciation to Dr. G. E. Bridges, Mr. Tom Judge and Dr. R. A. Said for their helpful hints and suggestions. I am grateful to all my friends and colleagues for making my University years more than just an academic experience. I would like to thank Peter Frank, Raj Kler and Richard Chan for their friendship and support. A very heartfelt thank you to Vanessa Acin for being there.

Finally, I wish to dedicate this thesis to my parents and my sister. Their love, support and understanding have helped me to realize all my dreams and ambitions. 


\section{TABLE OF CONTENTS}

Abstract

Acknowledgements ii

Table of Contents

List of Figures and Tables

List of Symbols

I. Introduction

1.1 Microstrip Transmission Lines

1.2 Microstrip Analysis Techniques $\quad$. . . . . . . . . . . . . . 3

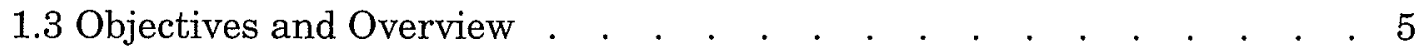

II. Mixed Potential Integral Equation and Dyadic Green's Function . . . 7

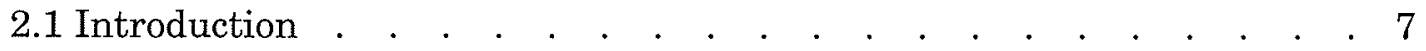

2.2 Mixed Potential Integral Equation _ . . . . . . . . . . . 7

2.3 Approximation of Dyadic Green's Function . . . . . . . . . . 11

III. The Method of Moments (MOM) and its Application to

Mixed Potential Integral Equation (MPIE) . . . . . . . . . . . . 16

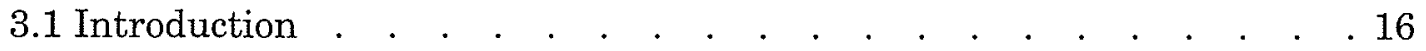

3.2 The Method of Moments . . . . . . . . . . . . . . . . 17

3.3 Transformation of MPIE by MOM . . . . . . . . . . . . . . 18

3.3.1 Basis Functions . . . . . . . . . . . . . . . . . 19

3.3.2 Equivalent Linear Equations for MPIE $\quad . \quad$. . . . . . . . . 23 
3.4 Matrix Setup and Numerical Considerations . . . . . . . . . 27

3.4.1 Y- Directed Currents . . . . . . . . . . . . . . . . 32

3.4.2 Programming Considerations . . . . . . . . . . . . . . . 34

IV. S - Parameters and Far - Field Radiation Pattern . . . . . . . . . . 37

4.1 Introduction . . . . . . . . . . . . . . . . . . . . 37

4.2 Frequency Dependent S - Parameters . . . . . . . . . . . 38

4.3 E- and H- Plane Radiation Patterns . . . . . . . . . . . . 41

V. Numerical Results . . . . . . . . . . . . . . . . . . . . 45

5.1 Introduction . . . . . . . . . . . . . . . . . . . . 45

5.2 Microstrip Transmission Lines and Dipoles . . . . . . . . . . 46

5.2.1 Microstrip Dipoles . . . . . . . . . . . . . . . . 46

5.2.2 Characteristic Impedance of Microstrip Lines . . . . . . . . 51

5.3 Microstrip Antennas . . . . . . . . . . . . . . . . . 54

5.3.1 Single Feed/Linearly Polarized Antenna . . . . . . . . . . 55

5.4 Circularly Polarized Microstrip Antenna $\quad . \quad$. . . . . . . . . . 64

5.4.1 Design Simulation and Experimental Results . . . . . . . 64

VI. Conclusion . . . . . . . . . . . . . . . . . . . . . .71

i. Bibliography . . . . . . . . . . . . . . . . . . . . 73

ii. Appendix A . . . . . . . . . . . . . . . . . . . . . . . 76

iii. Appendix B . . . . . . . . . . . . . . . . . . . . . 78 


\section{LIST OF FIGURES AND TABLES}

[1.1] Microstrip structure . . . . . . . . . . . . . . . . . . . . 3

[2.1] Microstrip structure with excitation $\quad . \quad$.

[3.1] Segmentation procedure for charge and current cells . . . . . . . . 20

[3.2] Current basis functions for $\mathrm{x}$-directed cells $\quad$. . . . . . . . . . . . . . 22

[3.3] Charge basis functions for $\mathrm{x}$-directed cells $\quad$. $\quad$. $\quad$. $\quad . \quad$. . . . . . . . 23

[3.4] Testing Functions (1-Dimensional rectangular pulse function) $\quad$. . . . . 26

[3.5] Delta-gap excitation for x-directed current cells . . . . . . . . . . . . 29

[3.6] Real and imaginary component of vector potential Green's function . . . 31

[3.7] x- and y-directed current cells for a microstrip conductor . . . . . . . 32

[3.8] Impedance calculation procedure for microstrip line $\quad . \quad$. . . . . . . . 36

[4.1] Simulation setup for a line fed microstrip antenna . . . . . . . . . . 38

[4.2] Transmission line model for a microstrip antenna $\quad . \quad$. . . . . . . . . . . 39

[5.1] Microstrip dipole with delta-gap excitation $\quad . \quad$. . . . . . . . . . . . 47

[5.2] Current magnitude for a single wavelength microstrip dipole . . . . . 48

[5.3] Phase distribution for single wavelength microstrip dipole . . . . . . . 48

[5.4] Input impedance of a microstrip line for different ground to dipole height $\quad .49$

[5.5] Input impedance vs. ground plane to dipole height . . . . . . . . . . 50

[5.6] Current distribution for off-center fed microstrip line . . . . . . . . . . 52

[5.7] Far field radiation pattern for off-center fed microstrip line . . . . . . . 52

[5.8] Segmentation for microstrip antenna simulation _ . . . . . . . . . . 56 
[5.9] Current distribution along the microstrip line . . . . . . . . . . 56

[5.10] Contour plot for the current distribution along the patch . . . . . . 57

[5.11] Normalized input impedance (Real) vs. frequency . . . . . . . . . 58

[5.12] Normalized input impedance (Imaginary) vs. frequency . . . . . . . 59

[5.13] E-plane radiation pattern for the microstrip antenna c . . . . . . 60

[5.14] H-plane radiation pattern for the microstrip antenna $\quad . \quad$. . . . . . . 60

[5.15] Segmentation for the antenna with matching transmission line feed $\quad . \quad 61$

[5.16] Reflection coefficients of the matched antenna . . . . . . . . . . 63

[5.17] Circularly polarized microstrip patch antenna . . . . . . . . . . 65

[5.18] Normalized input impedance of the antenna c . . . . . . . . .66

[5.19] Calculated reflection coefficient of the CP microstrip antenna . . . . . 67

[5.20] Measured reflection coefficient of the CP microstrip antenna . . . . . 68

[5.21] Far-field pattern at $2.5 \mathrm{GHz}$. . . . . . . . . . . . . . . .69

[5.22] Far-field pattern at $2.54 \mathrm{GHz}$. . . . . . . . . . . . . . . . . . . 69

Table [5.1] Characteristic Impedance of Off-Center Fed Line $\quad . \quad$. . . . . . . 53

Table [5.2] Run-time for MPIE/MOM solution . . . . . . . . . . . . . 63 


\section{LIST OF SYMBOLS}

$$
\begin{aligned}
& A\left(W b \cdot m^{-l}\right) \quad \text { Vector Magnetic Potential } \\
& c\left(m \cdot s^{-1}\right) \quad \text { Speed of light } \\
& E^{e}\left(V \cdot m^{-1}\right) \quad \text { Electric excitation field } \\
& E^{d}\left(V \cdot m^{-1}\right) \quad \text { Electric diffracted field } \\
& E_{t}\left(V \cdot m^{-1}\right) \quad \text { Tangential electric field } \\
& \vec{G}_{A}\left(\Omega \cdot s \cdot m^{-2}\right) \quad \text { Dyadic Green's fucntion for vector potential } \\
& G_{V}\left(F^{-1}\right) \quad \text { Dyadic Green's function for scalar potential } \\
& H^{e}\left(A \cdot m^{-l}\right) \quad \text { Magnetic exctiation field } \\
& H^{d}\left(A \cdot m^{-1}\right) \quad \text { Magnetic diffracted field } \\
& h(m) \quad \text { Height of the substrate } \\
& I_{x}(\mathrm{~A}) \quad \mathrm{X} \text { - directed surface current } \\
& J_{S}\left(A \cdot m^{-1}\right) \quad \text { Surface current density } \\
& k_{o}\left(m^{-1}\right) \quad \text { Free space wave number } \\
& L(f) \quad \text { Linear operator with response }
\end{aligned}
$$




\begin{tabular}{|c|c|}
\hline$T_{x}()$ & Current basis function (2-D rooftop function) \\
\hline$R($ ) & Charge basis function (2-D pulse function) \\
\hline$U()$ & Testing function (1-D pulse function) \\
\hline$V(V)$ & Scalar potential \\
\hline$\omega$ & angular frequency \\
\hline$\nabla_{t}$ & Tangential component of del operator \\
\hline$\rho_{s}$ & Surface charge density \\
\hline$\varepsilon_{r}$ & Relative permittivity \\
\hline$\varepsilon_{\text {eff }}$ & Effective relative permittivity \\
\hline$\lambda_{0}$ & Free space wavelength \\
\hline$\lambda_{g}$ & Guide wavelength \\
\hline$\mu_{o}$ & Permeability of vacuum \\
\hline$\Gamma_{A}, \Gamma_{V}$ & Notation for vector and scalar surface integ. \\
\hline
\end{tabular}




\title{
Chapter I
}

\author{
Introduction
}

\subsection{Microstrip Transmission Lines}

Over the past few decades, microstrip structures have gained considerable popularity in integrated circuit design. Major advantages such as efficient integration of active components and fairly compact size have attracted circuit designers to further explore the potentials of microstrip structures. This has resulted in a substantial increase in the application of various forms of microstrip transmission lines in different technological fields. Presently, their use is concentrated mostly in high frequency communication and microwave circuit design. Also, the same concept has been applied to design all kinds of printed circuits.

Demands for portable microwave circuitry for use in different applications, such as aerospace and satellite communications, led to the evolution of printed circuit design in early 1950s. Also, in the 1950s the concept of microstrip radiator was first 
proposed by Deschamps [8]. The first practical and fully operational microstrip antenna was designed by Howell and Munson. Further advancement and study of printed transmission lines resulted in the technology of Microwave Integrated circuits (MICs). Presently, the use of MICs as well as Microwave Monolithic Integrated Circuits (MMICs) is very popular among high frequency circuit designers [11].

The demand for analysis tools for practical microstrip structures such as printed circuit boards and integrated antennas is growing very fast. Due to the advancement in microstrip technology, researchers as well as industries try to look for the most efficient analysis approach. In the long run, their major concern is the efficiency of the analysis tool, i.e. program run-time and computer memory requirement. Therefore, an efficient tool with an accuracy of within few percentage is certainly more desirable than a tool which has a higher accuracy but requires large CPU time and memory. The main objective in circuit design these days is that as long as there is a good starting point in design specifications, modifications can be made faster experimentally. For most designers, this practical aspect is more important when considering economical advantages.

A microstrip structure, in its simplest form, comprises of two parallel conducting layers separated by a dielectric substrate. As shown in Fig. 1.1, the lower conductor functions as a ground plane and the upper conductor acts as the conducting line or radiator. These types of structures cannot support a pure TEM (Transverse Electromagnetic) wave due to the presence of the dielectric-air interface. Therefore, microstrip lines differ considerably from other transmission line structures such as the waveguide and coaxial line. The open configuration of microstrip structure makes it very convenient for use in integrated circuit design. 
Lumped elements such as transistors and resistors can be integrated on the same surface as the transmission line. This will drastically reduce the size of circuit boards and structures [11].

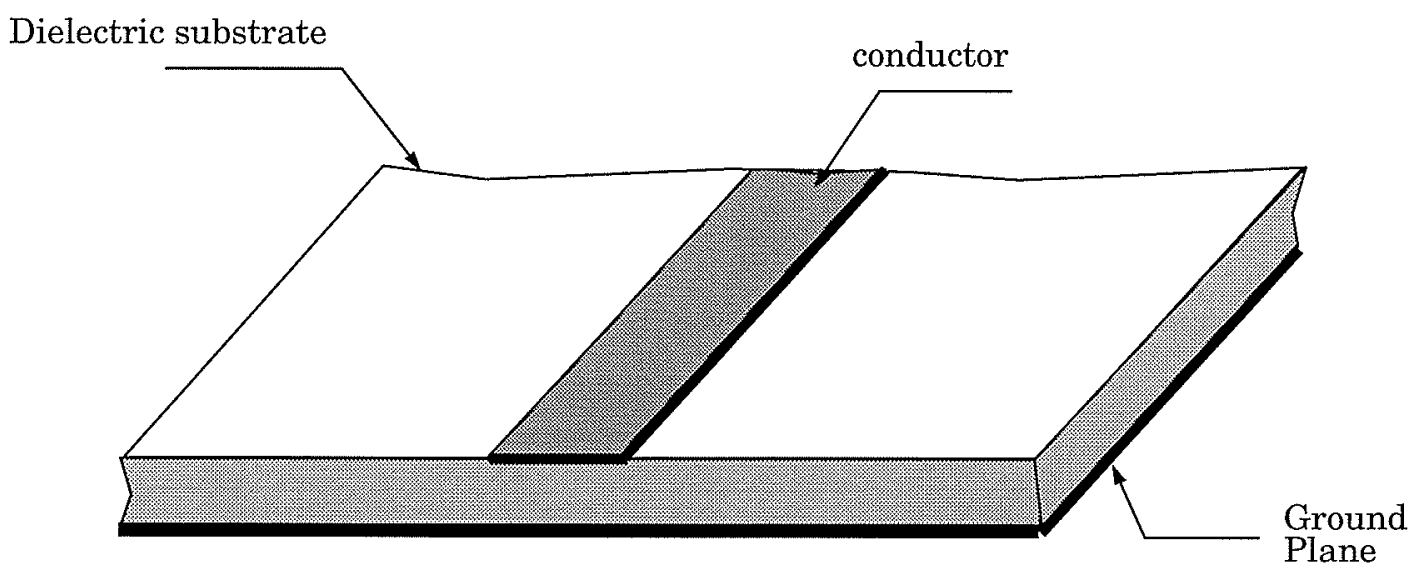

Figure. 1.1 : A simple microstrip Structure

Antennas based on the microstrip concept inherit the same properties as all microstrip structures. Microstrip antennas have considerable advantages over other types of conventional antennas. For example, because of their thin profile and lightweight structure, they can be easily integrated onto the structure of satellites, rockets and missiles. Microstrip antennas' popularity also lies in their compatibility with solid state devices, which can be fabricated, along with the feed lines, on the same substrate. However, they also suffer from some major disadvantages such as narrow bandwidth, poor isolation between the feed and the radiating element and poor endfire radiation. But, the advantages of microstrip antennas outweigh their disadvantages and furthermore, various techniques have been developed in order to counter these disadvantages [12]. Presently, there are numerous methods that can be used for the design and analysis of microstrip structures. An outline on the existing analysis methods is provided in the next section. 


\subsection{Microstrip Analysis Techniques}

There is a big demand for an accurate and efficient analysis method for microstrip structures. Over the years researchers have developed various numerical technqiues that can be applied to analyze microstrip structures. Presently, most of the microstrip analysis methods can be placed into three different categories. In the first category, the general microstrip characteristics are calculated from the electrostatic properties of the structure. These are known as the quasi-static methods and are adequate for designing circuits at low frequencies. The second category involves dispersion models, which takes into consideration the results obtained from either exact theoretical or experimental dispersion behaviour of the microstrip structure. In the final category, the hybrid nature of the mode of propagation is taken into consideration and is also known as the full wave analysis technique. Full wave analysis methods consider the complete field distribution of the microstrip structures and they require considerable computational and analytical efforts [6].

One of the most popular full wave analysis technique involves computation of integral equations by implementing Green's functions. The Green's functions are expressed in terms of Sommerfeld integrals which in turn form a solution to the wave equation in a defined medium [3]. Different numerical techniques can be implemented to solve and approximate the Sommerfeld integrals in order to extract the Green's function. This is generally a time consuming procedure. However, an approximation of the Green's function can be obtained through the Sommerfeld identity without the use of Sommerfeld integrals. With the absence of numerical integration, the calculation of Green's functions becomes very efficient and will translate to an efficient solution, both theoretically and numerically [5].

The use of an approximate Green's function is considered to be less reliable for 
both far field and near field analysis. Nevertheless this method can still be successfully implemented to analyze microstrip characteristics and will be the main objective of this thesis. Previous studies in Green's function solution by Yeung [2] and Mosig and Gardiol [1], [3] will be the foundation of this work. First, there are few reasonable assumptions that need to be considered in order to simplify the formulation of the method. The microstrip substrate is assumed to be homogenous, linear and isotropic and is assumed to extend to infinity in the transverse direction. The conductor itself is assumed to have zero thickness and considered a perfect electric conductor. Further assumptions are also introduced in the actual formulation and will be discussed in the relevant chapters.

\subsection{Objective and Overview}

The primary objective of this thesis is to validate the full-wave analysis method which implements an approximation method to extract Green's function instead of using the Sommerfeld integral technique. A computer program is written in Fortran to acquire the desired solutions. The numerical data obtained from our study is compared and verified with some published results. The solution is further criticized by comparing the data with a full-wave analysis software tool called PRELUDE [25]. Finally, a microstrip antenna is fabricated and analyzed to obtain certain design parameters and then compared with the results obtained from the numerical solution.

The thesis is presented in five different chapters. The second chapter focuses on conceptualizing the theory of Green's function and its integration into the Mixed Potential Integral Equation. A thorough derivation of the concept associated with the 
method is provided. Following this, in chapter 3, the integral equation will be completely transformed into a set of linear equations by implementing Method of Moments. Important considerations in both numerical as well as computational concepts will be explored thoroughly. In chapter 4, the theory associated with the extraction of S-parameters will be discussed, followed by a general derivation of the theory of far-field radiation pattern. Finally, the numerical data obtained from our solution will be compared and criticized with published results. Conclusions along with suggestions for improving the efficiency of the technique will be presented at the end of the thesis. 


\section{Chapter II}

\section{Mixed Potential Integral Equation and Dyadic Green's Function}

\subsection{Introduction}

The Mixed Potential Integral Equation technique is widely used in the analysis of wire antennas and has been successfully adapted to analyze microstrip structures [3]. A detailed description of the theory and application of the Mixed Potential Integral Equation (MPIE) is presented in this chapter. The dyadic Green's function used in the MPIE formulation will also be discussed in this chapter.

\subsection{Mixed Potential Integral Equation (MPIE)}

The MPIE has been extensively used in conjunction with the moment's method to analyze wire antennas. The modified MPIE formulation will be adapted in 
our work to analyze general microstrip structures [1]. Consider the microstrip structure shown in Fig. 2.1, where $C_{o}$ is the conducting patch on top of a grounded dielectric substrate of height $h$ and dielectric constant $\varepsilon_{1}$. The thickness of the top conductor is assumed to be zero for this case. The electric and magnetic excitation fields are denoted by $\boldsymbol{E}^{\boldsymbol{e}}$ and $\boldsymbol{H}^{\boldsymbol{e}}$, respectively.

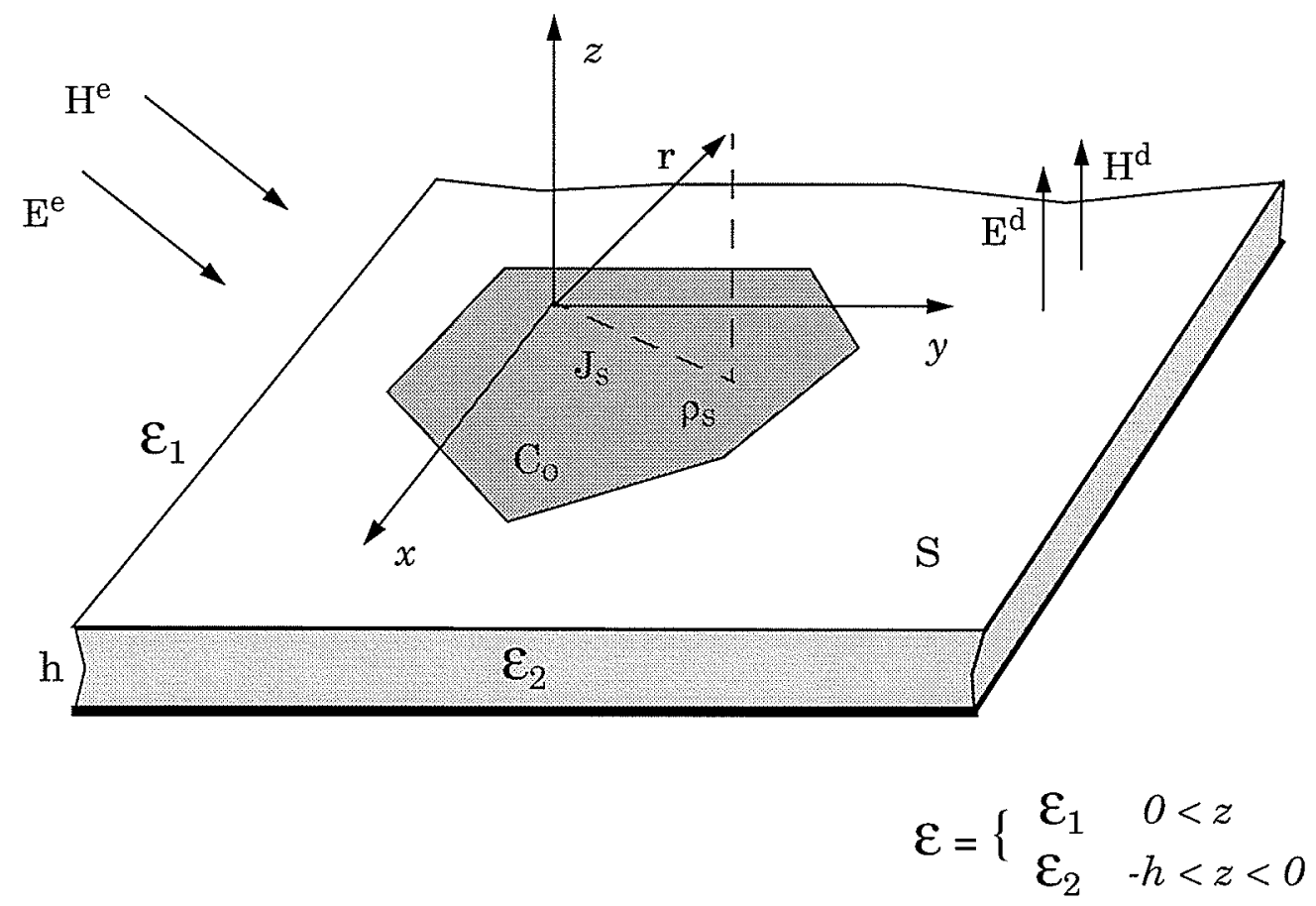

Figure 2.1 : Microstrip structure with excitation

The excitation fields can be created by a finite source, located within the conductor or can be the fields of a plane wave originating at infinity. Other excitation fields are also integrable to this structure, depending on the application. The surface charge density $\rho_{s}$ and surface current density $\boldsymbol{J}_{\boldsymbol{s}}$ are due to the introduction of a conducting plane within the excitation fields. Therefore, the total electromagnetic field is the sum of the excitation fields and the diffracted or scattered fields $\boldsymbol{E}^{\boldsymbol{d}}$ and 
$\boldsymbol{H}^{\boldsymbol{d}}$. The diffracted fields are created by the presence of charge and current densities.

The total electric field has to satisfy Maxwell's equations and the associated boundary conditions on the ground plane and the interface $S$. Therefore, assuming a perfect conductor, the boundary condition can be derived as,

$$
\begin{gathered}
\boldsymbol{e}_{z} \times \boldsymbol{E}^{\boldsymbol{t} o t(\boldsymbol{r})}=\boldsymbol{e}_{z} \times\left\{\boldsymbol{E}^{\boldsymbol{d}}(\boldsymbol{r})+\boldsymbol{E}^{\boldsymbol{e}}(\boldsymbol{r})\right\} \\
=\boldsymbol{E}_{t}^{\boldsymbol{d}}(\boldsymbol{r})+\boldsymbol{E}_{t}^{\boldsymbol{e}}(\boldsymbol{r})=0
\end{gathered}
$$

The magnetic vector potential $A$ can be such that,

$$
\boldsymbol{H}^{d}=\frac{1}{\mu} \nabla \times \boldsymbol{A}
$$

Now, from Maxwell's equation we have the following relationships

$$
\nabla \times \boldsymbol{E}=-j \omega \mu \boldsymbol{H}
$$

Substituting the magnetic potential, we obtain

$$
\nabla \times\left(\boldsymbol{E}^{d}+j \omega \boldsymbol{A}\right)=0
$$

By integrating the above equation and simplifying, the scattered field is given by,

$$
\boldsymbol{E}^{\boldsymbol{d}}=-j \omega \boldsymbol{A}-\nabla V
$$

Similarly, the divergence to the scalar potential $V$ is given by,

$$
\nabla \cdot \boldsymbol{A}=-j \omega \mu V
$$

The tangential component of the incident electric field is derived from the above equations as,

$$
\boldsymbol{E}_{\boldsymbol{t}}^{\boldsymbol{e}}=j \omega \boldsymbol{A}+\nabla V
$$

The scalar and vector potentials can be further expressed as superposition integrals of the corresponding Green's functions and the surface current density and surface charge density. Therefore : 


$$
\begin{aligned}
& \boldsymbol{A}(r)=\int_{s} \overrightarrow{\boldsymbol{G}}_{\boldsymbol{A}}\left(r \mid r^{\prime}\right) \cdot \boldsymbol{J}_{\boldsymbol{s}}\left(r^{\prime}\right) d r^{\prime} \\
& V(r)=\int_{s} G_{V}\left(r \mid r^{\prime}\right) \rho_{s}\left(r^{\prime}\right) d r^{\prime}
\end{aligned}
$$

In the above equation $\overrightarrow{\boldsymbol{G}}_{\boldsymbol{A}}$ and $G_{V}$ are the dyadic Green's functions for the vector potential and scalar potential respectively. Also, $r$ represents the location of the observation point and $r^{\prime}$ is the source point location. Solutions for the vector and scalar potentials created by $\mathrm{x}$ - directed horizontal electric dipole is the basis of MPIE formulation. $\overrightarrow{\boldsymbol{G}}_{\boldsymbol{A}}\left(r \mid r^{\prime}\right)$ is a dyadic function and it consists basically of nine terms which derives from,

$$
\vec{G}_{A}=e_{x} G_{A}^{x}+e_{y} G_{A}^{y}+e_{z} G_{A}^{z}
$$

Further simplification gives,

$$
\begin{gathered}
\overrightarrow{\boldsymbol{G}}_{\boldsymbol{A}}=\boldsymbol{e}_{\boldsymbol{x}}\left(\boldsymbol{e}_{\boldsymbol{x}} G_{A}^{x x}+\boldsymbol{e}_{\boldsymbol{y}} G_{A}^{x y}+\boldsymbol{e}_{\boldsymbol{z}} G_{A}^{x z}\right)+\boldsymbol{e}_{\boldsymbol{y}}\left(\boldsymbol{e}_{\boldsymbol{x}} G_{A}^{y x}+\boldsymbol{e}_{\boldsymbol{y}} G_{A}^{y y}+\boldsymbol{e}_{\boldsymbol{z}} G_{A}^{y z}\right)+ \\
\boldsymbol{e}_{\boldsymbol{z}}\left(\boldsymbol{e}_{\boldsymbol{x}} G_{A}^{z x}+\boldsymbol{e}_{\boldsymbol{y}} G_{A}^{z y}+\boldsymbol{e}_{\boldsymbol{z}} G_{A}^{z z}\right)
\end{gathered}
$$

In the above equation, since there is no z-directed current, because of the absence of vertical component in microstrip structures $x z, y z$ and $z z$ components are neglected. Also, the xy and yx components vanish and through the MPIE solution, where only tangential fields are considered, $\mathrm{zx}$ and $\mathrm{zy}$ components will not be implemented in the solution. Therefore, only two components, $x x$ and yy, need to be considered. By substituting the dyadic Green's function in the tangential component of electric field given in equation 2.8 , we obtain

$$
\boldsymbol{E}_{\boldsymbol{t}}^{\boldsymbol{e}}(\boldsymbol{r})=j \omega \int_{s} \overrightarrow{\boldsymbol{G}}_{\boldsymbol{A}}\left(r \mid r^{\prime}\right) \cdot \boldsymbol{J}_{\boldsymbol{s}}\left(r^{\prime}\right) d r^{\prime}+\nabla_{t} \int_{s} G_{V}\left(r \mid r^{\prime}\right) \rho_{s}\left(r^{\prime}\right) d r^{\prime}
$$

Furthermore, the scalar term can be derived in terms of surface current 
density by implementing the continuity equation.

$$
\rho_{s}=-\frac{l}{j \omega}\left(\nabla_{t} \cdot \boldsymbol{J}_{s}\right)
$$

Therefore, the MPIE is given as,

$$
\boldsymbol{E}_{\boldsymbol{t}}^{\boldsymbol{e}}(\boldsymbol{r})=j \omega \int_{s} \overrightarrow{\boldsymbol{G}}_{\boldsymbol{A}}\left(r \mid r^{\prime}\right) \cdot \boldsymbol{J}_{\boldsymbol{s}}\left(r^{\prime}\right) d r^{\prime}+\nabla_{t} \int_{s} G_{V}\left(r \mid r^{\prime}\right) \nabla_{t} \cdot \boldsymbol{J}_{\boldsymbol{s}}\left(r^{\prime}\right) d r^{\prime}
$$

The above MPIE expression is used for the solution of all general microstrip structures. The dyadic Green's functions can be derived from either Sommerfeld integrals or from it's approximate solution. Our objective in this work is to implement an approximation method to extract Green's function components that can be implemented in the MPIE. This approach will increase the efficiency of the solution. In the above formulation, current density is the only unknown component and a numerical technique will be applied for its' solution. As will be explained later, the Method of Moments is considered to solve the MPIE. This method basically involves transforming the above integrals into a simple set of linear equations and calculating the unknown (currents). The method will be discussed in the following chapter.

\subsection{Approximation of Dyadic Green's Function}

The basic definition states that the Green's functions are actually the potentials created by unit sources located within a surface. Once the impulse response, i.e. Green's function, is known, the fields produced by any current distribution can be easily determined by superposition. This is possible with Dirac delta sources located throughout the whole surface, which in limit reduces to the integral equation as given above in equation (2.15).

As mentioned previously, the dyadic Green's functions for open microstrip 
structures are obtained through the Sommerfeld integrals [4]. Since the derivation of Sommerfeld integrals is beyond the scope of our thesis, it will not be discussed here. The computation of Sommerfeld integrals is not only time consuming but also requires a large computation memory space. The solution is considerably demanding in terms of run time and memory. Furthermore, this complexity grows as the structure to be analyzed becomes larger. To avoid these problems, an approximate form of spatial dyadic Green's function can be implemented in the MPIE solution to obtain the design characteristics of microstrip structures.

The Green's function is represented by a combination of three different parts. First part consists of a quasi-dynamic images, dominant in the near field region. The second part represents the contribution of complex images, which are dominant in the intermediate region and the final part represents the contribution of surface waves dominating in the far field region of the substrate. The combination of these three parts represents the closed form solution to the dyadic Green's function. Among the three regions, the near field approximation is most dominant and it substantially influences the outcome of the final field solution [5]. The remaining two parts will be briefly discussed below. We know that,

$$
\begin{aligned}
G_{A}^{x x} & =G_{A o}^{x x}+G_{A, c i}^{x x}+G_{A, s w}^{x x} \\
G_{V} & =G_{V o}+G_{V, c i}+G_{V, s w}
\end{aligned}
$$

In the above equation, the vector and scalar Green's function associated with the intermediate region is given as [5],

$$
\begin{gathered}
G_{A, c i}^{x x}=\frac{\mu_{o}}{4 \pi} \sum_{i=1}^{N} a_{i} \frac{\exp \left(-j k_{o} r_{i}\right)}{r_{i}} \\
G_{V, c i}=\frac{1}{4 \pi \varepsilon_{o}} \sum_{i=1}^{N} a_{i}^{\prime} \frac{\exp \left(-j k_{o} r_{i}^{\prime}\right)}{r_{i}^{\prime}}
\end{gathered}
$$


where $r_{i}, r_{i}^{\prime}$ are the complex distances and $a_{i}, a_{i}^{\prime}$ are the amplitudes given by,

$$
a_{i}=A_{i} e^{\frac{B_{i} T_{o}}{I+j T_{o}}}
$$

Similarly, the vector and scalar Green's functions which contribute to the surface waves are given by [5]

$$
\begin{gathered}
G_{A, s w}^{x x}=\frac{\mu_{o}}{4 \pi}(-2 \pi j) \operatorname{Res}_{1} H_{o}^{(2)}\left(k_{\rho \rho} \rho\right) k_{\rho \rho} \\
G_{V, s w}=\frac{1}{4 \pi \varepsilon_{o}}(-2 \pi j) \operatorname{Res}_{2} H_{o}^{(2)}\left(k_{\rho \rho} \rho\right) k_{\rho \rho}
\end{gathered}
$$

In above relations, $k_{\rho \rho}$ is the surface wave pole which is located on the real axis of the complex $k_{\rho}$ plane. Also, Res is the residue of the corresponding integrand at the pole $k_{\rho}=k_{\rho \rho}$ and given by

$$
\begin{gathered}
\operatorname{Res}_{1}=\sum_{\rho(T E)}\left[\frac{\exp \left(-j k_{z o}\left(z+z^{\prime}\right)\right)}{j 2 k_{z o}}\right]_{k_{\rho}=k_{\rho \rho}} \lim \left(k_{\rho}-k_{\rho \rho}\right) R_{T E} \\
R e s_{2}=\sum_{\rho(T E, T M)}\left[\frac{\exp \left(-j k_{z o}\left(z+z^{\prime}\right)\right)}{j 2 k_{z o}}\right]_{k_{\rho}=k_{\rho \rho}} \lim \left(k_{\rho}-k_{\rho \rho}\right)\left(R_{T E}+R_{Q}\right)
\end{gathered}
$$

where,

$$
\begin{gathered}
R_{T E}=\frac{-\left(r_{10}^{T E}+e^{-j 2 k} z^{h}\right)}{1+r_{10}^{T E} e^{-j 2 k} z^{h}} \\
R_{Q}=\frac{2 k_{z o}^{2}\left(1-\varepsilon_{r}\right)\left(1-e^{-j 2 k} z^{h}\right)}{\left(k_{z I}+k_{z o}\right)\left(k_{z l}+\varepsilon_{r} k_{z o}\right)\left(1+r_{10}^{T E} e^{-j 2 k} z^{h}\right)\left(1+r_{10}^{T M} e^{-j 2 k} z^{h}\right)} \\
R_{T E} \text { and } R_{Q} \text { take into consideration the properties associated with }
\end{gathered}
$$

microstrip substrate. The final solution for the surface wave Green's functions can be 
obtained from complex integrals. As mentioned before, this term however is not very critical in the calculation of current distribution and near field solutions. We will not further investigate the surface wave and intermediate region solutions. Therefore, the above mentioned Green's function associated with the two regions will not be implemented in our analysis. Reference [5] provides the necessary derivations related to the vector and scalar potential Green's functions dominant in the far field and intermediate field.

In actual calculations, for most practical microstrip structures, the contribution of the quasi-dynamic images, which are dominant in the near field, is sufficient. This is valid in most microstrip circuits and was studied rigorously by Mosig and Gardiol [1]. Furthermore, the solution can be manipulated to obtain the far-field properties of microstrip structures. In our MPIE solution, the quasi-static approximation of Green's function will be implemented to obtain surface currents and far field radiation patterns. The solution will be verified by comparing it to the published results [2], [4], [7] as well as results obtained from an existing full-wave analysis tool. As stated before, this is the main focus of our study.

The vector and scalar components of the near field approximation of dyadic Green's function are given by,

$$
\begin{gathered}
G_{A}^{x x}\left(r \mid r^{\prime}\right)=\frac{\mu_{o}}{4 \pi}\left[\frac{\exp \left(-j k_{o} r_{o}\right)}{r_{o}}-\frac{\exp \left(-j k_{o} r_{1}\right)}{r_{1}}\right] \\
G_{V}\left(r \mid r^{\prime}\right)=\frac{1-\eta}{4 \pi j \omega \varepsilon_{o}}\left[\frac{\exp \left(-j k_{o} r_{o}\right)}{r_{o}}-(1+\eta) \sum_{i=1}^{\infty} \frac{\exp \left(-j k_{o} r_{i}\right)}{r_{i}}\right]
\end{gathered}
$$

where,

$$
\begin{aligned}
& r_{i}^{2}=\rho^{2}+(z+2 i h)^{2} \\
& \eta=\frac{\left(\varepsilon_{r}-l\right)}{\left(\varepsilon_{r}+l\right)}
\end{aligned}
$$


From the above approximate equations we can observe that the vector potential does not depend on the substrate permittivity. The expressions are related to image theory, in which the reflections from ground plane are accounted for by virtual sources. The real source is approximated by the first exponential term and the image source is approximated by the second term.

In our study the numerical integration of Sommerfeld integrals will be completely avoided and instead the estimated near field approximations of the vector and scalar Green's functions will be used in the MPIE . Our work will be compared with the studies conducted by Yeung [2] and Couture [4] to show that these approximated solutions are as effective as the solutions obtained by implementing Sommerfeld integrals. The following chapter focuses on the formulation associated with the application of the method of moments to the MPIE. 


\section{Chapter III}

\section{The Method of Moments and its \\ Application to Mixed Potential Integral Equation}

\subsection{Introduction}

The Method of Moments is generally used in the calculation of integral equations. its use in electromagnetics was first thoroughly explored by Harrington [10] and has been one of the more popular techniques for solving integral equations. The main focus of this chapter is to understand the theory associated with the transformation of Mixed Potential Integral Equation (MPIE) into linear equations by implementing the Method of Moments (MOM). General properties of the MOM will be discussed in the next section followed by a thorough explanation of basis and testing functions. To make the final solution more efficient, we will introduce some programming techniques in the algorithm. Some of the important methods that can substantially reduce the run time are presented at the end. 


\subsection{The Method of Moments}

The Method of Moments (MOM) has been frequently used in applied electromagnetics and high frequency problems for solving complex integral equation based problems. The main feature of this method is that it can transform differential equations (or integrals) to a set of linear equations by expanding the unknown variables into a set of specific functions. The approximate solution can be obtained by applying an error limiting process [10].

To clarify this method, consider an inhomogeneous function

$$
L(f)=g
$$

In the above equation $L$ is the linear operator, $f$ is the response (unknown) and $g$ is the excitation coefficient (known). Since $f$ can be expanded in a series of functions $f_{1}, f_{2}, f_{3}, \ldots$ in the domain of $L$, we have,

$$
f=\sum_{n} \alpha_{n} f_{n}
$$

where $\alpha_{n}$ are the expansion coefficients. For exact solution of $f$ the summation is usually infinite and $f_{n}$ will form a complete set of basis functions. However, finite summation will provide an approximate solution for $f$, which will then consist of a finite number of $f_{n}$. Therefore, by substitution we get

$$
\sum_{n} \alpha_{n} L\left(f_{n}\right)=g
$$

Assuming an inner product $\langle f, g\rangle$ has been determined for the problem, we can define a set of weighting (testing) functions $w_{1}, w_{2}, w_{3} \ldots$ in the range of $\mathrm{L}$. Taking the inner product of the above function with each $w_{n}$, we obtain, 


$$
\sum_{n} \alpha_{n}\left\langle w_{m}, L\left(f_{n}\right)\right\rangle=\left\langle w_{m}, g\right\rangle
$$

where, $m=1,2,3, \ldots M$ and $n=1,2,3, \ldots N$. Now, this set of equations can be written in a matrix form as,

$$
\left[l_{m n}\right]\left[\alpha_{n}\right]=\left[g_{m}\right]
$$

where,

$$
\left[l_{m n}\right]=\left[\begin{array}{ccc}
\left\langle w_{I}, L\left(f_{I}\right)\right\rangle & \ldots & \ldots \\
\ldots & \ldots & \ldots \\
\ldots & \ldots & \left\langle w_{M}, L\left(f_{N}\right)\right\rangle
\end{array}\right] \text { and }\left[g_{m}\right]=\left[\begin{array}{c}
\left\langle w_{I}, g\right\rangle \\
\ldots \\
\left\langle w_{M}, g\right\rangle
\end{array}\right]
$$

If the above matrix is non-singular, the inverse of $\left[l_{m n}\right]$ can be easily obtained. Therefore, the unknown response $f$ can be calculated :

$$
f=\left[\tilde{f}_{n}\right]\left[\alpha_{n}\right]=\left[\tilde{f}_{n}\right]\left[l_{m n}\right]^{-1}\left[g_{m}\right]
$$

where $\left[\tilde{f}_{n}\right]=\left[f_{1}, f_{2}, f_{3}, \ldots\right]$

As explained previously, increasing the matrix dimension improves the solution. The accuracy of this method is also directly affected by the choice of basis and testing functions.

\subsection{Transformation of MPIE by MOM}

The MPIE method described in Ch. 2 will now be implemented to the analysis of general microstrip structures. In short, the procedure involves transforming the MPIE into a system of linear equations through the application of MOM. This will involve specifying basis and testing functions to expand the integral equations. To 
simplify the analysis, we will introduce the theory in two subsections : in the first part, the basis functions will be discussed followed, in the second part, by a thorough derivation of the MPIE to linear equation transformation.

\subsubsection{Basis Functions}

First of all, before deciding the type of basis functions to be used, the microstrip structure to be examined is discretized into small rectangular/square segments called charge segments. Despite the original shape of the microstrip structure, which can be either rectangular or can assume any shape, its' region can be segmented into charge cells of equal dimension. It is important for the analysis that the charge cells be of equal size, which is critical in order to minimize the complexity of the MPIE solution. But this can be modified when analyzing structures with varying dimensions. In our case rectangular charge cells are considered.

The selection of basis functions is one of the keys to obtaining satisfactory results from MOM. Basis functions should be linearly independent so that $f$, given above in equation 3.7 , can be approximated reasonably. Subdomain basis functions are used for our case mainly because no assumptions are needed for current distributions over the conductor. The $2-\mathrm{D}$ rooftop function and 2-D pulse function are used as the basis functions for the expansion of surface current and charge density, respectively. The use of basis and testing functions can be further clarified by an example, provided below.

Consider a rectangular microstrip conductor of length $l$ and width $w$, as shown in Fig 3.1. To make the explanation simpler, assume that the y-component of the current is negligible because the width is assumed to be very small. However, the y- 
directed current will be taken into consideration in the final calculation procedure. The physical derivation is quite similar to the $\mathrm{x}$-directed current and it will be discussed in the end sections. For now, we will assume that the conductor only supports x-directed currents.

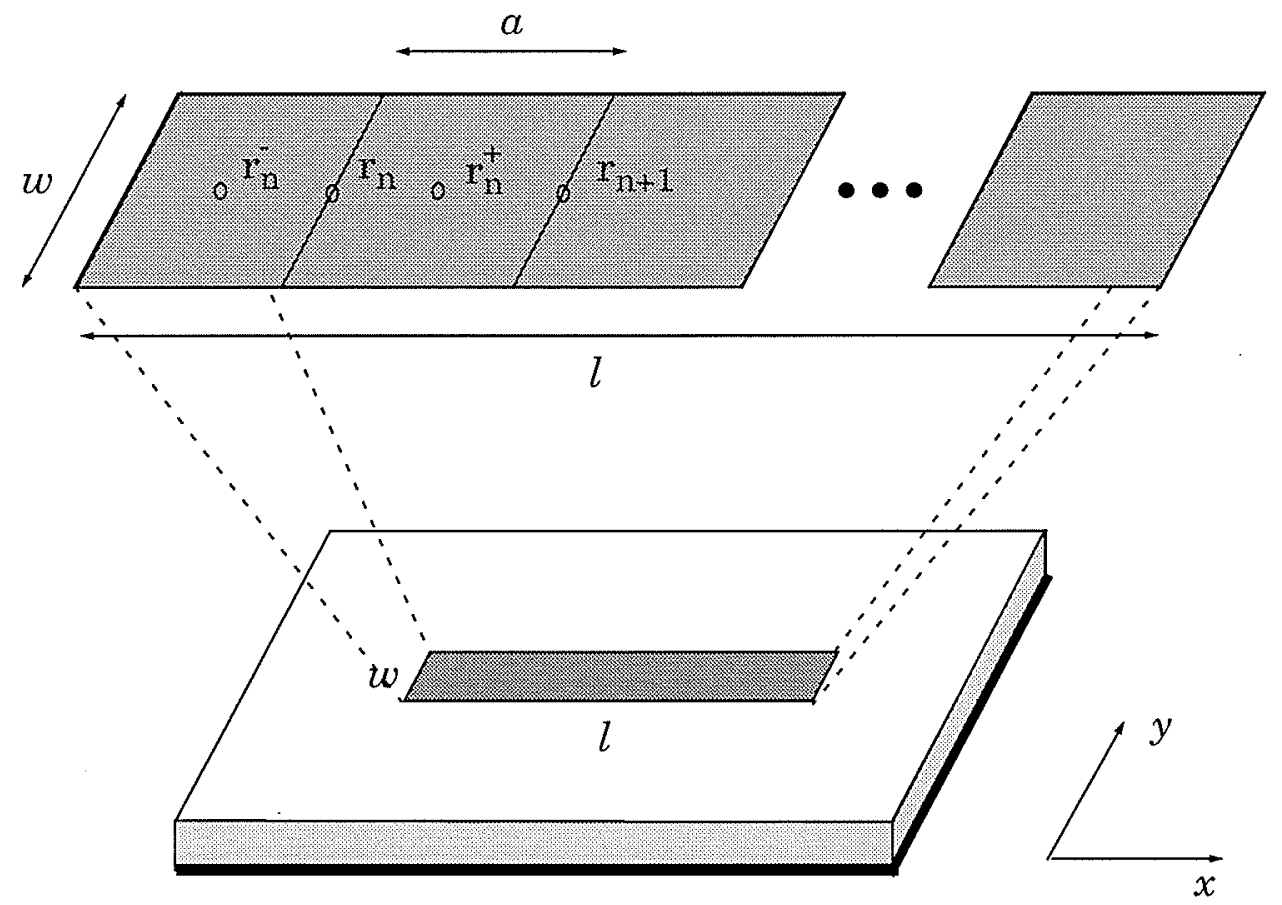

Figure 3.1 : Segmentation procedure for charge and current cells

The patch is segmented into $\mathrm{N}$ charge cells having equal dimensions of length $a$ and width $w$. Two adjacent charge cells, which share a common border along $\mathrm{x}-$ direction, will form one current cell having dimension of $(2 a \times w)$. This will result in a situation where one charge cell may contribute to the formation of four different current cells, when considering the cell division along the y-direction. Since a pair of charge cells constitute a current cell, there will be an overlapping of current cells. In the figure, $r_{n}$ is the center of individual current cell. Also, $r_{n}{ }^{+}$and $r_{n}{ }^{-}$are the centers of the adjacent charge cells which make up the current cell. 
The number of current cells is directly related to the number of charge cells. For example, for a conductor strip with $M$ charge cells, the number of current cells is $(M-1)$. But this might also depend on the shape of the conductor.

Each of these current cells support one basis function, as shown in Fig.3.2. For the analysis of microstrip structures, the use of a rooftop function (2-D triangular function) as the basis function is more suitable [2]. The expansion of current density distribution by $2-\mathrm{D}$ triangular function is given by [3],

$$
\boldsymbol{J}_{s}=\frac{1}{w} \sum_{n=1}^{N-1} I_{x n} T_{x}\left(r-r_{n}\right)
$$

where $I_{x n}$ is the current associated with each cell and $T_{x}\left(r-r_{n}\right)$ is the 2-D triangular function. The introduction of $\frac{1}{w}$ in the expansion above yields an unknown current coefficient having dimension of a current. Furthermore, every current coefficient gives the total current located within the individual current cells and the current flowing across the boundary of two charge cells.

$$
T_{x}\left(r-r_{n}\right)= \begin{cases}1-\frac{|x|}{a} & |x|<a, \quad|y|<\frac{w}{2} \\ 0 & \text { elsewhere }\end{cases}
$$

Fig. 3.2 shows the actual distribution of basis functions over the conductor. Since the current cells overlap each other, the same is true for the basis functions because each current cell supports a single basis function. 


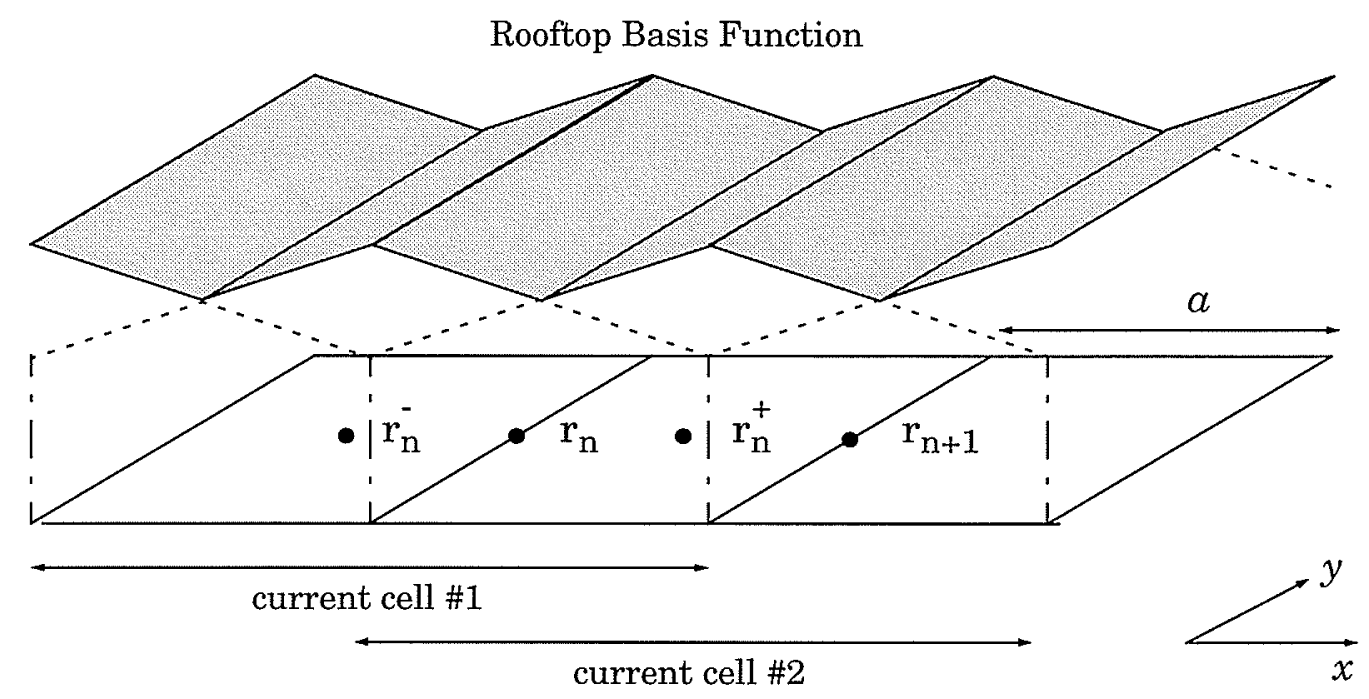

Figure 3.2 : Current basis functions for $x$-directed cells

The surface charge density $\rho_{s}$ can be obtained by using the continuity equation to the expansion of $\boldsymbol{J}_{\boldsymbol{s}}$, as described in Ch. 2. The notation $b$ will be used for the width of the microstrip line.

$$
\begin{aligned}
\rho_{s} & =-\frac{1}{j \omega}\left(\nabla_{t} \cdot \boldsymbol{J}_{s}\right) \\
& =-\frac{1}{j \omega} \frac{d}{d x}\left[\frac{1}{b} \sum_{n=1}^{N-1} I_{x n} T_{x}\left(r-r_{n}\right)\right] \\
& =\frac{1}{j \omega a b} \sum_{n=1}^{N-1} I_{x n}\left[R\left(r-r_{n}^{+}\right)-R\left(r-r_{n}{ }^{-}\right)\right]
\end{aligned}
$$

where, $R(r)$ is a two dimensional unit pulse function situated over a single charge cell.

$$
R\left(r-r_{n}\right)= \begin{cases}1 & |x|<\frac{a}{2}, \quad|y|<\frac{b}{2} \\ 0 & \text { elsewhere }\end{cases}
$$

The charge density within each charge cell remains constant and is made up 
of at least two components from the two adjacent current cells.

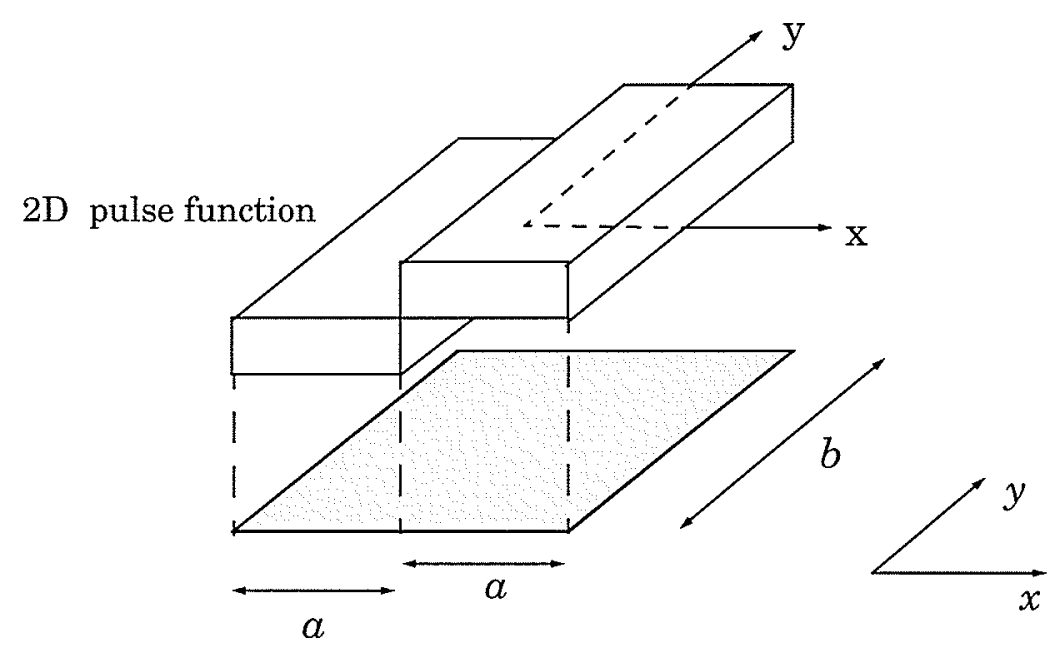

Figure 3.3 : Charge basis functions for $x$-directed cells

The concept is shown in Fig. 3.3, where the distribution of charge basis function is located within the adjacent charge cells associated with an individual current cell. This is true throughout the distribution of surface charge cells except at the end cells, where the charge cell contributes to the formation of only one current cell. The charge density is discontinuous on the borders between the adjacent charge cells and a singularity situation may arise. Therefore, a testing function must be selected carefully in order to avoid the points where the electric field becomes singular. The selection of testing function will be discussed in the next section, which describes the final transformation of MPIE to linear equations.

\subsubsection{Equivalent Linear Equations for MPIE}

The final step now is to introduce a testing function in the MPIE/MOM formulation. As mentioned before, the testing function must be compatible with the basis functions. Once again, for simplicity we are still considering only $x$ - directed 
currents and therefore all the tangential components are x- directed. Going back to the tangential component of the electric field, from equation 2.13 ,

$$
\begin{aligned}
\boldsymbol{E}_{\boldsymbol{x}}^{\boldsymbol{e}}(\boldsymbol{r}) & =j \omega \int_{s} \vec{G}_{A}^{x x}\left(r \mid r^{\prime}\right) \cdot \boldsymbol{J}_{\boldsymbol{x}}\left(r^{\prime}\right) d r^{\prime}+\nabla_{t} \int_{s} G_{V}\left(r \mid r^{\prime}\right) \rho_{x}\left(r^{\prime}\right) d r^{\prime} \\
& =j \omega \int_{s} \vec{G}_{A}^{x x}\left(r \mid r^{\prime}\right) \cdot \boldsymbol{J}_{\boldsymbol{x}}\left(r^{\prime}\right) d r^{\prime}+\frac{d}{d x} \int_{s} G_{V}\left(r \mid r^{\prime}\right) \rho_{x}\left(r^{\prime}\right) d r^{\prime}
\end{aligned}
$$

Substituting the current density expansion in the vector term of the above equation, we have,

$$
\boldsymbol{E}_{\boldsymbol{x}}^{\boldsymbol{e}}(\boldsymbol{r})=\frac{j \omega}{b} \sum_{n=1}^{N-1} I_{x n} \int_{s_{n}} \vec{G}_{A}^{x x}\left(r \mid r^{\prime}\right) T_{x}\left(r-r_{n}\right) d r^{\prime}+\frac{d}{d x} \int_{s} G_{V}\left(r \mid r^{\prime}\right) \rho_{x}\left(r^{\prime}\right) d r^{\prime}
$$

In the first term of the MPIE equation, $s_{n}$ is the area of the associated current cell and $r_{n}$ is the center of the cell. Similarly, substitution into the second (scalar) term will give,

$$
\begin{gathered}
\boldsymbol{E}_{\boldsymbol{x}}^{\boldsymbol{e}}(\boldsymbol{r})=\frac{j \omega}{b} \sum_{n=1}^{N-1} I_{x n} \int_{s_{n}} \vec{G}_{A}^{x x}\left(r \mid r^{\prime}\right) T_{x}\left(r-r_{n}\right) d r^{\prime} \\
+\frac{1}{j \omega a b} \sum_{n=1}^{N-1} I_{x n} \frac{\partial}{\partial x} \int_{s_{o n}} G_{V}\left(r \mid r^{\prime}\right)\left(R\left(r-r_{n}^{+}\right)-R\left(r-r_{n}^{-}\right)\right) d r^{\prime}
\end{gathered}
$$

In the above equation, $s_{o n}$ is the area of individual charge cell. and $r_{n}^{ \pm}$are the centers of associated charge cells which make up one current cell. To simplify the analysis, a vector potential $\Gamma_{A}$, which is created by the surface current and a scalar potential $\Gamma_{V}$, created from a distribution of surface charge, is introduced. Therefore, 


$$
\Gamma_{A}^{x x}\left(r \mid r_{n}^{\prime}\right)=\int_{s_{n}} \vec{G}_{A}^{x x}\left(r \mid r^{\prime}\right) T_{x}\left(r^{\prime}-r_{n}\right) d r^{\prime}
$$

and

$$
\Gamma_{V}\left(r \mid r_{o n}\right)=\int_{s_{o n}} G_{V}\left(r \mid r^{\prime}\right) R\left(r^{\prime}-r_{o n}\right) d r^{\prime}
$$

In general calculations, the above equations must be evaluated by using a numerical integration method. But in cases where the source and observation points are located within the same cell, numerical integration is not possible due to singularity. Thus, to avoid problems, the Green's function should be separated into their regular and singular parts. The singular parts can be integrated analytically and then included back into the main formulation. The analytic solutions are very crucial and will be further discussed in this chapter.

A simplified version of MPIE can now be written as,

$$
\boldsymbol{E}_{\boldsymbol{x}}^{\boldsymbol{e}}(\boldsymbol{r})=\frac{j w}{b} \sum_{n=1}^{N-1} I_{x n} \Gamma_{A}^{x x}\left(r \mid r_{n}{ }^{\prime}\right)+\frac{1}{j \omega a b} \sum_{n=1}^{N-1} I_{x n} \frac{\partial}{\partial x}\left[\Gamma_{V}\left(r \mid r_{n}{ }^{+}\right)-\Gamma_{V}\left(r \mid r_{n}{ }^{-}\right)\right]
$$

A suitable test function is needed for the application of MPIE into MOM. The test function must be compatible with the basis functions and as suggested in studies by Mosig and Gardiol [3], unidimensional rectangular pulses are used. The pulse functions are suitable to the basis functions that were chosen and also it can contribute considerably in the efficiency of the algorithm. Each of these functions are non-overlapping and located between the centers of two adjacent charge cells, which form a single current cell. 


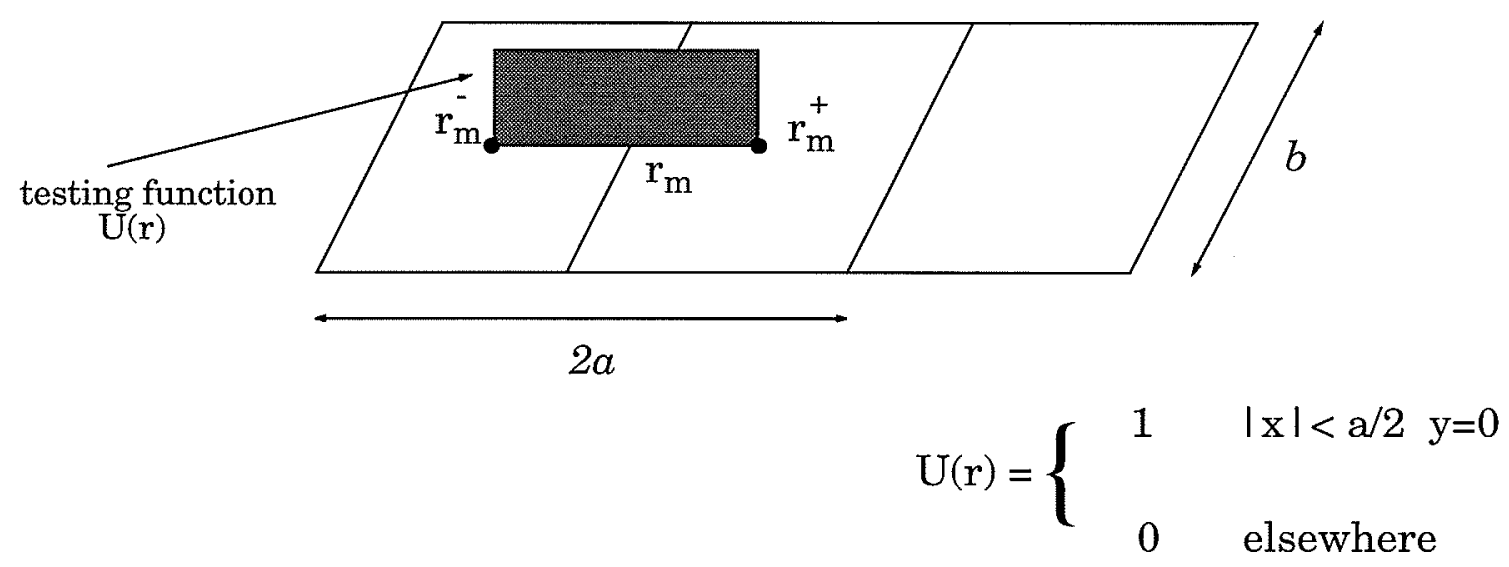

Figure 3.4 : Testing functions (1-dimensional rectangular pulse function)

The set of the testing function can be written as,

$$
\sum_{m=1}^{N-1} U_{m}\left(r-r_{m}\right)
$$

In the above equation, $r_{m}$ is the center of the associated current cell, as shown in Fig. 3.4. The testing function is applied through the formation of an inner product for the MPIE given in equation 3.20. Therefore forming the inner product of the testing function with the MPIE, we obtain :

$$
\left\langle U_{m}\left(r-r_{m}\right), \boldsymbol{E}_{\boldsymbol{x}}^{\boldsymbol{e}}(\boldsymbol{r})\right\rangle=\int_{r_{m}^{-}}^{r_{m}^{+}} \boldsymbol{E}_{\boldsymbol{x}}^{\boldsymbol{e}}(\boldsymbol{r}) d x=V_{x}^{e}
$$

From the first term of eqn. 3.20 , we have

$$
\frac{j \omega}{b} \sum_{n=1}^{N-1} I_{x n} \int_{r_{m}^{-}}^{r_{m}^{+}} \Gamma_{A}\left(r \mid r_{n}{ }^{\prime}\right) d x
$$

Similarly, for the second term, 


$$
\frac{1}{j \omega a b} \sum_{n=1}^{N-1} I_{x n} \int_{r_{m}^{-}}^{r_{m}^{+}} \frac{\partial}{\partial x}\left[\Gamma_{V}\left(r \mid r_{n}^{+}\right)-\Gamma_{V}\left(r \mid r_{n}^{-}\right)\right] d x
$$

Further simplification of the second term can be achieved by removing the derivative by the application of the inner product

$$
\begin{gathered}
\frac{1}{j \omega a b} \sum_{n=1}^{N-I} I_{x n}\left[\Gamma_{V}\left(r_{m}^{+} \mid r_{n}^{+}\right)-\Gamma_{V}\left(r_{m}^{+} \mid r_{n}^{-}\right)\right]- \\
I_{x n}\left[\Gamma_{V}\left(r_{m}^{-} \mid r_{n}^{+}\right)-\Gamma_{V}\left(r_{m}^{-} \mid r_{n}^{-}\right)\right]
\end{gathered}
$$

There are $(N-l)$ number of current cells and also the same number of testing functions. This will yield an $(N-1) \times(N-1)$ system of linear equations and it can be expressed in terms of two dimensional matrix in order to simplify the formulation and numerical evaluation. The system of linear equation is now transformed into a simple electric current/impedance relationship, where the RHS of the MPIE equation is the product of current and impedance.

$$
\left[V_{x m}\right]=\left[I_{x n}\right]\left[Z_{m n}\right]
$$

With a proper specification of the source column, i.e. $\left[V_{x m}\right]$, we can solve for the unknown current distribution. Further derivation of the impedance matrix setup is provided in the following section.

\subsection{Moment Matrix Setup and Numerical Considerations}

In the previous section, the MPIE has been completely transformed into a system of linear equations. These linear equations are derived in terms of a square 


\section{Source Location}

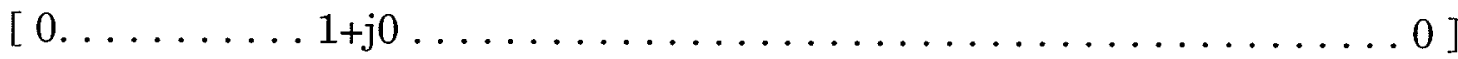

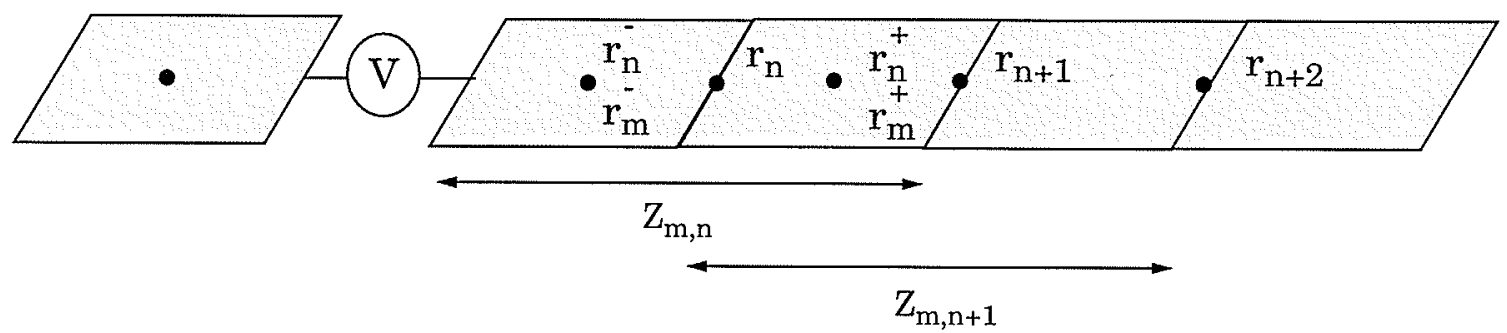

Figure 3.5 : Delta-gap excitation for $x$-directed current cells

From the impedance matrix expression, equation 3.28 , we can see that each individual term in the matrix involves multiple integrations for both vector and scalar potentials. A Gaussian quadrature technique is implemented for the actual integration algorithm.

When the observation point is located within the source cell or very close to it, i.e. the diagonal terms, application of Gaussian quadrature technique will not provide reasonably accurate solutions for both the vector and scalar potential terms. Furthermore, singularities for Green's functions exist if the source and observation points are located within the same cell. This leads to situations where the solution may not converge properly. Thus, for diagonal terms, the dominant term in both Green's functions is given by the static Green's functions, which are

$$
\begin{gathered}
G_{\text {Astatic }}^{x x}=\frac{\mu_{o}}{4 \pi} \frac{1}{\left|r-r^{\prime}\right|} \\
G_{\text {Vstatic }}=\frac{1}{2 \pi \varepsilon_{o}\left(\varepsilon_{r}+1\right)} \frac{1}{\left|r-r^{\prime}\right|}
\end{gathered}
$$

From the above approximate equation, it can be seen that the vector potential of the Green's function is completely independent of substrate parameters whereas 
the scalar term depends on the effective permittivity of the substrate. The vector and scalar component of the impedance matrix can now be expanded in terms of its' corresponding static terms as [2]

$$
\begin{aligned}
& \Gamma_{A}=\int\left(G_{A}^{x x}-G_{\text {Astatic }}^{x x}\right) T_{x} d s+\int G_{\text {Astatic }}^{x x} T_{x} d s \\
& \Gamma_{V}=\int\left(G_{V}-G_{\text {Vstatic }}\right) R_{x} d s+\int G_{V \text { static }} R_{x} d s
\end{aligned}
$$

The first term for both scalar and vector expressions can be integrated normally by implementing a Gaussian quadrature (GQ) technique. But the second term for both expressions needs to be integrated analytically and its full derivation is provided in Appendix A. The analytic integration was studied previously by Yeung [2] and was found to be quite satisfactory in terms of numerical efficiency. A full numerical procedure is outlined in [4].

Going back to the Gaussian quadrature technique : it basically involves choosing a number of quadrature points for integration and for accurate results an odd number of points should be used. The reason being that by choosing odd number of points the center of the associated current cell will be included in the integration process. In our case, its applied for integration situations where the source and observation points are not located within the same cell. The use of odd number of points leads to a higher probability of source and observation points coinciding, when located within the same cell. Therefore, odd number of qudrature points are taken only for non-horizontal terms in the matrix and even number of quadrature points are taken for the horizontal terms, where the occurrence of singularity is inevitable. The number of quadrature points depends on the cell size as well as the location of the source cell from the observation cell. 
The convergence of the solution is achieved easily when the observation cell is located very far from the source cell. This also depends on cell sizes and the smaller the cell size the higher the probability of fast convergence. Also, as the distance between source and observation point gets bigger the convergence in the solution of vector and scalar terms is easier. This can be clarified by observing the Green's function itself, as shown in Fig. 3.6. It shows the real and imaginary component of vector potential Green's function. We can see that the Green's function becomes slowly oscillating function with large distance and has less variation in the solution. Thus, the calculation procedure at large separation is less exhaustive. However, we can see that as the distance between the source and observation points become smaller, the Green's function value becomes larger. Therefore, for these situations, higher number of quadrature points should be used to obtain accurate results.
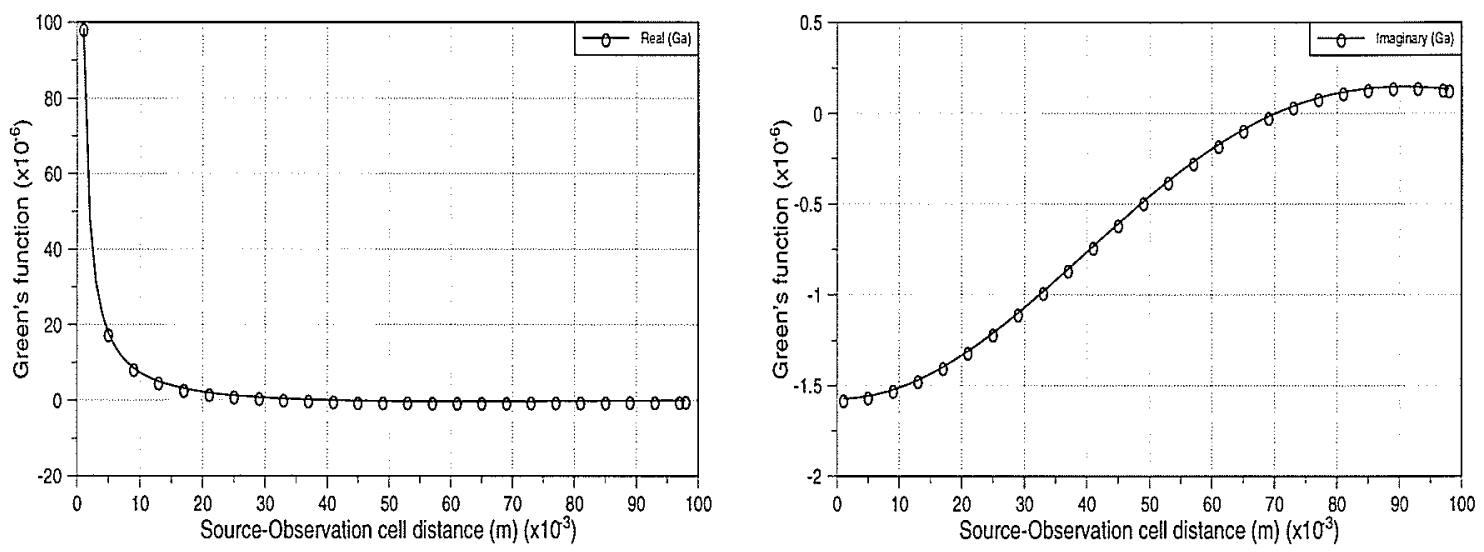

Figure 3.6 : Real and Imaginary component of Vector Potential Green's function

In our case, for non-diagonal terms only five points were implemented for Gaussian Quadrature integration method and for diagonal terms the number of points was increased to ten. This significantly contributes to the efficiency of the final calculation. 
Some numerical considerations will be discussed in the next section. First, we have to take into account the $y$-directed currents for the solution. To make the calculation more efficient, some programming techniques will be proposed in the concluding section.

\subsubsection{Y-Directed Currents}

The concept and expressions derived in the previous sections can be implemented for the solution of y-directed currents. To avoid redundancy, we will not discuss the whole procedure, but the final derivations are provided in Appendix B. In the final solution of MPIE/MOM, both $\mathrm{x}$ - and $\mathrm{y}$ - directed currents must be considered. For illustration purposes, a rectangular strip of length $l$ and width $w$ is considered, as shown in Fig. 3.7.

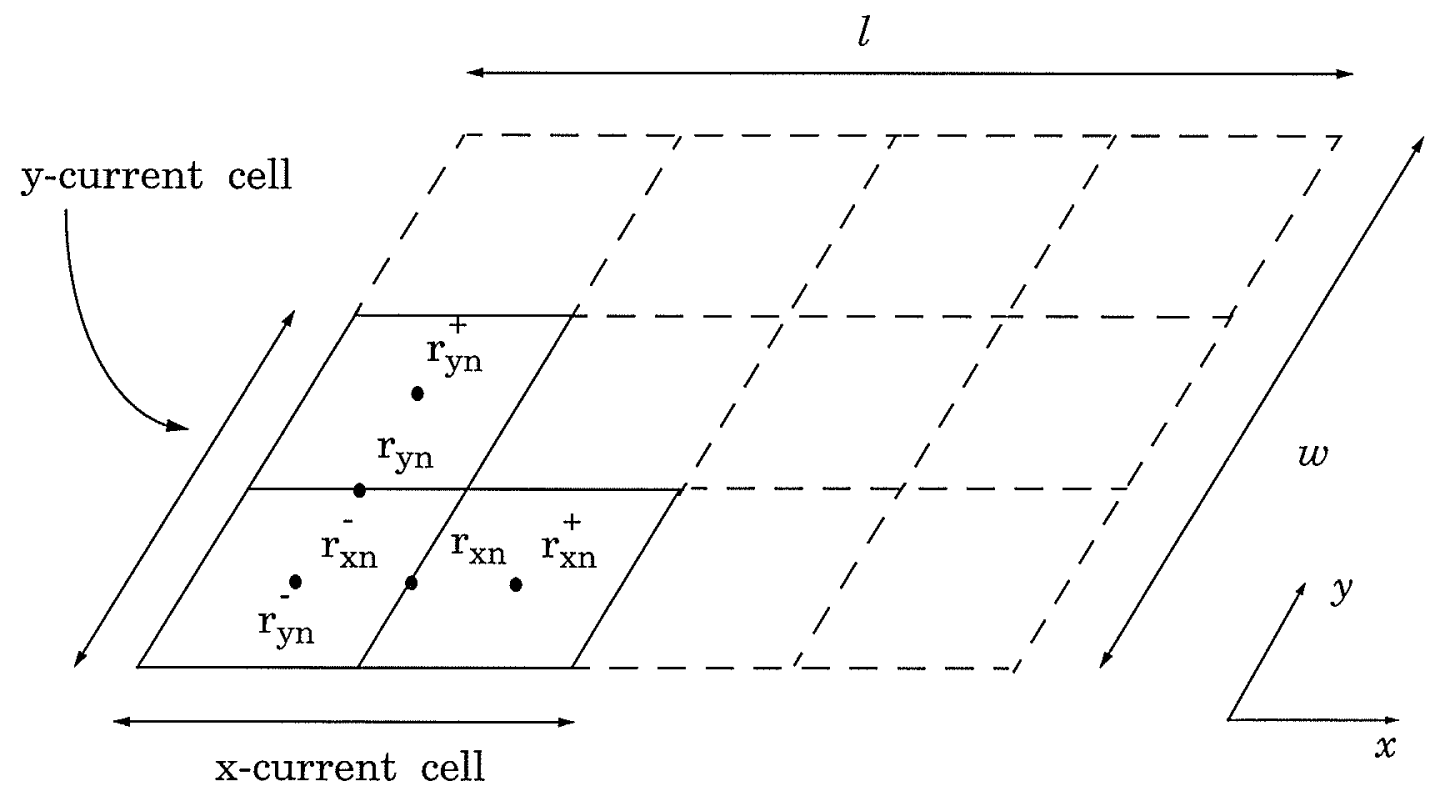

Figure $3.7: x$ - and $y$-directed current cells for a microstrip conductor

For the above conductor having $M \times N$ charge cells, there will be $(M-1) N$ current cells along x-direction and $M(N-1)$ along y-direction. Every y-directed current 
cells support $2 \mathrm{D}$ rooftop functions, as in the $\mathrm{x}$-directed cells. The same is true for the testing functions. The expressions and matrix elements can be easily obtained by carefully interchanging the couple terms $(x, y),(a, b)$ and $(M, N)$ within the defined expressions. Important expressions for $\mathrm{y}$-directed current cells are provided in Appendix B. Thus, the final matrix equation to solve for the unknown currents along $\mathrm{x}$ - as well as $\mathrm{y}$-direction is given by,

$$
\left[\begin{array}{ll}
\mathrm{Z}_{(M-I) N \times(M-I) N}^{x x} & \mathrm{Z}_{M(N-I) \times(M-I) N}^{x y} \\
\mathrm{Z}_{(M-I) N \times M(N-I)}^{y x} & \mathrm{Z}_{M(N-I) \times M(N-I)}^{y y}
\end{array}\right]\left[\begin{array}{c}
I_{x} \\
I_{y}
\end{array}\right]=\left[\begin{array}{c}
V_{x}^{e} \\
V_{y}^{e}
\end{array}\right]
$$

In the above matrix equation, $Z^{x y}=Z^{y x}$ and consists of

$$
\begin{aligned}
Z_{m n}^{x y}= & \frac{1}{j \omega a b} \sum_{n=1}^{N-1}\left[\Gamma_{V}\left(r_{m}^{+} \mid r_{n}^{+}\right)-\Gamma_{V}\left(r_{m}^{+} \mid r_{n}^{-}\right)\right]- \\
& {\left[\Gamma_{V}\left(r_{m}^{-} \mid r_{n}^{+}\right)-\Gamma_{V}\left(r_{m}^{-} \mid r_{n}^{-}\right)\right] }
\end{aligned}
$$

The total number of matrix elements is $[(M-1) N \times M(N-1)]^{2}$. This turns out to be a very big matrix and requires an exhaustive computation. Obviously, it will also depend on the size of the structure and number of cells within the structure. Larger cells will definitely increase the accuracy of the solution but will result in an increase in computation time as well as data storage capacity. To balance trade-offs between the two situation, there should a compromise.

From the above matrix equations, we have seen that the current distribution along both $\mathrm{x}$ - and $\mathrm{y}$ - directions can be obtained from the MPIE/MOM solution. In some applications the calculation of y-directed currents is not very important. For example in the analysis of simple microstrip lines and feed lines with considerably small widths, only the x-directed currents are sufficient to obtain necessary design 
characteristics. However, the solution of y-directed current is equally important in most other type of structures. In the calculation of far field radiation pattern, the contribution of currents along both direction is critical. In this work, to simplify the MPIE solution as well as to budget run time, the y-directed currents are computed only when desired. This does not however jeopardize the accuracy of the solution in any way. Some considerations regarding the computation of the MPIE/MOM solution will be discussed in the next section. This will involve introduction of numerical techniques to save CPU time.

\subsubsection{Programming Considerations}

It is recommended that equal dimension cell sizes be used for the analysis of general microstrip structures. It is more convenient to use a large number of same sized cells rather than fewer number of cells of varying dimensions. However, this can not be achieved sometimes due to the irregularity in the conductor shapes. Also, this may account for extra computation time due to the increase in the number of cells to accommodate the cell size equality. Some numerical techniques can be implemented to counter these problems.

Since the matrix is more dominant in diagonal terms rather than off-diagonal terms, some approximations may be used for non-diagonal terms. Previous study by Mosig and Gardiol [3] shows that the numerical integration associated with the scalar and vector potential of the Green's function may be replaced by analytical approximation for a large source to observation separation. Therefore, the vector and scalar potential terms of the MPIE, given in equations 3.18 and 3.19 , can be written as, 


$$
\begin{aligned}
& \Gamma_{A}^{x x}\left(r \mid r_{n}^{\prime}\right)=\frac{1}{\mu_{o}} \vec{G}_{A}^{x x}\left(r \mid r^{\prime}\right) T_{x}\left(r^{\prime}-r_{n}\right)\left(k_{o} a b\right) \\
& \Gamma_{V}\left(r \mid r_{o n}\right)=\varepsilon_{o} G_{V}\left(r \mid r^{\prime}\right) R\left(r^{\prime}-r_{o n}\right)\left(k_{o} a b\right)
\end{aligned}
$$

For this work, the above approximation is implemented only for specified nondiagonal terms and it is varied for different applications. The main reason is that we have already introduced approximation to the Green's function and by implementing further approximation to the calculation we might end up with an error dominated solution. But for a general case, if the distance is more than 20 times greater than the length of the associated cell, the analytical approximation is implemented. As it will be shown in the examples later, the accuracy is not affected significantly. This is also true if we have to analyze large structures. If very large number of cell sizes are needed, it's numerically efficient to use the above approximation method for big source to observation point separations.

The above described method drastically cuts down the run time and requires less intensive computation. Since we are already implementing approximations for the Green's function itself, it may not be very wise to use any more numerical approximation in the computation. However, some methods that can reduce computation time is applied in our solution. One approach is to use the "comparison" technique. Since the calculation of Green's function depends on the absolute distances between the source and observation point, we can avoid some repetion in the calculation by comparison, i.e. $Z_{m n}=Z_{n m}$. This is very critical in reducing run time for the calculation procedure and is implemented in the calculation of all general microstrip structures. One important requirement for this technique is that the cell sizes must be of equal dimensions. It cannot be used for the analysis of 
structures where the use of different cell sizes is required.

Consider a strip having $n$ number of segments, as shown in Fig. 3.8. The line is segmented into $\mathrm{n}$ cells along the length and there are no segments along $\mathrm{y}$ direction. The cells have equal dimension and support $\mathrm{x}$-directed currents only.

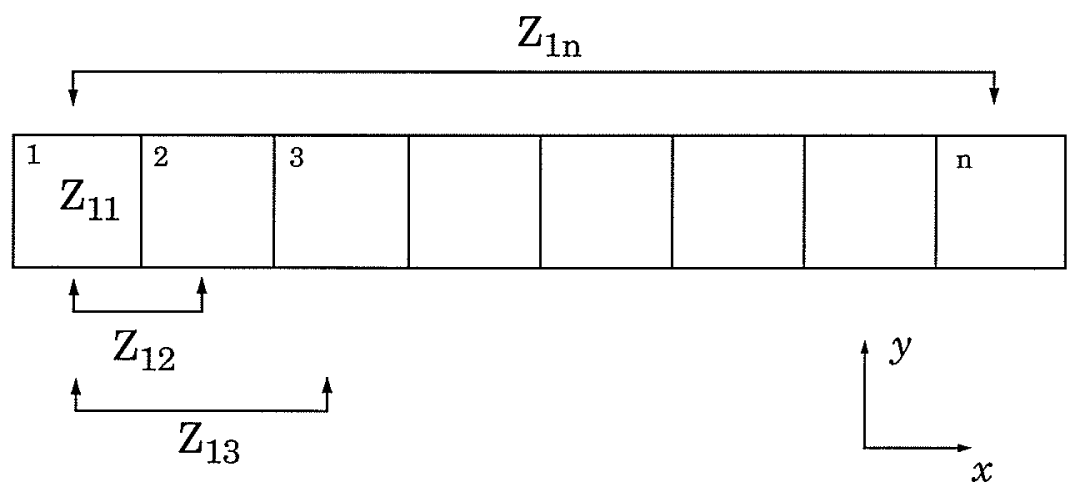

Figure. 3.8 : Impedance calculation procedure for microstrip line

In the above conductor strip, the first calculation of $Z$ values $\left[Z_{11}, Z_{12}, Z_{13}, Z_{14} \ldots Z_{1 n}\right]$ will yield the impedance values for all the current cells, by the method of comparison. Thus, calculation of $Z_{21}, Z_{31} \ldots Z_{n 1}$ is not required and this will considerably reduce the run time. Only a single calculation of impedance values will produce the solution for all the elements. This is crucial when analyzing large microstrip structures such as antennas integrated with feed structures and will be implemented in the calculation of microstrip antennas.

The Green's function based solution has been completely derived in this chapter. Also, several numerical considerations and techniques have been discussed. The following chapter focuses on the procedure for acquiring frequency dependent design characteristics such as the S-paramters and far field radiation patterns. 


\section{Chapter IV}

\section{S-Parameters and}

\section{Far-Field Radiation Patterns}

\subsection{Introduction}

This chapter focuses mainly on the two significant characteristics associated with the design of microstrip antennas and circuits : S-parameters and far field radiation patterns. In antenna design, S-Parameter terms such as the reflection coefficient and transmission coefficient predicts the performance of the antenna to a certain degree. The basic theory associated with the extraction of frequency dependent S-Parameters is explained in this chapter. The E- and $\mathrm{H}$ - plane far field radiation patterns for microstrip structures and antennas will also be discussed. 


\subsection{Frequency Dependent S-Parameters}

The S-parameters can be extracted directly from the MPIE/MOM solution. A single port microstrip antenna is considered as an example to illustrate the extraction of frequency-dependent S-parameters. However, the same technique can be applied to all general microstrip structures. The technique involves the calculation of S-parameter terms, such as the reflection coefficient, by considering the maximum and minimum values of the current distribution along the transmission line. A microstrip line with Delta-gap generator excitation is implemented as the feeding mechanism. The radiating patch is fed by a microstrip line as shown in Fig. 4.1.

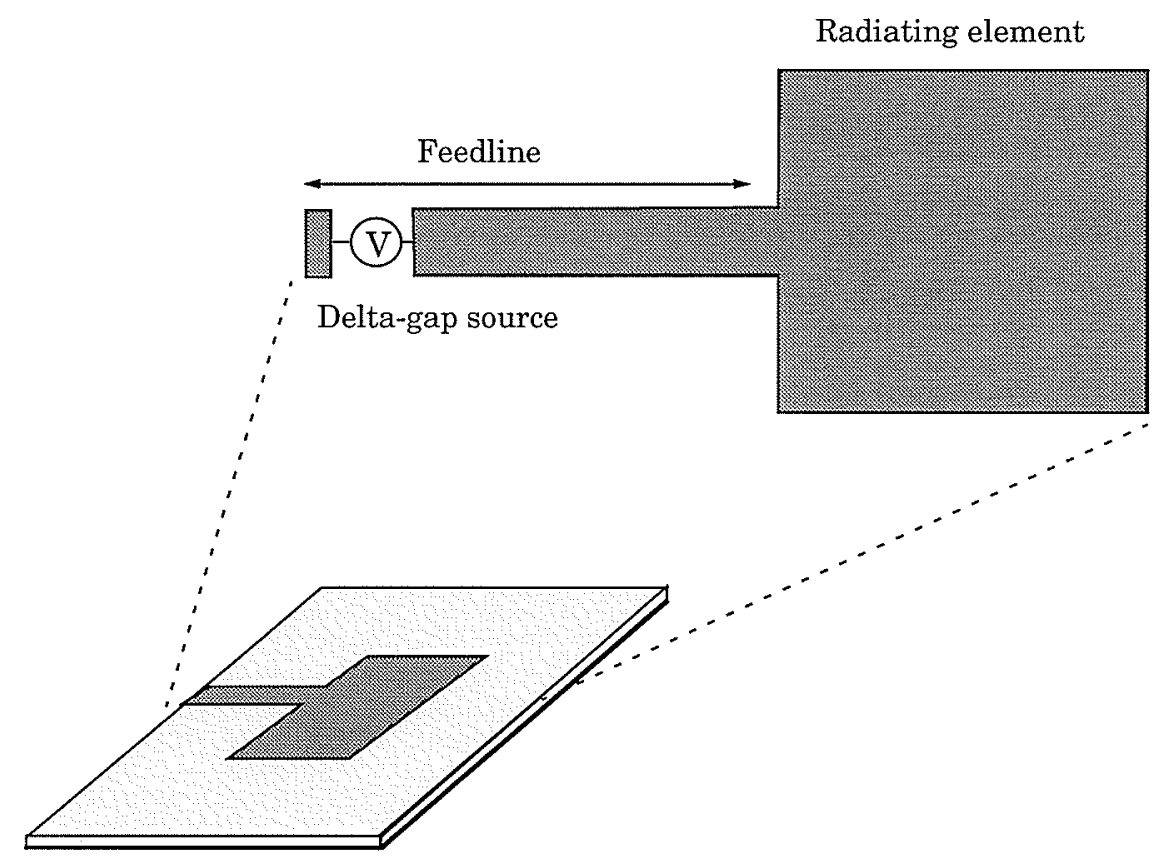

Figure 4.1 : Simulation setup for a single feed microstrip antenna

The feedline should be considerably large to avoid higher order modes generated by the discontinuity between the feedline and the radiating element. There will be a region in the transmission line where the higher order modes are suppressed. In this region, only the quasi-TEM mode propagates. For the analysis , 
only the longitudinal current component is required. This means that the computation of only $\mathrm{x}$-directed currents is sufficient for the whole section of the feedline. A standing wave pattern can be obtained for the current distribution along the quasi-TEM line. Assuming that no radiation occurs in the feedline, we can implement transmission line theory for the analysis of standing wave pattern to obtain the reflection and transmission coefficients. A transmission line model of the above antenna system is given in Fig. 4.2.

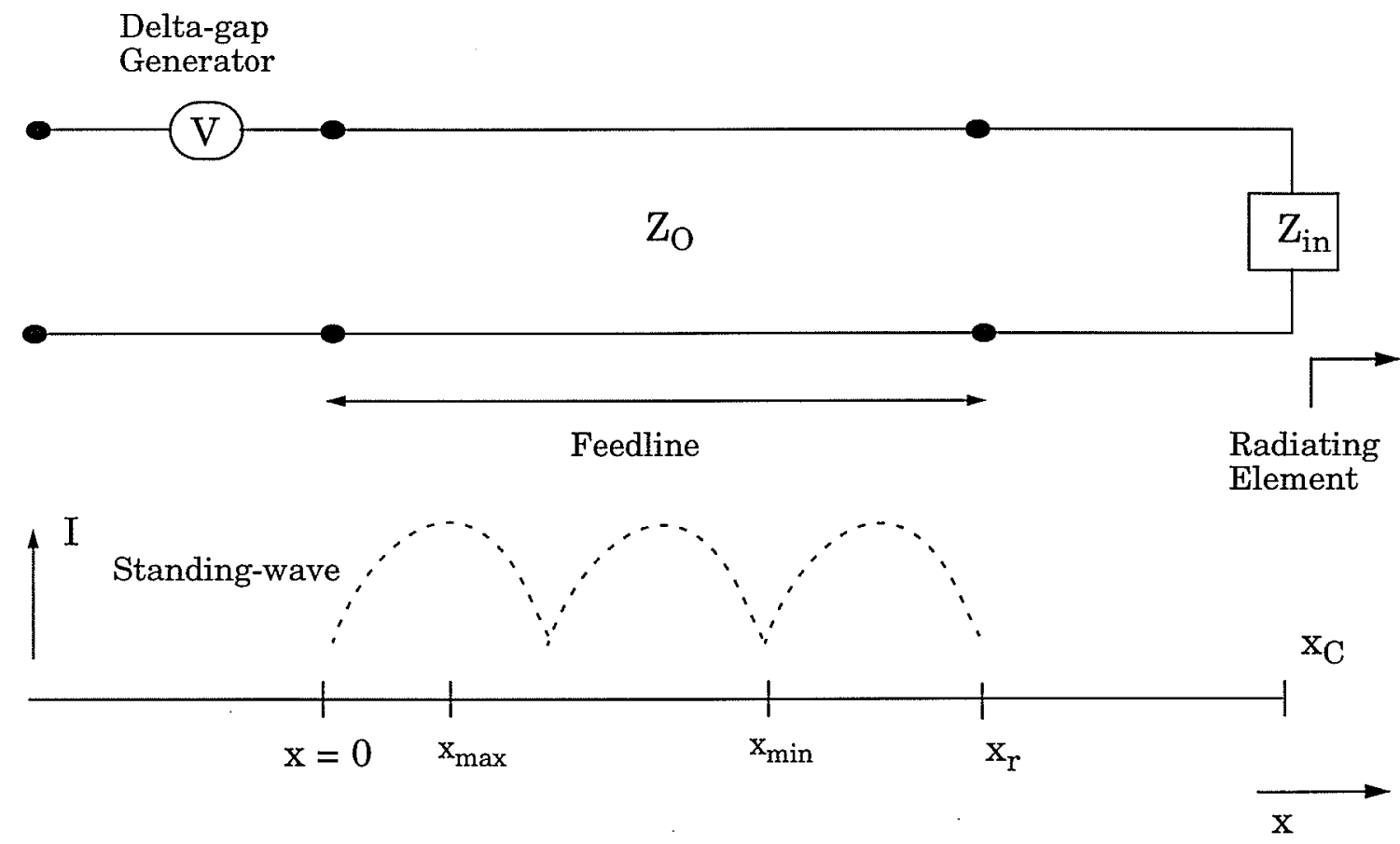

Figure 4.2 : Transmission line model for single feed microstrip antenna

In the model, a reference point $x_{r}$ is located after the voltage source and before the radiating patch. Furthermore, the distance between $x_{r}$ and $x_{o}$ is considerably large to obtain a quasi-TEM behaviour between the voltage source and the reference point $x_{r}$. The analysis concept is mainly based upon the values of current maxima and minima at this portion of the feedline [2]. The current 
distribution along the feedline can be obtained from the MPIE/MOM solution. Then location of these maxima and minima can be determined by simple observation of the standing wave pattern. But for accurate results, an interpolation method should be implemented. In our study a cubic spline interpolation method is used to determine the exact location of current maxima and minima.

At the reference plane $x_{r}$, the reflection coefficient $\Gamma$ is obtained from,

$$
\begin{gathered}
\Gamma_{r}=|\Gamma| e^{j \theta} \\
=\frac{S W R-1}{S W R+1} e^{j \theta}
\end{gathered}
$$

where, $\theta=\frac{4 \pi d}{\lambda_{g}}$ and $d=\left(x_{r}-x_{\min }\right)$. In the above notation $x_{\text {min }}$ is the location where the first current minima occurs. The guided wavelength of the feedline is obtained from transmission line theory [19] and is given as,

$$
\lambda_{g}=2 \times\left|x_{\text {max }}-x_{\min }\right|
$$

The standing wave ratio of the pattern can be calculated in terms of current values by,

$$
S W R=\frac{\left|I_{\max }\right|}{\left|I_{\min }\right|}
$$

For the characterization of a single port circuit, such as the patch antenna, analysis of the reflection coefficient is sufficient to validate the match between the feeding mechanism and the radiating element. Therefore, the magnitude and phase of the reflection coefficient is given by [15],

$$
\left|\Gamma_{i n}\right|=\frac{\left|I_{\max }-I_{\min }\right|}{\left|I_{\max }+I_{\min }\right|}
$$




$$
\theta_{\text {phase }}=\frac{2 \pi}{\lambda}\left(x_{r}-x_{\max }\right)
$$

The return loss, which is defined as the ratio in decibels of the incident power to the reflected power, is given as

$$
R_{L}=20 \log \frac{1}{\left|\Gamma_{i n}\right|}
$$

Similarly, the transmission loss can also be obtained. Transmission loss is defined as the ratio of incident power and the transmitted power. As in the case of return loss, the expression is obtained in decibels.

$$
T_{L}=20 \log \frac{1}{1-\left|\Gamma_{i n}\right|^{2}}
$$

The return loss is one of the significant design characteristics of microstrip structures, including antennas. The main reason is that it helps to determine the impedance matching between source and the circuit. An accurate calculation of the return loss is very important in order to predict the final performance of the antenna.

\subsection{E- and H- Plane Radiation Patterns}

A horizontal electric dipole is used for the general derivation of the far field pattern of microstrip structures. In the second chapter, it has been shown that the electric field can be expressed in terms of vector and scalar potentials. From equation

$$
\boldsymbol{E}=-j \omega \boldsymbol{A}-\nabla V
$$

The current density $\boldsymbol{J}_{\boldsymbol{s}}$ can be obtained from an equation derived in the previous chapter. Therefore, going back to the electric field-current relationship, we have [3] 


$$
\boldsymbol{E}=\int_{s} G_{E} \cdot \boldsymbol{J}_{s}\left(r^{\prime}\right) d s^{\prime}
$$

where, $G_{E}$ represents the electric dyadic Green's function and is given by

$$
G_{E}=-j \omega G_{A}\left(r \mid r^{\prime}\right)+\frac{1}{j \omega \varepsilon \mu} \nabla\left(\nabla G_{V}\left(r \mid r^{\prime}\right)\right)
$$

Since we are concerned with the far field radiation pattern, we can assume that the distance between the source and observation point is infinitely large. This will allow the application of asymptotic expressions for the Green's function instead. Therefore, the radiation field is then obtained by transforming the asymptotic terms into spherical coordinates. The field relationships for unit current are [6],

$$
\begin{gathered}
E_{r}=0 \\
E_{\theta}=\frac{\cos \phi \cos \theta \sqrt{\varepsilon_{r}-\sin ^{2} \theta}}{\sqrt{\varepsilon_{r}-\sin ^{2} \theta}-j \varepsilon_{r} \frac{\cos \theta}{\tan \left(k_{o} h \sqrt{\varepsilon_{r}-\sin ^{2} \theta}\right)}} \frac{e^{-j k r}}{r} \\
E_{\phi}=\frac{\sin \phi \cos \theta}{\sqrt{\varepsilon_{r}-\sin ^{2} \theta}} \frac{e^{-j k r}}{r} \\
\cos \theta-j \frac{\tan \left(k_{o} h \sqrt{\varepsilon_{r}-\sin ^{2} \theta}\right)}{r}
\end{gathered}
$$

In order to calculate the far field pattern of the surface conductor, a pattern multiplication method is used. The main consideration here is that the patch or the conductor surface is assumed to be an array of current cells. The current distribution for the entire patch can be obtained by the MPIE/MOM solution. Thus, by introducing pattern multiplication in equation. 4.10, we obtain. 


$$
E_{\theta, \phi}=G_{E}^{x} \sum_{i=1}^{\mathrm{M}-1} \Delta x I_{x i} e^{j k_{o}\left(\grave{\mathrm{x}} \cdot \grave{r}_{i}\right)}+G_{E}^{y} \sum_{i=1}^{N-1} \Delta y I_{y i} e^{j k_{o}\left(\grave{y} \cdot \vec{r}_{j}\right)}
$$

The dyadic Green's function in spherical coordinates is obtained from previous derivation and transforming the remaining electric field expression into spherical coordinates, we can derive the final expression for the radiation field of a microstrip structure. The E-plane and $\mathrm{H}$-plane radiation patterns are calculated for $\phi=0^{\circ}$ and $\phi=90^{\circ}$ respectively [6], [13]

$$
\text { E - Plane }
$$

$$
\begin{gathered}
E_{\theta}=\frac{\sqrt{\varepsilon_{r}-\sin ^{2} \theta} \cos \theta}{\sqrt{\varepsilon_{r}-\sin ^{2} \theta}-j \varepsilon_{r} \frac{\cos \theta}{\tan \left(k_{o} h \sqrt{\varepsilon_{r}-\sin ^{2} \theta}\right)}} \sum_{i=1}^{N-1} \Delta x I_{x i} e^{j k o_{o}{ }^{x}} \\
E_{\phi}=\frac{\cos \theta}{\cos \theta-j \frac{\sqrt{\varepsilon_{r}-\sin ^{2} \theta}}{\tan \left(k_{o} h \sqrt{\varepsilon_{r}-\sin ^{2} \theta}\right)}} \sum_{i=1}^{N-1} \Delta y I_{y i} e^{j k_{o} y_{i}}
\end{gathered}
$$

H - Plane

$$
\begin{gathered}
E_{\theta}=\frac{\sqrt{\varepsilon_{r}-\sin ^{2} \theta} \cos \theta}{\sqrt{\varepsilon_{r}-\sin ^{2} \theta}-j \varepsilon_{r} \frac{\cos \theta}{\tan \left(k h \sqrt{\varepsilon_{r}-\sin ^{2} \theta}\right)}} \sum_{i=1}^{N-1} \Delta y I_{y i} e^{j k_{o} y_{i}} \\
E_{\phi}=\frac{\cos \theta}{\cos \theta-j \frac{\sqrt{\varepsilon_{r}-\sin ^{2} \theta}}{\tan \left(k_{o} h^{\varepsilon_{r}-\sin ^{2} \theta}\right)}} \sum_{i=1}^{N-1} \Delta x I_{x i} e^{j k_{o} x_{i}}
\end{gathered}
$$

In the above equations, the current distributions along $\mathrm{x}$ and $\mathrm{y}$ must be 
carefully implemented. In other words, the current distribution associated with each current cells must be systematically chosen in relation to the location of the cells.

In this chapter, the theory associated with the extraction of S-parameters and calculation of far field radiation patterns have been discussed thoroughly. In the following chapter, the accuracy of the theoretical results, derived in this chapter, will be compared with the existing results as well as results obtained from a software tool. Furthermore, experimental results of a designed microstrip antenna will also be presented 


\title{
Chapter V
}

\author{
Numerical Results
}

\subsection{Introduction}

The MPIE/MOM solution discussed in the previous chapters will now be used to analyze actual microstrip structures. Before this method can be applied to the design of various microstrip structures, it is important that the method be validated first. In this chapter the MPIE/MOM solution generated from our study is verified by comparing it to the results obtained from the research work done by Yeung [2], Couture [4] and Balanis [7]. Also, the MPIE/MOM solution is used to design and analyze microstrip antennas. Main characteristics such as the reflection coefficient and the E- and H-Plane far field patterns is analyzed and compared with the results obtained from the software tool PRELUDE [25]. Design, analysis and experimental results for a circularly polarized microstrip antennas are also included in this chapter. 


\subsection{Microstrip Transmission Lines and Dipoles}

Before implementing the MPIE/MOM formulation for the analysis of complex structures, it is tested for simple microstrip problems with known solutions. The verification involves testing the accuracy as well as the rate of convergence. Three separate test simulations are performed. The first test involves a convergence test for a full-wavelength, center fed horizontal dipole over an infinite ground plane. The second simulation test looks at the accuracy of the input impedance calculation and the last test focuses on the calculation of characteristic impedance for microstrip lines of varying width dimensions. Furthermore, the effect of varying the height of the substrate on the performance of microstrip line is observed. This analysis is based mainly on the input impedance characteristic of the microstrip line.

\subsubsection{Microstrip Dipoles}

Microstrip dipoles have been analyzed by many researchers [8], [11], [16] and is a good choice for validating our solution because of the availability of results from their work. For the first simulation test, we analyze a full-wavelength horizontal microstrip dipole placed in free space over an infinite ground plane. The width of the line is considered to be significantly small in comparison to the length of the microstrip line.

The main assumptions are that the microstrip dipole to be analyzed has zero thickness, perfect-conducting transmission line located horizontally at a vertical distance $h$ over the ground plane. The ground plane is also considered to be a perfectly conducting material. For this case, frequency is taken as a constant, such that $\lambda_{o}=1$. The dipole is fed at the center by a delta-gap voltage source, as shown 
in Fig. 5.1. The dielectric constant is $\varepsilon_{r}=1$ for free space.

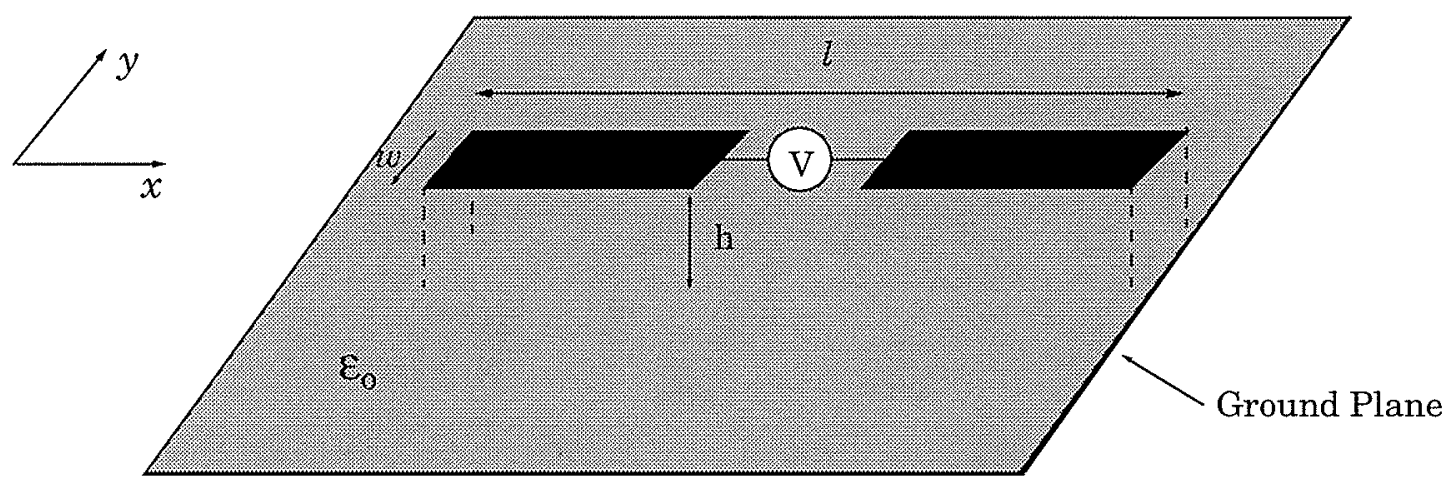

Figure 5.1 : Microstrip dipole with delta-gap excitation

The conductor is segmented only along $\mathrm{x}$-direction, i.e. there are only $\mathrm{x}$ directed current cells. Since the width of the conductor is very small, discretization along $\mathrm{y}$-direction is not necessary. An even number of charge cells are used because that will generate an odd number of current cells, which translates to the location of the voltage source exactly in the middle of the line. In other words, this causes an alignment between the center of the dipole and the middle current cell, which is the location of the voltage source. The length of the strip is $l=1 \lambda_{o}$, the width is $w=0.01 \mathrm{~mm}$ and the height from the dipole to the ground plane is $h=0.1 \lambda_{o}$.

The Current distribution for the dipole is obtained by the MPIE/MOM solution for different number of charge cells. Our main goal here is to test the convergence of the solution in relation to the density of current (or charge) cells along the microstrip surface. A reasonably large number of charge cells are used for the analysis.

The current values are obtained in terms of magnitude and phase and are shown in Fig. 5.2 and Fig. 5.3 respectively. Fig 5.2 shows the magnitude of the 
current distribution along the length of the strip. Each current value is calculated exactly at the center of each current cell. Five simulations are performed for different cell densities.

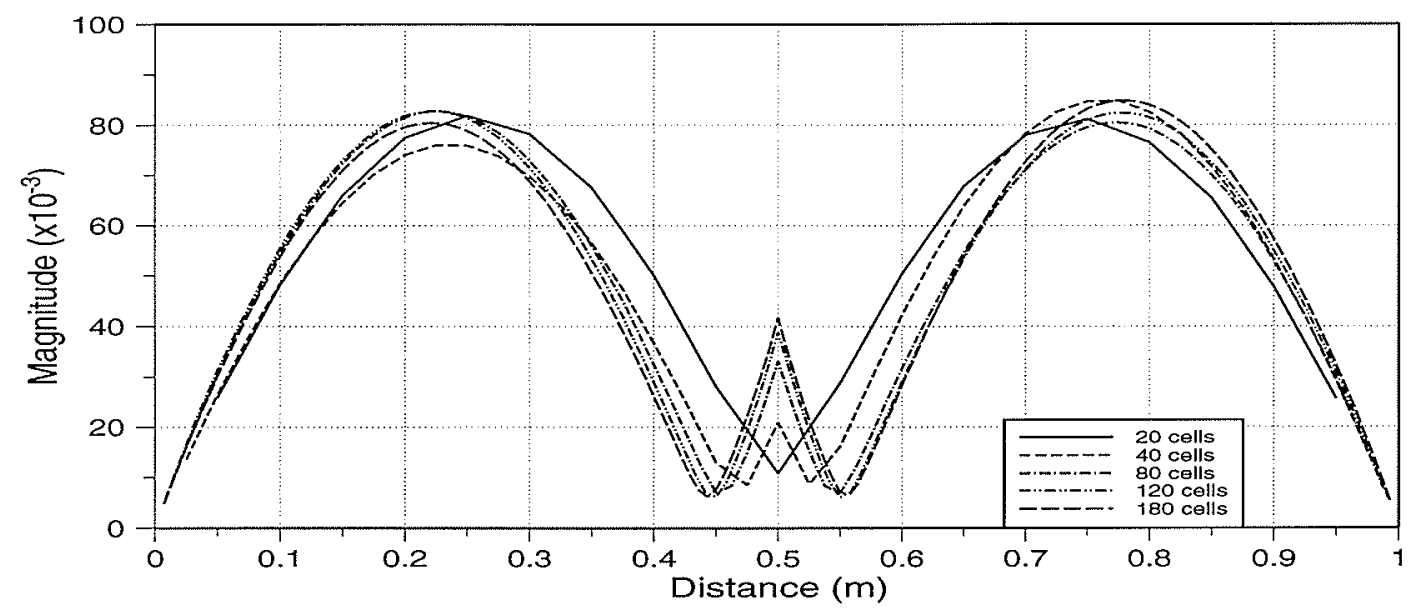

Figure 5.2 : Current magnitude for single wavelength microstrip dipole

From the above figure it can be seen that the solution converges very fast. The standing wave pattern seems to have converged after 40 cells. Fig 5.3 shows the phase distribution for the full-wavelength transmission line.

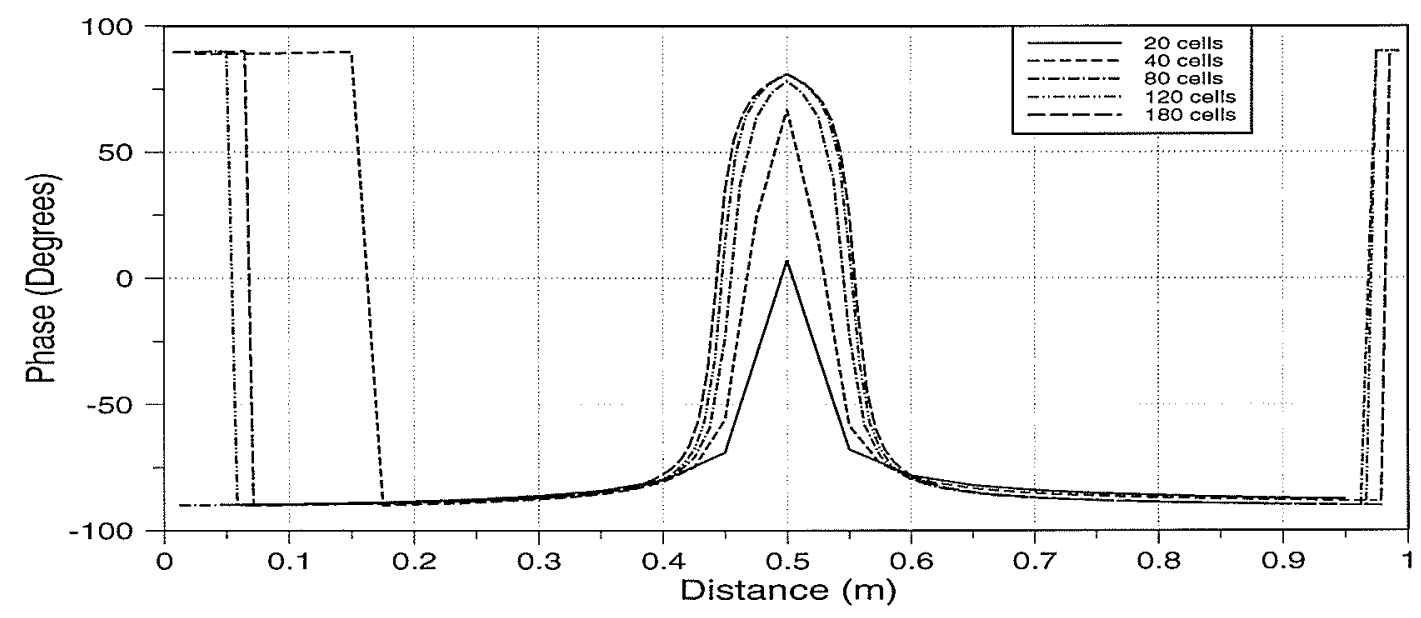

Figure 5.3 : Phase distribution for single wavelength microstrip dipole

For this case no approximation was introduced in the calculation and it did 
not upset the run-time of the simulation. For example, for 120 charge cells, the whole simulation run took a little less than 8 seconds in a SPARC-1 machine. From above plots, it can be seen that the MPIE/MOM formulation is valid when $\varepsilon_{r}=1$ and convergence is achieved quite easily. With the convergence confirmed, we can calculate the input impedance by implementing frequency and comparing the input impedance values with the values obtained from [2].

For the second test, a half wavelength center fed dipole is simulated to analyze the input impedance. The calculation is performed at $f=3 G H z$ and a dielectric constant $\varepsilon_{r}=1$ (free space) is used. The length of the dipole at the given frequency is $l=0.05 \mathrm{~m}$ and $w=0.01 \mathrm{~mm}$ chosen as the width of the dipole, in order to compare it to the existing results [4]. The input impedance for the dipole is calculated for various ground plane to dipole height $h$. For a total of 100 charge cells, the input impedance value is calculated from $h=1 \mathrm{~mm}$ to $h=10 \mathrm{~mm}$ and is shown in Fig. 5.4. Also, the impedance values obtained from the [2] is plotted in the same graph.

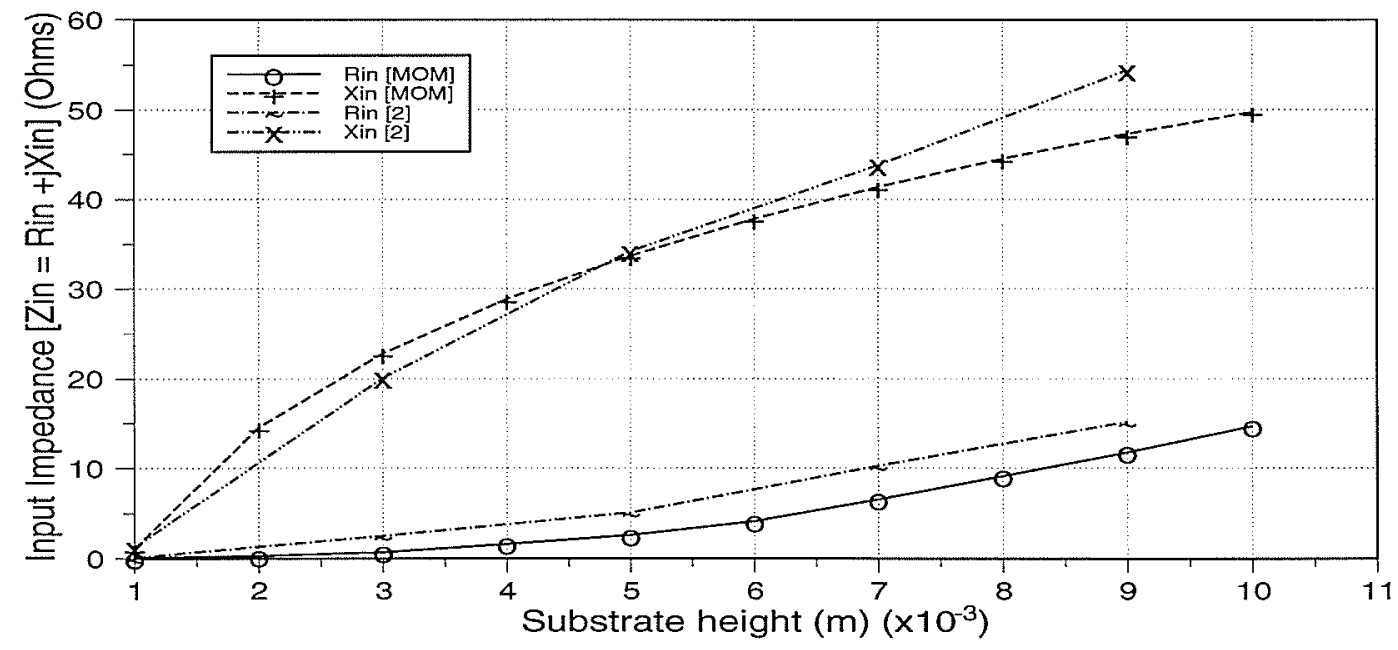

Figure 5.4 : Input impedance of the microstrip line for different ground to dipole height 
A slight discrepancy can be seen in our simulation results. For these type of analysis, the use of further numerical approximations should be avoided. In Fig. 5.5, once again the input impedance values are calculated for a wide range of ground plane to dipole heights.

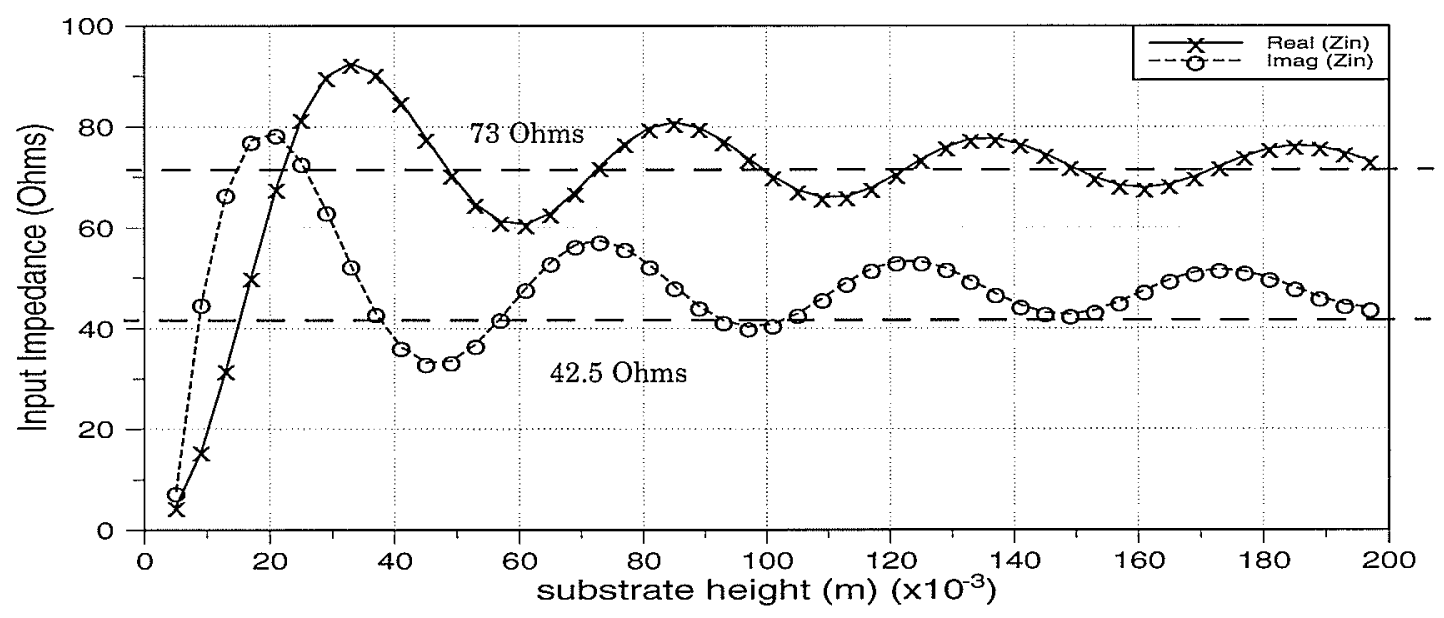

Figure 5.5 : Input impedance vs. ground plane to dipole height

In the above graph, we can see that the input impedance value approaches a virtually constant value as the height between the dipole and the ground plane approaches a very large value. The real part of the impedance approaches $73 \Omega$ and the imaginary part shifts close to $42.5 \Omega$, which are the exact known values of a halfwavelength dipole and was obtained from Balanis [7]. These values also agree with the data obtained from similar test performed by Yeung [2]. Thus, from the above data, we can say that the result obtained from the MPIE/MOM solution for a small substrate height is satisfactory and the error can be considered marginal. However, precautions should be taken when analyzing structures having a small substrate height. In other words, further numerical approximations should be avoided.

The impedance values obtained from our MPIE/MOM solution also seem to be 
relatively accurate for thicker substrates. Comparison of the results show a marginal error in our MPIE/MOM solution as opposed to the solution obtained from Sommerfeld Integral equation. The associated errors are present mainly due to the introduction of approximate Green's function. Additional simulation tests are carried out to further explore the accuracy and error domination in the final solution.

\subsubsection{Characteristic Impedance of Microstrip Lines}

In this section, an open microstrip transmission line is analyzed to verify design parameters such as the characteristic impedance $Z_{o}$ and effective relative permittivity $\varepsilon_{\text {eff }}$. The results are compared with the data obtained from a previous work by Couture [4].

First consideration to be made here is that the guided wavelength value is approximately equal to the free space wavelength, so that the source can be located at a distance $0.20 \lambda_{o}$ from one end of the microstrip line. The total length is $l=0.10 \mathrm{~m}$ and the width $w$ is varied according to the simulation tests in [4] for comparison purposes.

The thickness of the substrate is $h=1 \mathrm{~mm}$ and the dielectric constant is $\varepsilon_{r}=2.3$. As mentioned in the previous chapter, the guided wavelength can be found by method of current maxima and minima, which are obtained by implementing the cubic spline method. A typical standing wave pattern for the microstrip line is shown in Fig. 5.6. 


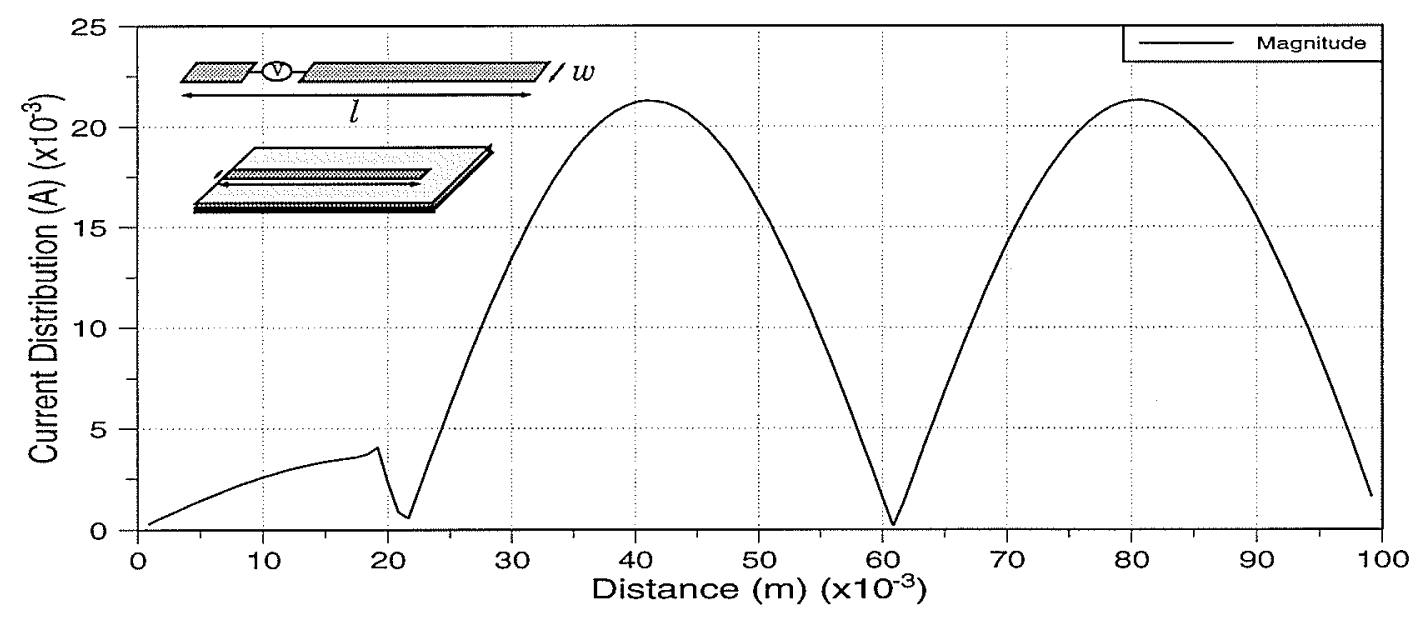

Figure 5.6 : Current distribution for off-center fed microstrip line

In the above standing wave pattern, the total number of charge cells used is 120 and the width is $1 \mathrm{~mm}$. One curious observation is that the location of the source is reflected clearly in the standing wave pattern. A slight peak is observed at about $20 \mathrm{~mm}$, where the source is located. Far-field radiation pattern for both $\mathrm{E}$ - and $\mathrm{H}$ planes are provided in Fig. 5.7. The values are individually normalized to their respective maxmima.

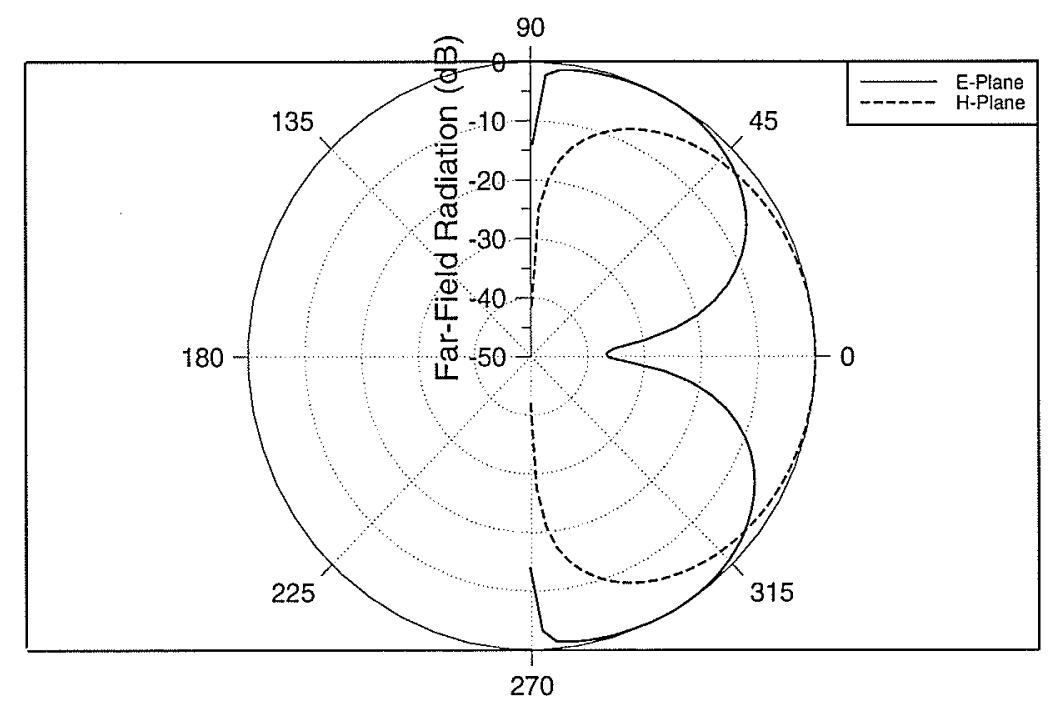

Figure 5.7 : Far field radiation pattern for off-center fed microstrip line 
The guided wavelength of the transmission line can be calculated in terms of the free space wavelength and effective dielectric constant.

$$
\lambda_{g}=\frac{\lambda_{o}}{\sqrt{\varepsilon_{e f f}}}
$$

Since we can calculate the guided wavelength from the current values, the effective dielectric constant and characteristic impedance can be calculated from the following transmission line relationships [18],

$$
\begin{gathered}
\varepsilon_{\text {eff }}=\left(\frac{\lambda_{o}}{\lambda_{g}}\right)^{2} \\
Z_{o}=\frac{1}{\sqrt{\varepsilon_{\text {eff }}}} 60 \ln \left[\frac{6 h}{w}+\sqrt{1+\left(\frac{2 h}{w}\right)^{2}}\right]
\end{gathered}
$$

In this part of the simulation, 130 charge cells are used at a frequency $f=2 G H z$. The result seems to converge around this number of cells but it is slightly higher than the number of cells used in reference [2]. This does not cause any significant change in the final result. Table 1 shows a side by side comparison of the characteristic impedance and effective dielectric constant values obtained from our MPIE/MOM solution and the ones obtained from [4].

Table 5.1 : Characteristic Impedance of Off-Center Fed Microstrip Line

\begin{tabular}{|c|c|c|c|c|}
\hline Width(mm) & Zo (MOM) & Zo (Ref.[4]) & $\varepsilon_{\text {eff }}$ (MOM) & $\varepsilon_{\text {eff }}$ (Ref.[4]) \\
\hline 0.4 & 140.32 & 134.5 & 1.716 & 1.802 \\
\hline 1.0 & 102.12 & 93.5 & 1.751 & 1.841 \\
\hline 2.0 & 68.91 & 64.5 & 1.810 & 1.908 \\
\hline 4.0 & 42.72 & 41.0 & 1.886 & 1.994 \\
\hline
\end{tabular}

From the data table, it can be seen that the results obtained from our MPIE/ MOM solution are very close to the results obtained from Sommerfeld integrals. As expected, slight error is noticable in our calculation because of Green's function 
approximation. However, it does not significantly influence the final result.

In this MPIE/MOM solution, numerical approximation is first introduced in the MOM procedure itself. As mentioned previously, the exact solution is obtained only by applying an infinite number of matrix terms. However this is not possible and thus a reasonable number of matrix elements have to be used. This approximation reduces the accuracy to a certain degree. Since this is unavoidable, further approximation in most MPIE/MOM solutions is prevented through the use of Sommerfeld integrals to calculate Green's function. In our case, since the use of Sommerfeld integrals is totally absent and instead approximate Green's functions are implemented, we are definitely introducing more approximations in the calculation. For example, the data obtained from the simulations show a marginal error associated with our solution. The discrepancy in the results seems to be fairly minimal and does not significantly affect the overall analysis procedure. In the calculation of characteristic impedance of the microstrip lines, we can see that on average the error does not exceed to more than few percents. The worst case has an error of about $9 \%$ for impedance and about $5 \%$ for effective dielectric constant.

\subsection{Microstrip Antennas}

From the analysis of simple microstrip structures, we have seen that the results are in a reasonable agreement with the data obtained from the exact Sommerfeld equations' solution. Now, we will further test the MPIE/MOM solution by analyzing microstrip antennas. First, a single feed microstrip antenna is analyzed and then compared with the results obtained from a software tool called PRELUDE. Next, a circularly polarized antenna will be designed and tested experimentally. The 
main design criterias to be considered here is the input impedance and reflection coefficient. Also, the $\mathrm{E}$ - and $\mathrm{H}$ - plane radiation pattern will be calculated and compared with the PRELUDE results.

\subsubsection{Single Feed/Linearly Polarized Microstrip Antenna}

Microstrip patch antennas with a single feed can be used in different applications. However, due to some disadvantages such as low bandwidth, patch arrays are more popular as opposed to a single patch element. The performance of the array depends critically on the individual elements and care must be taken in designing these elements. In this section a single feed patch antenna is analyzed in terms of it's main design characteristics such as the input impedance and return loss. The numerical results are compared with the data obtained from PRELUDE.

The main design parameters used for the calculation are selected so that it can be simulated with both tools. The antenna is designed at a frequency $f=4 G H z$ and the substrate parameters are : dielectric constant $\varepsilon_{r}=2.2$ and height $h=0.79 \mathrm{~mm}$. The radiating patch is of length $l=25 \mathrm{~mm}$ and width $w=40 \mathrm{~mm}$. The dimensions of the patch are taken such that the width is slightly less than the full wavelength in the substrate and the length is about half wavelength long, for the design frequency. For the first test simulation, the patch is fed directly by a $50 \Omega$ line without the use of any impedance matching, as shown in Fig. 5.8. The width of the feedline is about $2.46 \mathrm{~mm}$. The feedline is made considerably long to suppress higher order modes and to generate quasi-TEM mode only. For this case, the length is $12 \mathrm{~mm}$. 

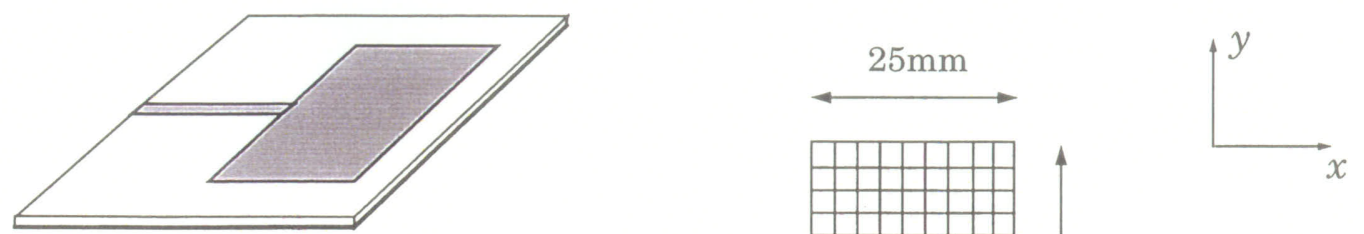

$50 \mathrm{ohm}$ feed line

Figure 5.8 : Segmentation for microstrip antenna

A typical three dimensional standing wave pattern, for an x-directed current, would look similar to the pattern shown in Fig 5.9, which is obtained for 168 current cells at $f=4 \mathrm{GHz}$.
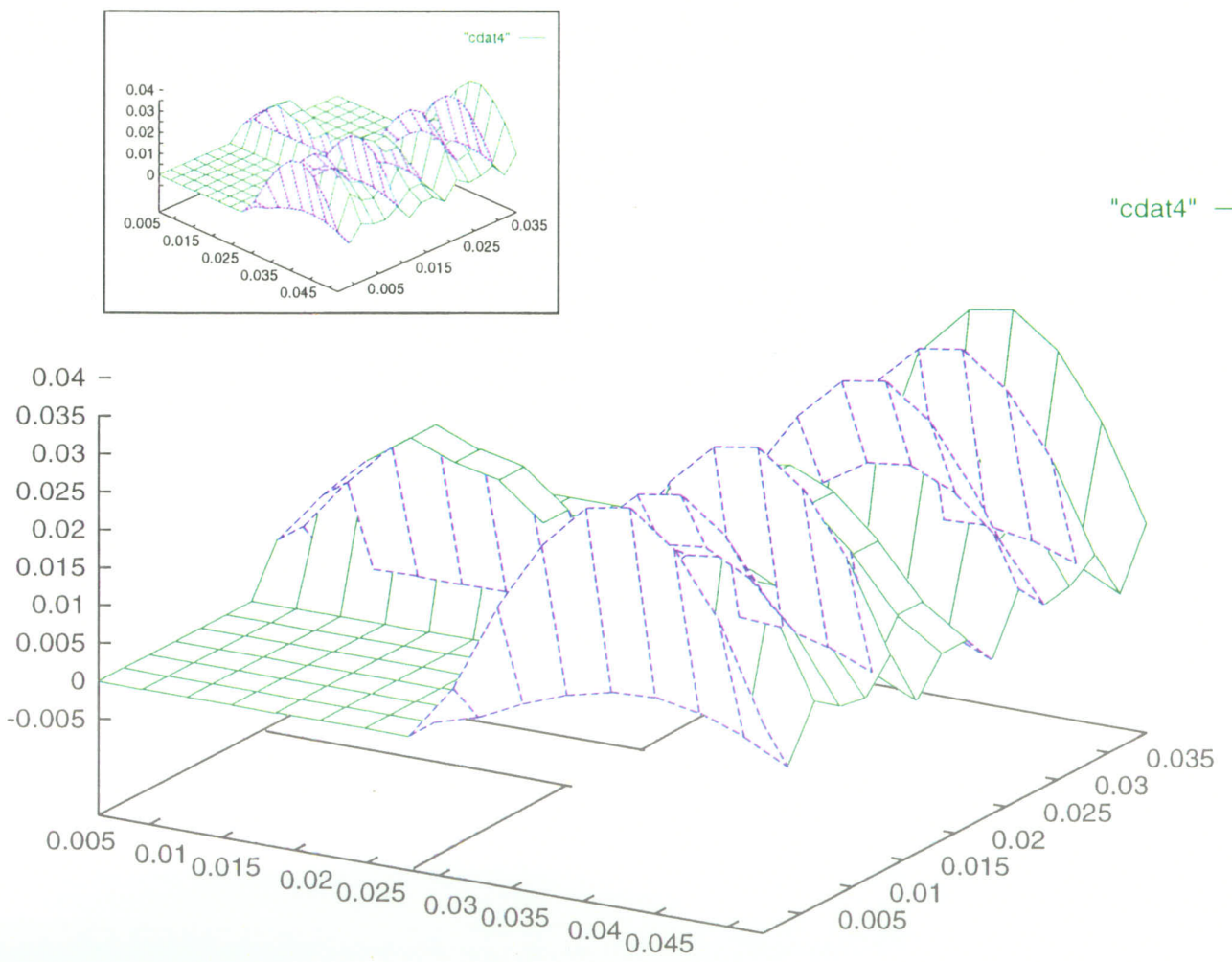

Figure 5.9 : Current distribution along the microstrip antenna 
From the above plot, we can see that the standing wave pattern along the feedline is considerably large due to reflection. Since the antenna is not matched, most of the current will be reflected back and it is clearly noticable in the graph. In the calculation procedure, current distribution are calculated for the whole structure. In large structures this is not necessary, if the structure is symmetric. For example, in the above microstrip antenna, the radiating patch itself can be disected into upper and lower symmetrical segments. Thus, calculation for only one half is needed and solution of the whole patch can be obtained. However, this technique is not implemented in this case to verify the solution, but it will be applied in future simulation examples in order to minimize run time.

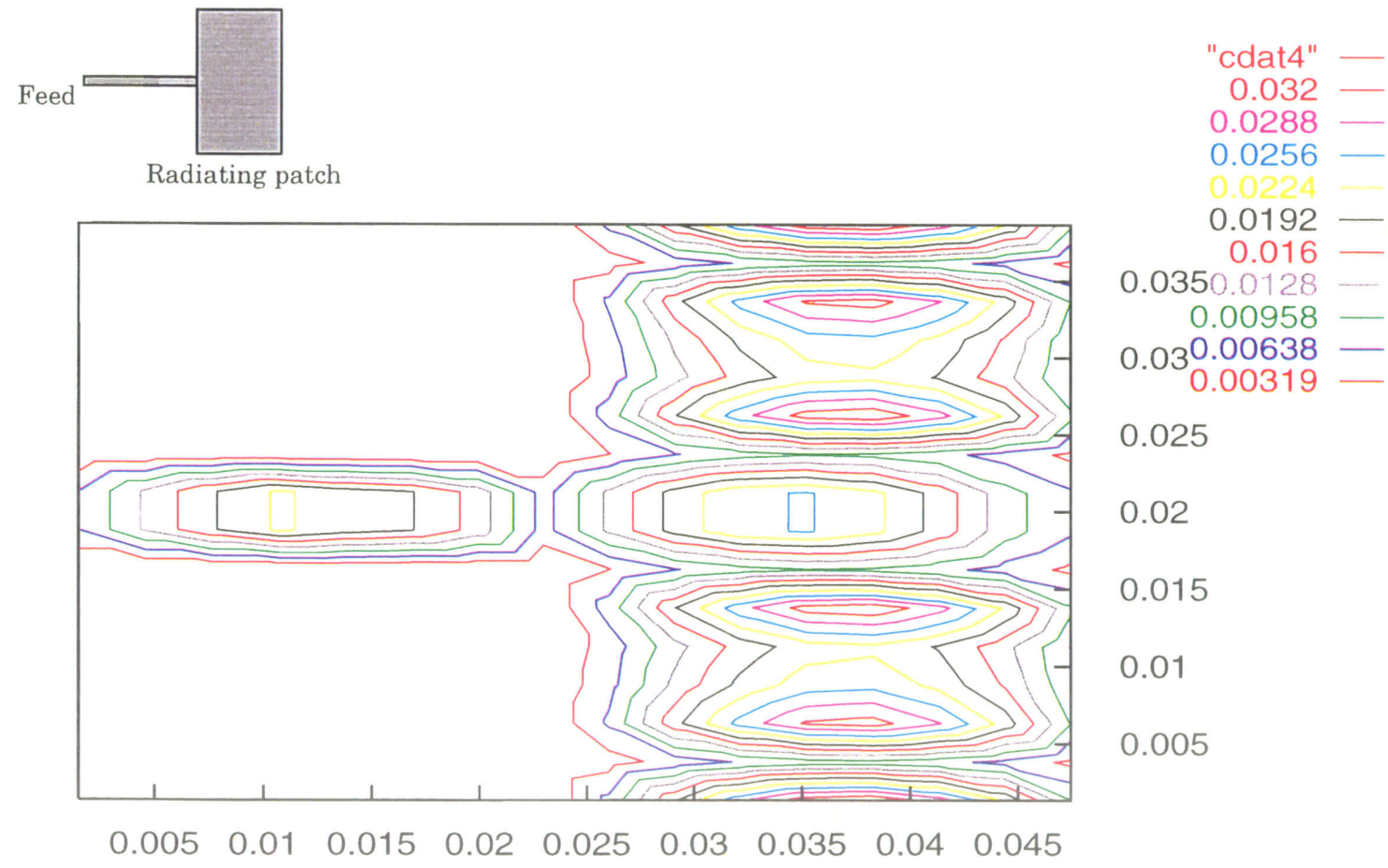

Figure 5.10 : Contour plot for the current distribution along the patch

From the contour plot in Fig. 5.10 we can clearly see that the calculation is qualitatively accurate and current distribution is symmetric for the whole radiating element. This further validates our MPIE/MOM calculation procedure. 
The input impedance values are first calculated and verified by comparing it to the results obtained from PRELUDE. This requires a frequency sweep test and it is performed for frequency range of $f=3.8 \mathrm{GHz}$ to $f=4.2 \mathrm{GHz}$. The input impedance is calculated right on the junction where the feed line is joined by the radiating element. This may accurately predict the input impedance of the radiating element alone. The real component (normalized) of the input impedance values calculated from MPIE/MOM solution is shown in Fig. 5.11. It's normalized to a reference impedance of $50 \Omega$. The resonance frequency lies somewhere between 3.90 $\mathrm{GHz}$ and 3.95GHz. The normalized imaginary component is shown in Fig. 5.12 and on close inspection, we can see that the imaginary part of the input impedance passes through zero at about $3.92 \mathrm{GHz}$. Therefore, at this resonant frequency the input impedance of the patch is approximately $150 \Omega$.

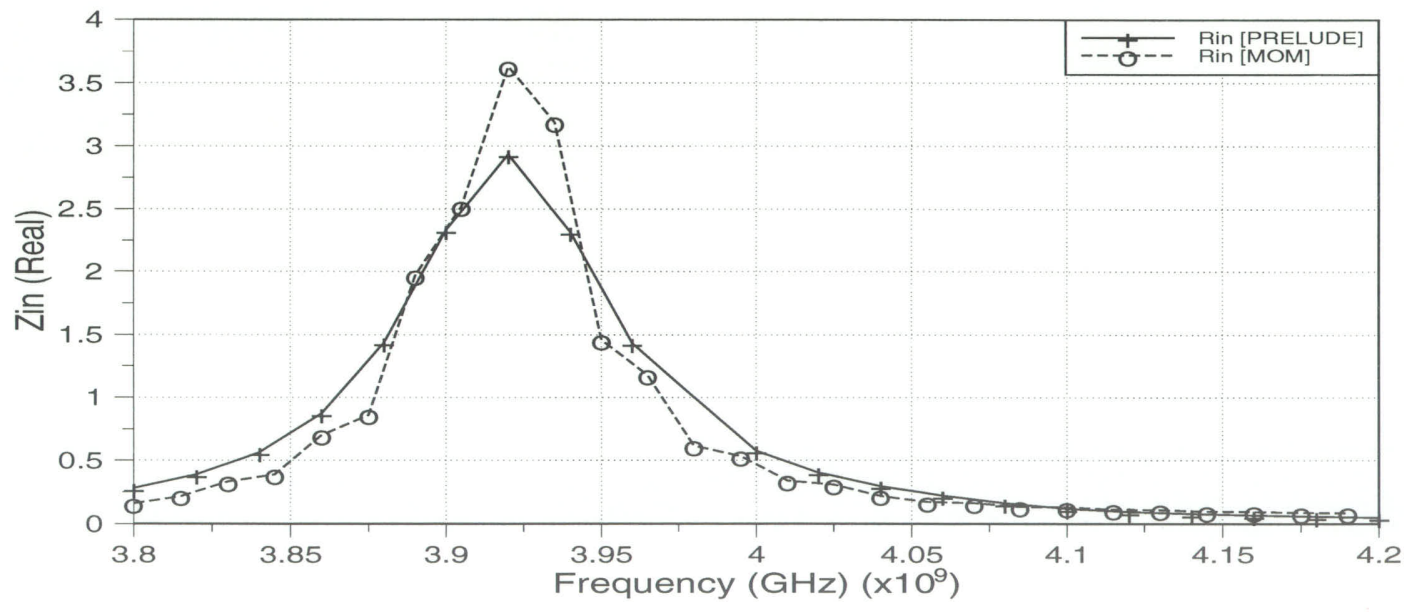

Figure 5.11 : Normalized input impedance (Real) vs. frequency

The same frequency sweep test is performed in PRELUDE and the real and imaginary parts of the input impedance patterns are incorporated in the same graphs, i.e. in Fig. 5.11 and Fig.5.12 respectively. 


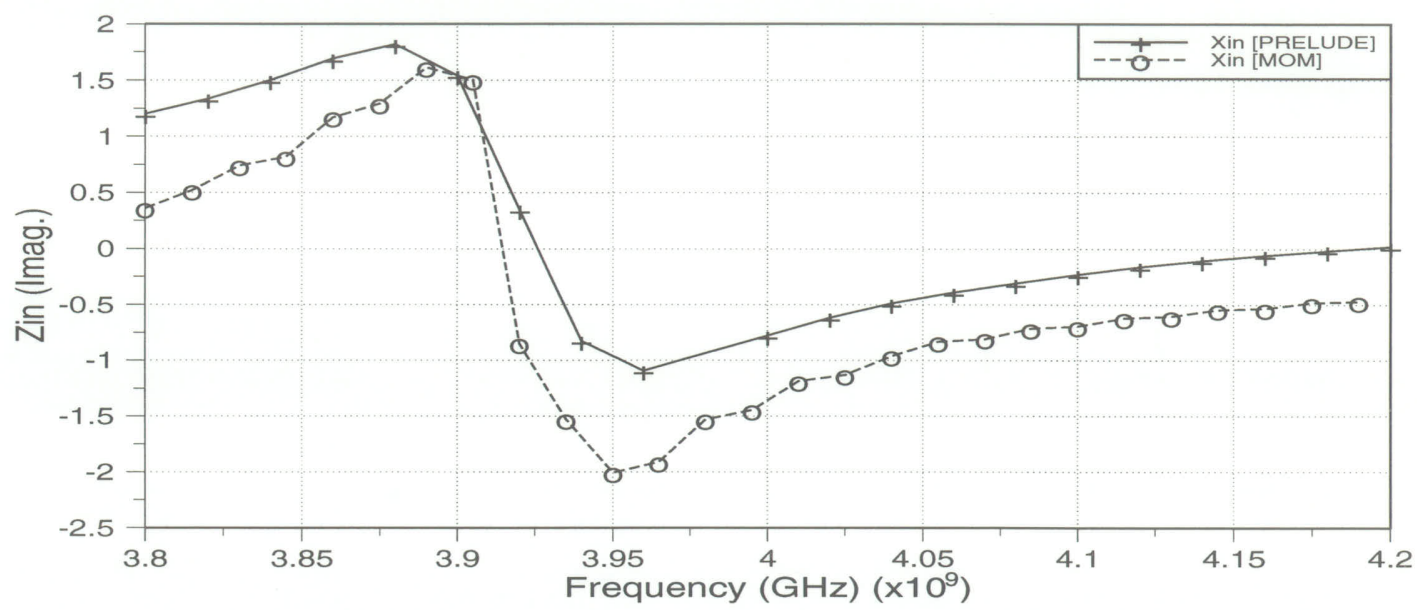

Figure 5.12 : Normlized input impedance (Imaginary) vs. frequency

The impedance values from the above results show that our resonant frequency is about $0.25 \%$ lower than the one obtained from PRELUDE. This is expected due to the introduction of Green's function approximation. Also, we can see an increase in the impedance values around the resonant frequency. As verified in the previous section, an increase in the impedance value is expected from our calculation. However, since the increase is very marginal at resonant frequency, the overall design procedure will not be affected very much. From PRELUDE results, we can observe that the input impedance at resonance is about $144 \Omega$, which is lower than the impedance calculated from MPIE/MOM. Next, the far field radiation patterns will be calculated.

Fig. 5.13 and 5.14 shows the normalized E-plane $\left(\phi=0^{\circ}\right)$ and H-plane $\left(\phi=90^{\circ}\right)$ far field radiation pattern obtained from our calculation and from PRELUDE. 


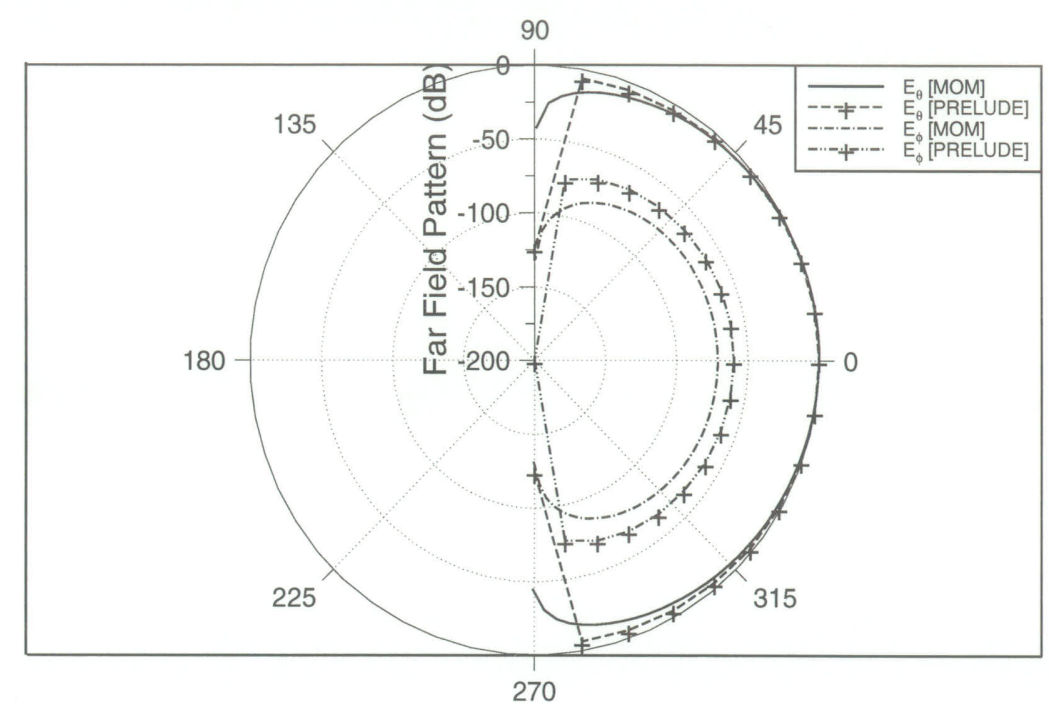

Figure 5.13 : E-Plane radiation pattern for the microstrip antenna

From the E-plane radiation patterns, we can see that the co-polar and crossploar levels from our calculation are almost identical to PRELUDE results. A slight error is noticable but it is not too significant.

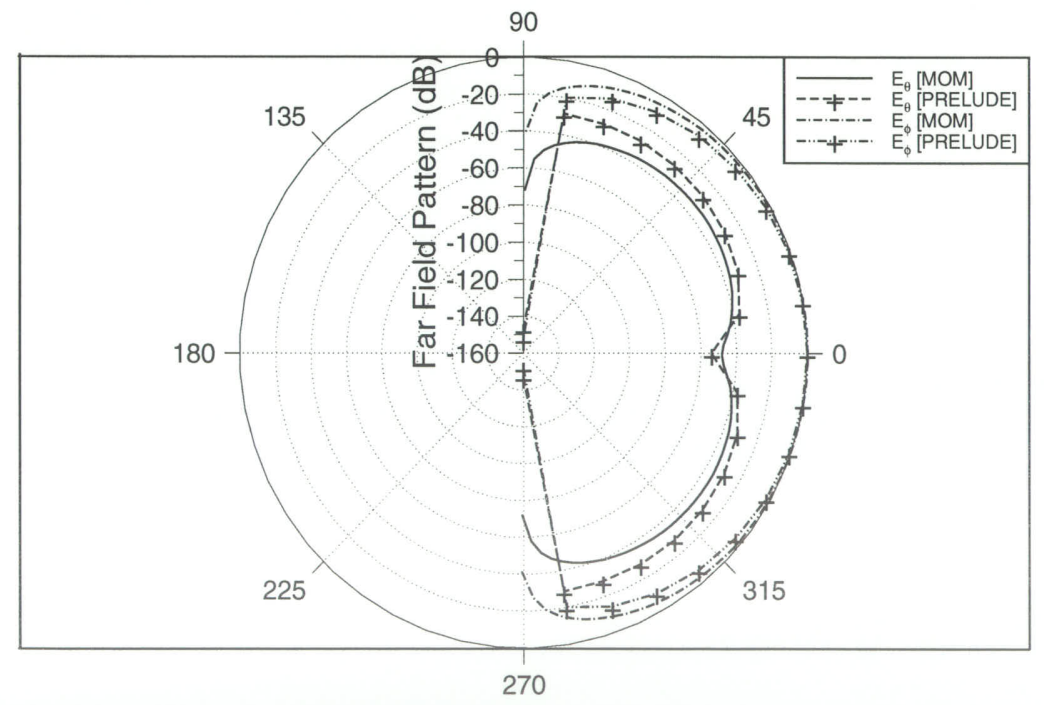

Figure 5.14 : H-Plane radiation pattern for the microstrip antenna

Furthermore, this error is more dominant in the crosspolar level but since the crosspolar values are small and insignificant, there is virtually no concern about the 
error margin in this case. As in the E-plane case, the H-plane radiation pattern results also show a good agreement with the results obtained from PRELUDE with minor error for both copolar and crosspolar values.

Some discrepancies are found in our results when compared with PRELUDE. As the reason stated before, it's caused mainly by the introduction of approximation procedure in the calculation of Green's function. The error we have observed so far does not significantly affect the overall design procedure.

The antenna is matched by adding a simple quarter wave transformer in between the $50 \Omega$ feedline and the radiating patch. To match the antenna, it's resonance frequency must be known. In our case, we will design the matching structure at $3.92 \mathrm{GHz}$. The input impedance of the patch is then the impedance at that frequency multiplied by the reference impedance, which is $50 \Omega$. Since we are considering resonance, the input impedance is assumed to have a pure real value.

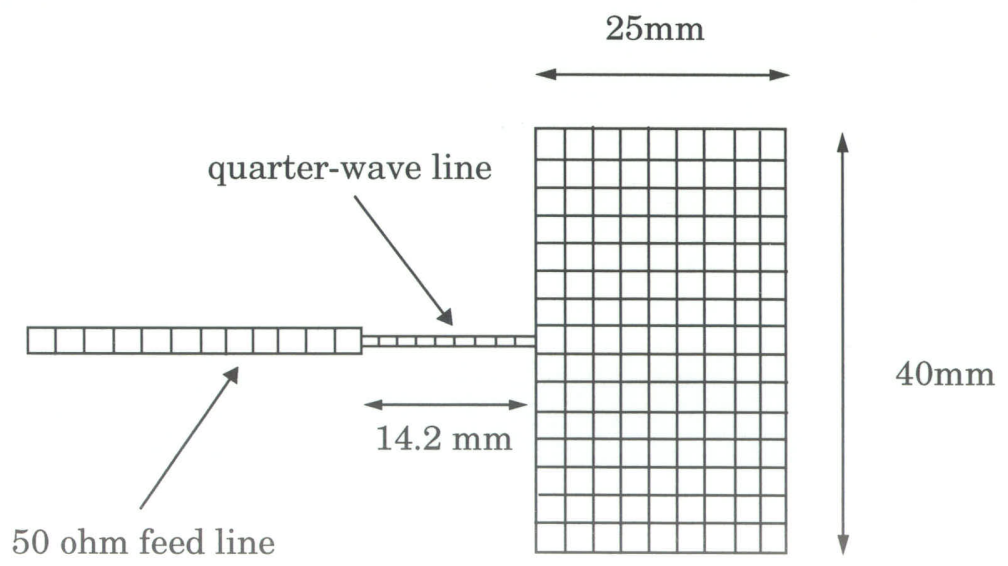

Figure 5.15 : Segmentation for the matched microstrip antenna

We use a simple quarter wave transmission line to match the antenna to the feedline. From transmission-line theory, the characteristic impedance of the quarter- 
wave line can be obtained [19]

$$
Z_{q r t}=\sqrt{Z_{o} Z_{i n}}
$$

where, $Z_{i n}$ is the input impedance of the antenna and $Z_{o}$ is the impedance of the feed line. In our case, the quarter wave line impedance is $87 \Omega$. Therefore, the final dimension of the transformer for the above impedance criteria is : $l=14.2 \mathrm{~mm}$ and $w=0.9 \mathrm{~mm}$. Fig. 5.15 further illustrates our explanation. The results obtained from both simulations follow.

A frequency sweep is performed to verify the matching of the antenna, which is probably the most critical design criteria at this point. Reflection coefficient (S11) for the entire antenna structure is calculated for the same frequency range, i.e. $f=3.8 \mathrm{GHz}$ to $f=4.2 \mathrm{GHz}$. The first run showed that the antenna is slightly off resonance and therefore several test runs are performed for slight variations in the radiator width. It is found that the performance of the antenna is satisfactory when the width of the radiator is changed from $25 \mathrm{~mm}$ to $24.4 \mathrm{~mm}$. Also the width of the quarter-wave line is changed to $0.85 \mathrm{~mm}$. The remaining dimensions are left unchanged.

The reflection coefficient results obtained from PRELUDE and the one obtained from our calculation are shown in Fig. 5.16. For this case, the Dirac-delta voltage source is placed right at the end of $50 \Omega$ feedline. Also, PRELUDE allows the location of source at the end. The location of the source point will definitely influence the outcome of the final solution. For comparison purpose, such as in our case, the source location in both methods should be similar. Therefore, here we have tried to place the source at exactly the same point for both cases. 


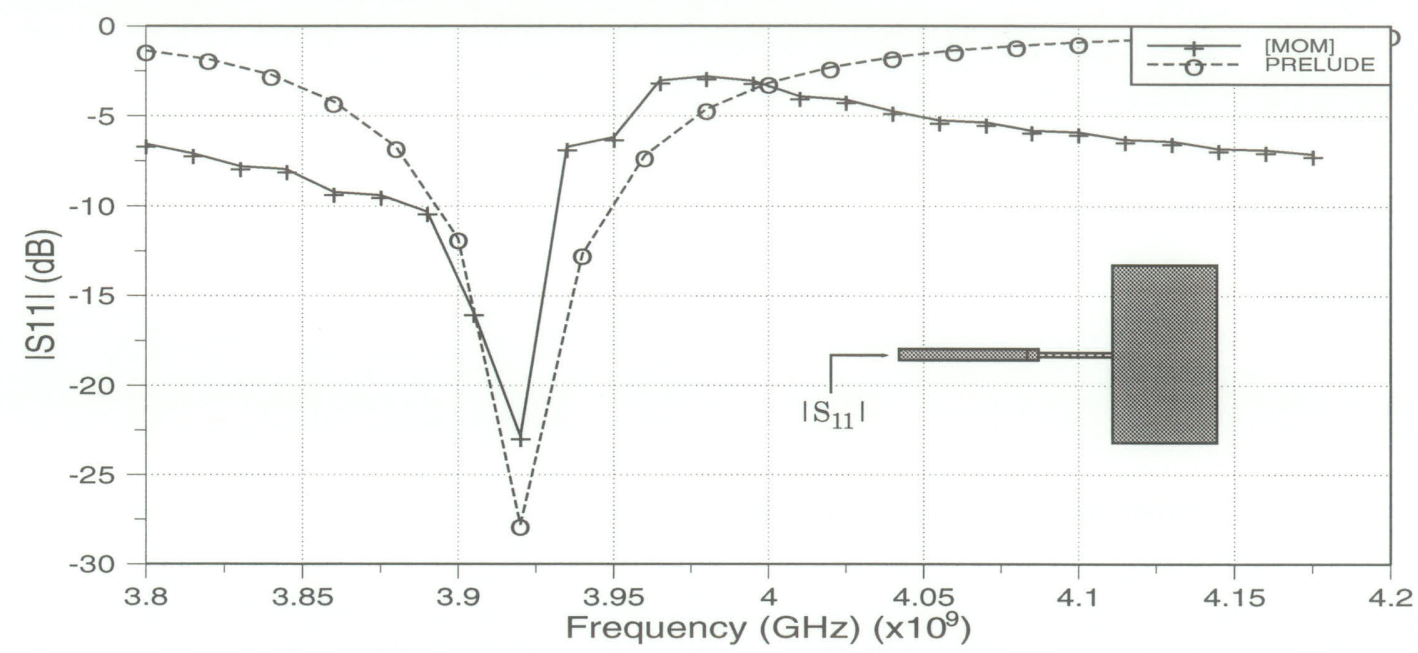

Figure 5.16 : Reflection coefficient of the matched antenna

From the graph we can see that the antenna is matched at the design frequency. The comparison between the two results show that on average there is a discrepancy of about $5 \mathrm{~dB}$ in the MPIE/MOM solution. Our calculation show that the antenna is matched quite well at the resonant frequency (less than -20dB) and it's a reasonable starting point for most antenna designs.

Also, the simulation run time for the MPIE/MOM solution is observed and provided in table 5.2 .

Table 5.2 : Run-time for MPIE/MOM solution (SPARC-5 Machine)

\begin{tabular}{|c|c|}
\hline Frequency points & $\begin{array}{c}\text { Total run time } \\
\text { (minutes) }\end{array}$ \\
\hline 7 & $7: 09$ \\
\hline 14 & $15: 03$ \\
\hline 21 & $23: 42$ \\
\hline
\end{tabular}

The above results are obtained for 57 current cells and the program is compiled under Unix environment in a Sparc 5 machine. No other processes were active during the running phase. Because of the unavailability of run time data for 
actual Sommerfeld Integral based MOM solution, comparison tests between the two methods could not be carried out. However, the data from the above table shows that our MPIE/MOM solution is reasonably efficient. The run-time efficiency also depends on the effectiveness of program coding as well as the computer capabilities. The program is written in FORTRAN and care is taken to make it as efficient as possible. However, this still does not guarantee the maximum efficiency that might be possible for the solution. Therefore, from our observations it is justifiable to mention that our MPIE/MOM solution is reasonably efficient.

\subsection{Circularly Polarized Microstrip Antenna.}

Circular polarization can be achieved in microstrip antennas by simply modifying the feed structure to produce two degenerate orthogonal modes. This will produce two orthogonally polarized waves and circular polarization is thus possible. In this section, a circularly polarized microstrip antenna is analyzed both theoretically and experimentally. The design is first verified numerically and a fabricated prototype is tested to verify the performance.

\subsubsection{Design, Simulation and Experimental Results .}

A wide variety of design methods are available to obtain circular polarization from patch antenna elements. In our case, we consider a simple design technique which involves the use of two separate and spatially orthogonal feeds excited with a

relative $\pm 90^{\circ}$ phase difference. A power splitter is used to supply power to the two 
feeds. The desired phase difference can be obtained by adding extra quarter wave line in one of the feed lines.

The antenna is designed to operate at $2.5 \mathrm{GHz}$. The dimensions of the patch is chosen such that it's less than a single wavelength (1.425 inches for our case) in both length and width. A fiberglass reinforced substrate is used as a ground plane, for which the dielectric constant is $\varepsilon_{r}=2.54$ and height is $1 / 8$ ". The substrate is considered to be very lossy and is not widely used for high performance microstrip antennas. However, for this case, it can be used to test the design parameters. Furthermore, fabrication is easier because of it's tough durability.

The basic configuration of this type of circularly polarized (CP) antenna includes a radiating element with two separate feeds originating from a single feed, as shown in Fig. 5.17 (i). A power divider is used to split power between the feeds.

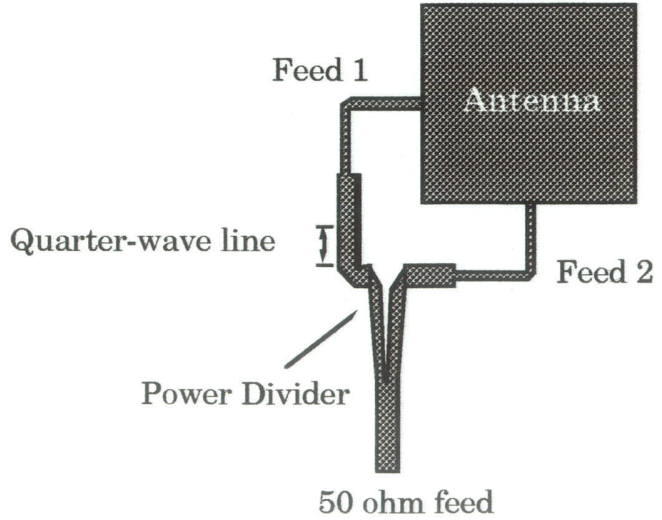

i. Design configuration

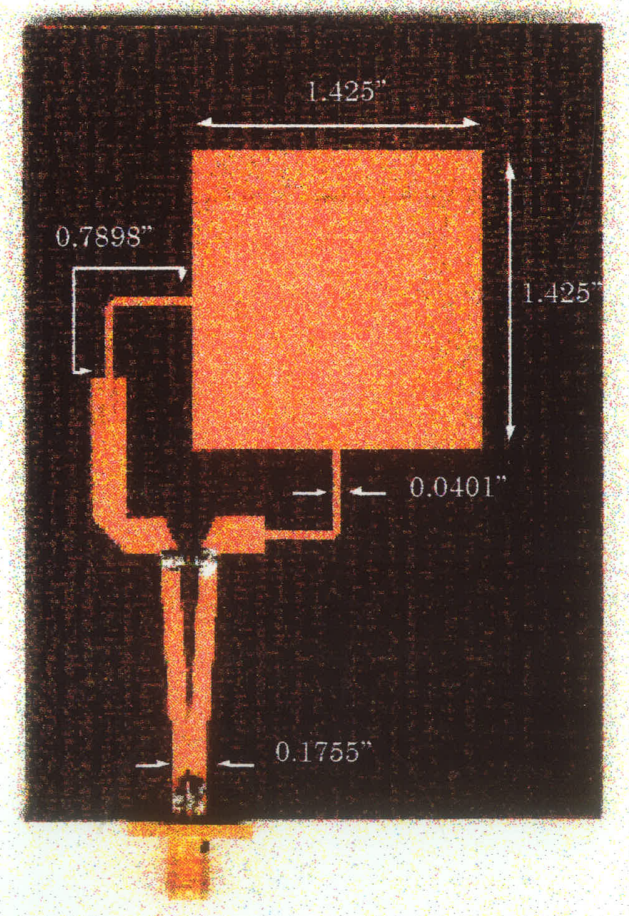

ii. Fabricated prototype

Figure 5.17 : Circularly polarized microstrip patch antenna 
In the design of the CP antenna, the first step is to calculate the input impedance of the radiating patch at the two feed points. Impedance values should be equal at these feed points, i.e. $\operatorname{Zin}_{11}=\operatorname{Zin}_{22}$. The input impedance results are given below in Fig. 5.18, for $2 G H z$ to $3 G H z$ frequency range.

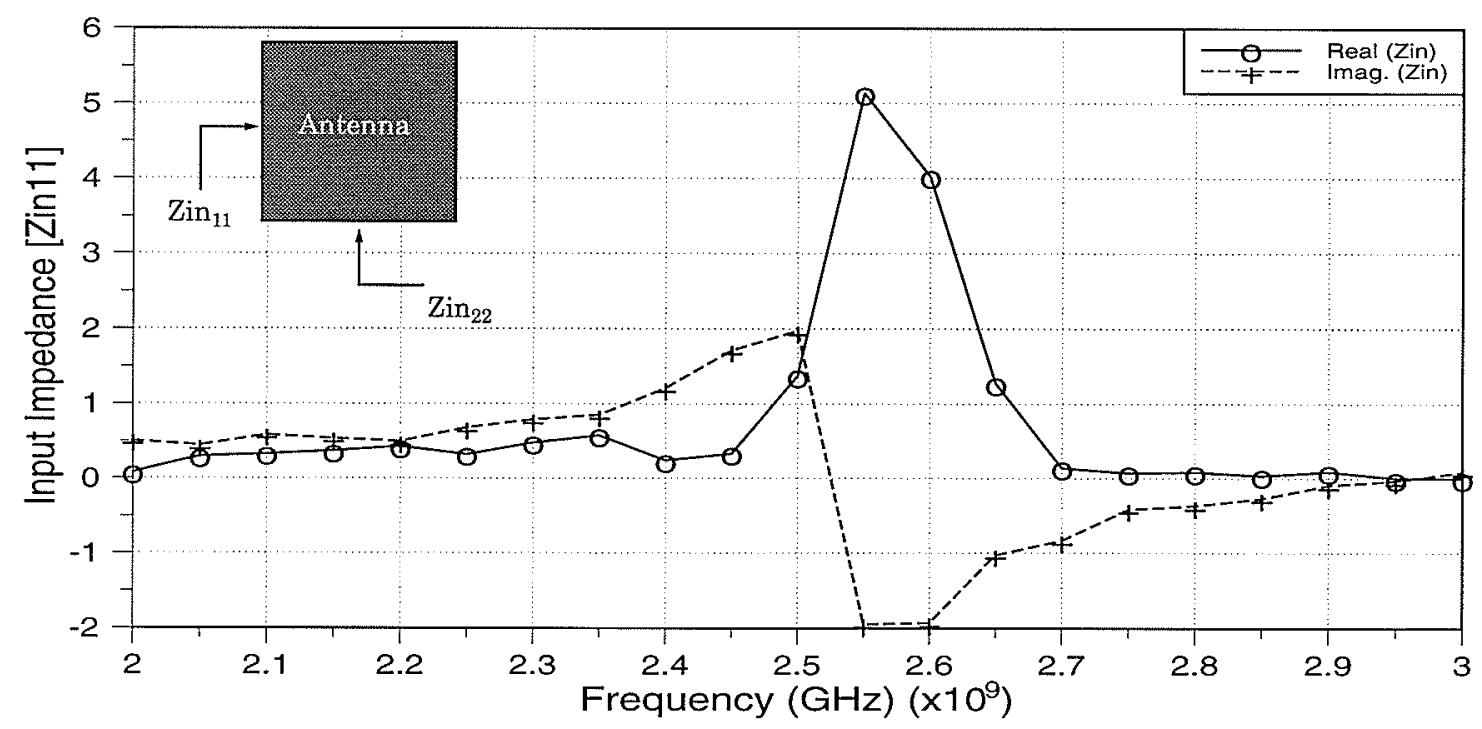

Figure $5.18:$ Normalized input impedance of the radiating element

Now a matching circuit is designed for the input impedance at resonant frequency. Since a $50 \Omega$ feed line is used, the antenna should be matched for this impedance. An extra quarter-wave line is added to one of the matching feed lines to achieve circular polarization. A simple power divider is used to split the power between the feeds [18]. The length of the main $50 \Omega$ feed line can be used as desired. The completed design is provided in Fig. 5.17(i). Irregular dimensions of the two feeds may lead to design discrepancies. This translates to a poor circular polarization from the antenna. Therefore, the two feedlines are carefully designed so as to obtain maximum performance.

The whole structure is simulated to extract the reflection coefficient. For a 2 
$\mathrm{GHz}$ to $3 \mathrm{GHz}$ frequency range, the forward reflection coefficient of the entire circuit is calculated and shown in Fig. 5.19. From the graph, we can see that the antenna is matched quite well near the design frequency. It seems that the resonant frequency has shifted a little bit, but it is still reasonably close to the design frequency.

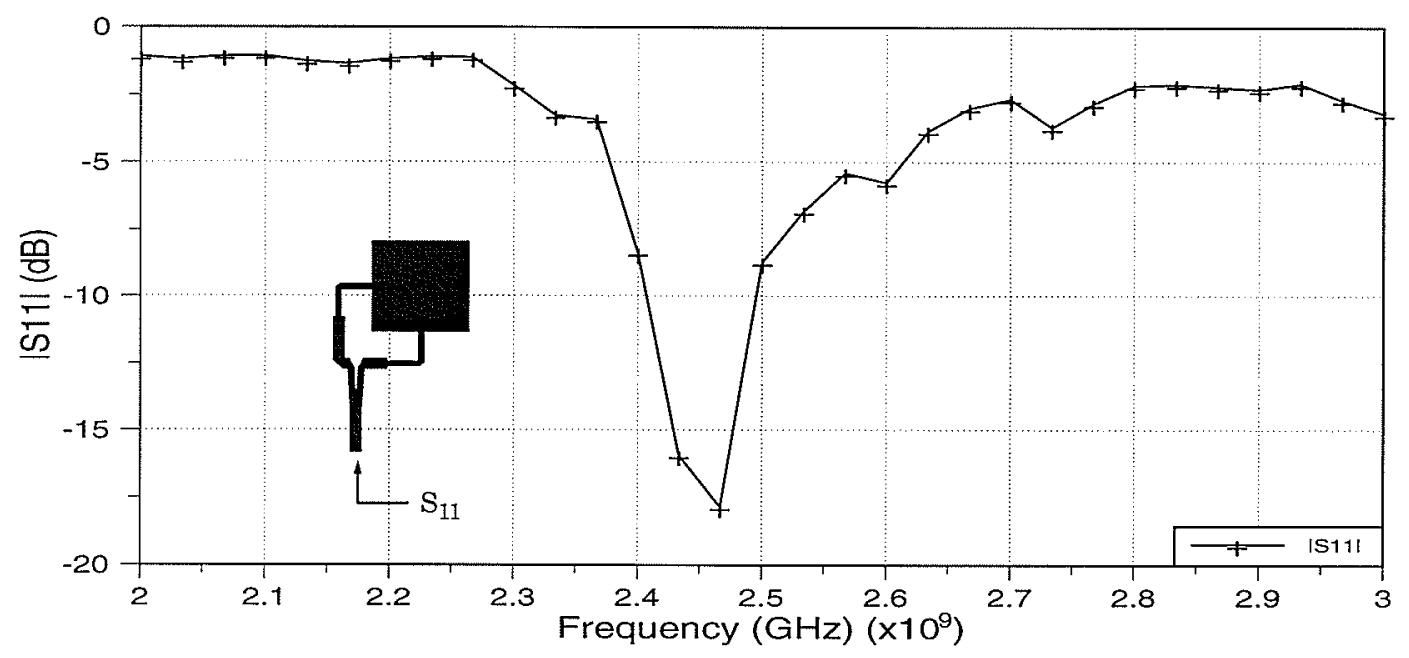

Figure 5.19 : Calculated reflection coefficient of the CP microstrip antenna

The antenna is fabricated using the milling machine provided by the Department of Electrical and Computer Engineering. The dimensions are first sketched in AutoCAD and then transferred to a software which controls the operation of the milling machine. The sketching of the whole antenna structure required extra caution mainly because the preciseness in the dimensions of the antenna as well as the feed structure is very important. This will determine the actual performance of the antenna. It took about 8 minutes to fabricate the whole antenna structure. The corresponding dimensions of the final prototype is very accurate with virtually no errors. A picture of the designed antenna is shown in Fig. 5.17(ii).

The reflection coefficient of the antenna is measured using a Network 
Analyzer. A $50 \Omega$ coaxial connector is connected to the main feed line and sweep for the entire frequency range ( $2 \mathrm{GHz}$ to $3 \mathrm{GHz}$ ) is shown in Fig. 5.20.
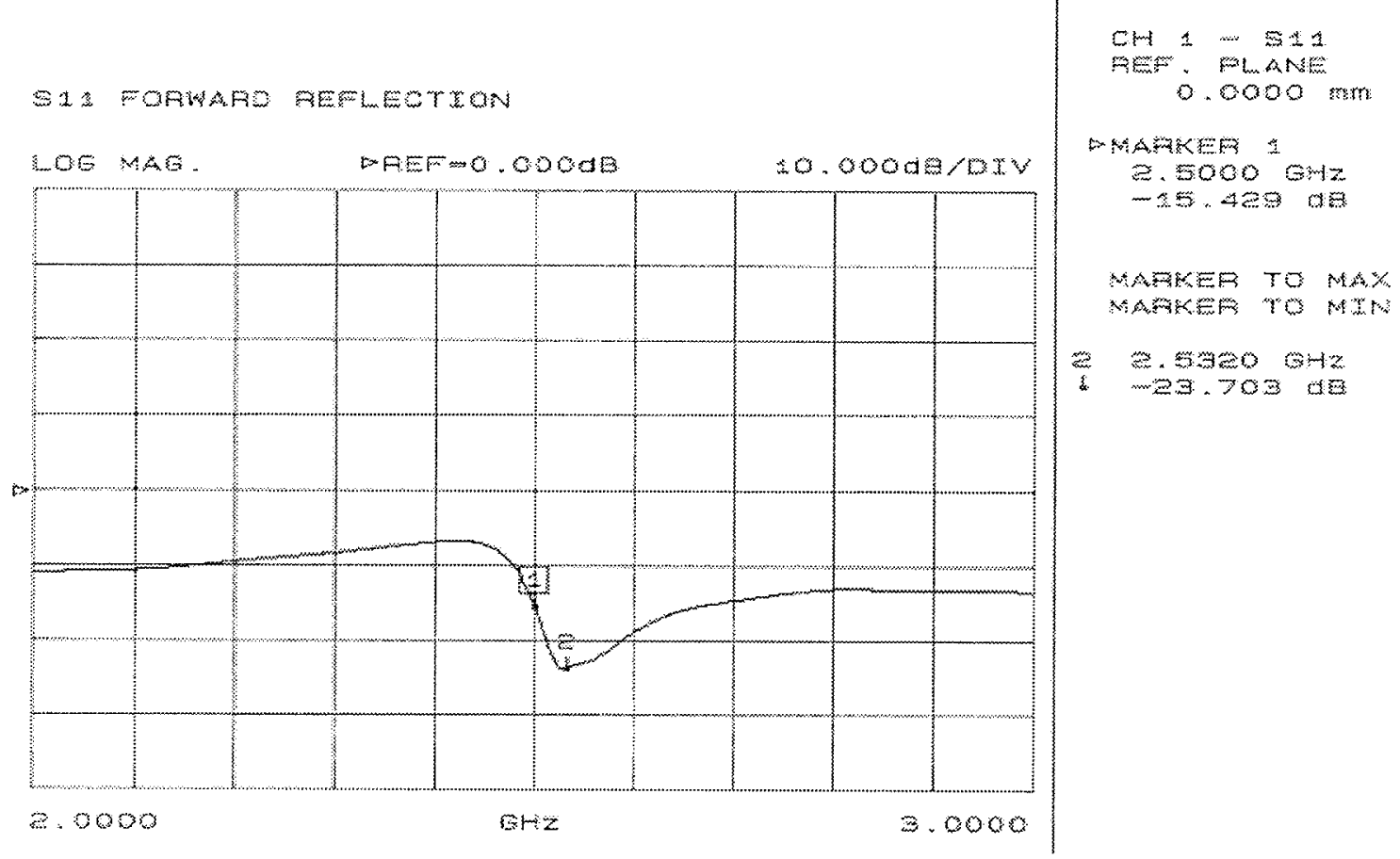

Figure 5.20 : Measured reflection coefficient of the CP microstrip antenna

We can see that the antenna is matched well around the design frequency. The resonant frequency is about $2.53 \mathrm{GHz}$ for the fabricated antenna. The antenna is now tested in the Anechoic chamber to measure far field radiation pattern. It's mounted as a receiver on a rotating shaft and a linearly polarized horn antenna is used as a transmitter. The transmitter is systematically rotated to obtain circular polarization. Due to the unavailability of a circularly polarized transmitter, the cross polar pattern could not be measured in the chamber.

The tests are carried out for the whole frequency range at certain intervals. We will look at two radiation pattern measurements, one at $2.5 \mathrm{GHz}$ and one at the resonant frequency, $2.54 \mathrm{GHz}$. These are shown in Fig. 5.21 and 5.22, respectively. 
THE UNIVERSTY OF MANTTOBA

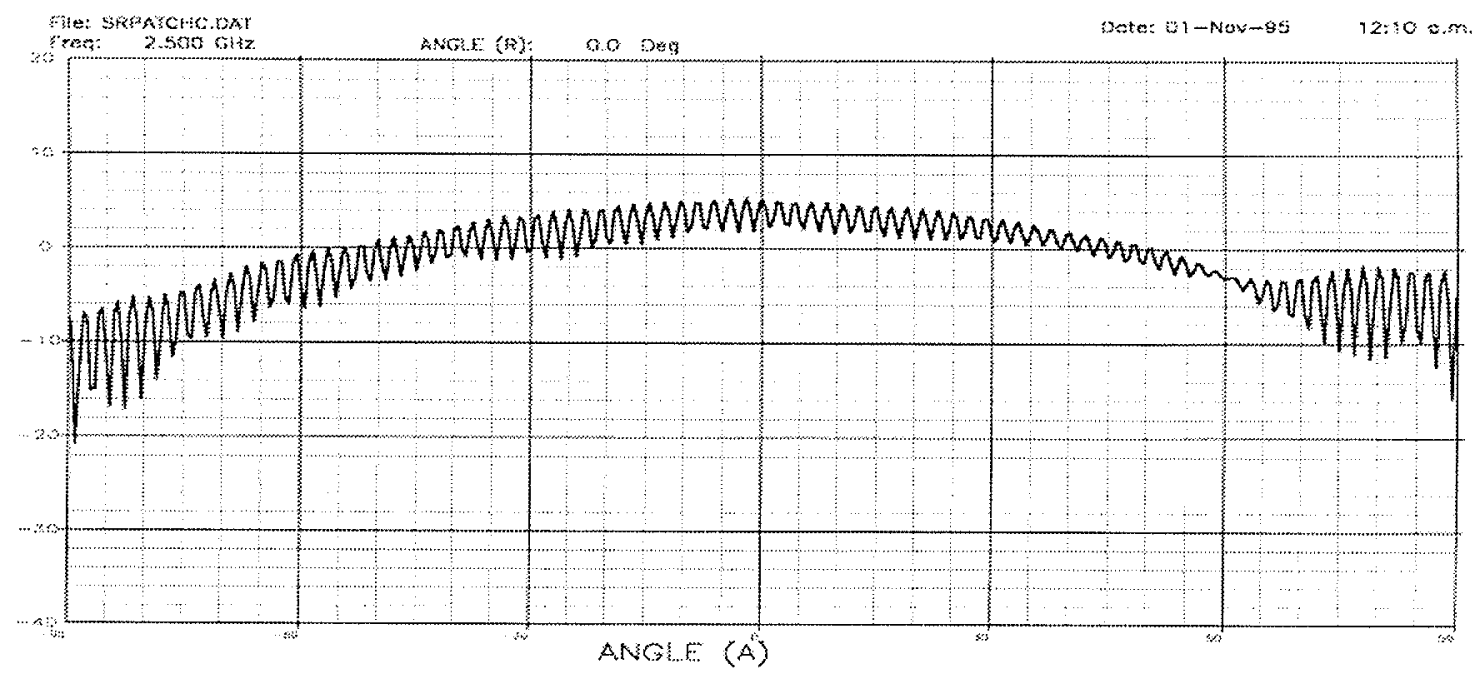

Figure 5.21 : Far-field pattern at $2.5 \mathrm{GHz}$

At $2.5 \mathrm{GHz}$, the measured gain is $6.77 \mathrm{~dB}$. Also, the measurement is not symmetric due to irregularity in the mounting platform and the transmitting tower.

THE UNIERSITY OF MANITOBA

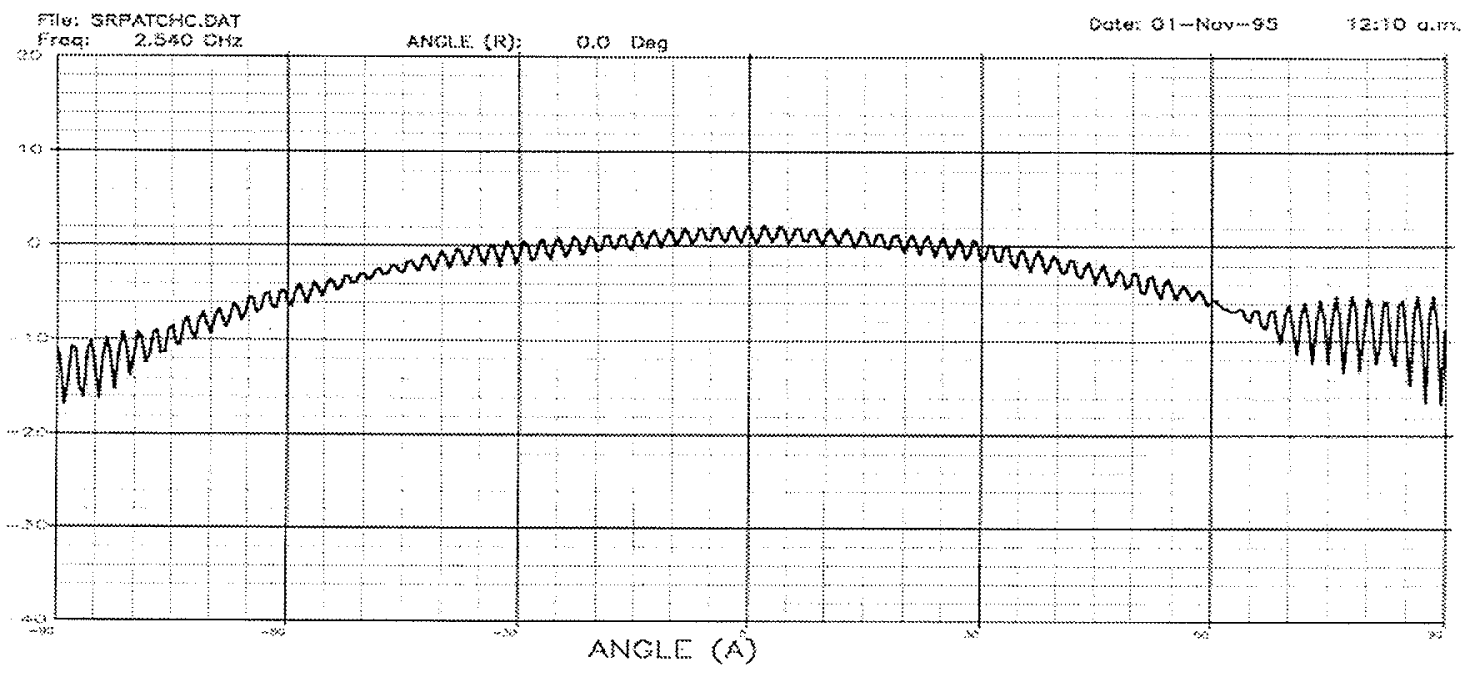

Figure 5.22 : Far-field pattern at $2.54 \mathrm{GHz}$

The final actual gain of the antenna at $2.54 \mathrm{GHz}$ is $4.27 \mathrm{~dB}$. Performance seems to be better at this frequency. As in the previous measurement, a slight 
irregularity is noticable in the pattern and it's due to irregularities in the mounting of the antenna.

In this chapter, we have proven that the approximate Green's function based MPIE/MOM solution is quite effective in predicting the performance of microstrip structures. We have compared our results with the published results and also with a full-wave analysis based software tool. A linearly polarized and a circularly polarized microstrip antenna are designed and analyzed. The experimental data for the circularly polarized antenna showed that the design characteristics are quite accurate. This further enforces the accuracy of the approximate Green's function based MPIE/MOM solution method. Errors visible in this method can be limited by avoiding the use of too many numerical approximations in the computation process. Introduction of approximations will definitely reduce run time but will result in an error dominated solution. Therefore, a compromise between efficiency and accuracy is the key to achieving a satisfactory and efficient design method. 


\section{Chapter VI}

\section{Conclusion}

An approximate Green's function technique in conjunction with the method of moments proves to be an efficient and powerful technique for the analysis of microstrip structures. Investigation into the accuracy and efficiency of this method have provided conclusive evidence that the procedure is quite reliable and compares well with established full-wave analysis techniques.

The thesis is divided into three main parts. First, a general derivation of approximate Green's function is presented as the starting point of the formulation. The Green's function is applied in the Mixed Potential Integral Equation (MPIE) to formulate the solution for microstrip structures. This required the use of boundary conditions and few theoretical assumptions. The Method of Moments technique is used to acquire solution from the MPIE. Extraction of surface currents from the MPIE/MOM solution provides the initial stage for the analysis. Further 
manipulation of this solution yields frequency dependent characteristics such as the S-parameters and far field radiation patterns.

Based on the theoretical analysis of the the Green's function method, a computer program has been developed to solve the MPIE/MOM solution. The results are compared with published numerical results as well as experimental results. In addition, the accuracy of the solution is weighed against the results obtained from a full-wave analysis based software tool and is found to be in good agreement. A half wavelength dipole is analyzed to test the convergence of the solution and to verify the solution in terms of input impedance calculations. The characteristic impedance and effective dielectric constant of a off-center fed microstrip transmission line are also calculated and compared with existing results. Design of a microstrip antenna and it's matching feed line is presented. Furthermore, a design of a circularly polarized microstrip antenna and the corresponding experimental results are provided.

There are few recommendations that might be useful for future research. Foremost is that a closed form solution to extract Green's function, instead of Sommerfeld integrals, might lead to an even more accurate and efficient method. Secondly, in the present simulation process, the circuit needs to be segmented into current cells first. If the structure to be analyzed is complicated, the segmentation process can be a very tedious and time consuming. Use of a tool to extract mesh coordinates will provide a less exhaustive simulation process and is recommended for future research.

The numerical method developed in this project is an efficient and reasonably accurate technique and it can be implemented to design microstrip circuits and antennas. Further research into this numerical approach may provide efficient design environment for high frequency circuit designers. 


\section{Bibliography}

[1] J. R. Mosig and F. E. Gardiol, "Analytical and numerical techniques in the Green's function treatment of microstrip antennas and scatterers", IEE Proc. Microwaves, Opt. and Antennas, Vol.130, Pt. H, pp. 175-182, March 1983.

[2] Edwin Kim-Leuk Yeung, Multilayer two port microstrip discontinuities, Master's thesis, Dept. of Electrical Engineering., Queen's University, Kingston, October 1992.

[3] J. R. Mosig and F. E. Gardiol, "General integral equation formulation for micrsotrip antennas and scatterers", IEE Proc. Microwaves, Opt. and Antennas, Vol.132, Pt. H, pp. 424-432, Dec. 1985.

[4] S. Couture, The method of moments applied to two layer microstrip structures, Master's thesis, Dept. of Electrical Engineering., Queen's University, Kingston, Aug. 1991.

[5] Y. L. Chow, J. J. Yang, D. G. Fang and G. E. Howard, "A closed form spatial Green's function for the thick microstrip substrate", IEEE Transactions on Microwave Theory and Techniques, Vol. 39, No. 3, pp. 588-592, March 1991.

[6] Juan R. Mosig and F. E. Gardiol, "A dynamical radiation model for microstrip structures", Advances in Electronics and Electron Physics, Vol. 59, London: Academic Press, 1982, pp. 139-239.

[7] C. A. Balanis, Advanced Engineering Electromagnetics, New York : John Wiley \& Sons, 1989. 
[8] J. R. James, P.S. Hall and C. Wood, Microstrip Antenna Theory and Design, London : Peter Peregrinus Ltd., 1981.

[9] R. F. Harrington, Time-Harmonic Electromagnetic Fields, New York : McGraw Hill, 1961.

[10] R. F. Harrington, Field Computation by Moment Methods, New York : McMillan, 1968.

[11] I. J. Bahl and P. Bhartia, Microstrip Antennas, Dedham : Artech House, 1980.

[12] K. R. Carver and J. W. Mink, "Microstrip antenna technology", IEEE Transactions on Antennas and Propagation, pp. 2-24, 1981.

[13] T. N. Judge, , Master's thesis, Department of Electrical and Computer Engineering, University of Manitoba,

[14] D. M. Pozar, "Input impedance and mutual coupling of rectangular microstrip antennas", IEEE Transactions on Antennas and Propagation, Vol. 30, pp. 1191-1196, 1982.

[15] R. K. Hoffman, Handbook of Microwave Integrated Circuits, Norwood : Artech House, 1987.

[16] K. C. Gupta, Microstrip Lines and Slotlines, Norwood : Artech House, 1979.

[17] J. R. Mosig, R. C. Hall and F.E. Gardiol, "Numerical analysis of microstrip patch antennas", Handbook of Microstrip Antennas, Vol. 1, London : Peter Peregrinus, 1990, pp. 393-454.

[18] Peter A. Rizzi, Microwave Engineering, Prentice Hall, 1988. 
[19] T. C. Edwards, Foundations for Microwave Circuit Design, John Wiley \& Sons, 1981.

[20] David K. Cheng, Field and Wave Electromagnetics, Addison-Wesley, 1989.

[21] E. K. Miller, L. M. Mitschang and E. H. Newman, Computational Electromagnetics, IEEE Press, 1991.

[22] T. Itoh and W. Menzel, "A full wave analysis method for open microstrip structures", IEEE Transactions on Antennas and Propagation, Vol. 29, pp. 63-68, Jan. 1981.

[23] S. Couture, J. C. Beal and Y. M. M. Antar, "The cubic spline interpolation of a standing-wave's envelope", IEEE AP-S International Symposium Conference Proceedings, July 1992.

[24] S. R. H. Hoole, Computer Aided-Analysis and Design of Electromagnetic Devices, New York : Elsevier Science, 1989.

[25] PRELUDE Design Version 1.0, Boulder Microwave Technologies Inc., 1993-1995. 


\section{Appendix A}

\section{Analytical Integration of Green's Function}

The dominant term in the vector and scalar potential Green's functions are

given by the corresponding static terms, $G_{\text {Astatic }}^{x x}$ and $G_{\text {Vstatic }}$

$$
\begin{gathered}
G_{\text {Astatic }}^{x x}=\frac{\mu_{o}}{4 \pi} \frac{1}{\left|r-r^{\prime}\right|} \\
G_{\text {Vstatic }}=\frac{1}{2 \pi \varepsilon_{o}\left(\varepsilon_{r}+1\right)} \frac{1}{\left|r-r^{\prime}\right|}
\end{gathered}
$$

For a current cell having the dimension $2 a \times b$, the final analytic integration expression for eqn. (3.1), without the constants, for a static term of the vector potential Green's function is given by [2]

$$
\begin{aligned}
& \frac{1}{a}\left[\left(a+x^{\prime}\right)^{2} C\left(\frac{2\left(a+x^{\prime}\right)}{b}\right)+\left(a-x^{\prime}\right)^{2} C\left(\frac{2\left(a-x^{\prime}\right)}{b}\right)-2\left(x^{\prime}\right)^{2} C\left(\frac{2 x^{\prime}}{b}\right)\right]+ \\
& \frac{1}{a}\left[\left(a+x^{\prime}\right) b S\left(\frac{2\left(a+x^{\prime}\right)}{b}\right)+\left(a-x^{\prime}\right) b S\left(\frac{2\left(a-x^{\prime}\right)}{b}\right)-2 b\left(x^{\prime}\right) S\left(\frac{2 x^{\prime}}{b}\right)\right]- \\
& \frac{b}{2 a}\left[\sqrt{\left(a+x^{\prime}\right)^{2}+\left(\frac{b}{2}\right)^{2}}+\sqrt{\left(a-x^{\prime}\right)^{2}+\left(\frac{b}{2}\right)^{2}}-2 \sqrt{\left(x^{\prime}\right)^{2}+\left(\frac{b}{2}\right)^{2}}\right] \\
& \text { where, } C(t)=\cosh ^{-1}(t) \text { and } S(t)=\sinh ^{-1}(t) \text {. Also, } x^{\prime} \text { is the location of }
\end{aligned}
$$
observation point. 
Similarly, from eqn. (3.2), the analytic expression for the vector potential static Green's function is given as [2]

$$
\begin{array}{r}
\left(\frac{a}{2}-x^{\prime}\right)\left[\ln \left(\sqrt{\left(\frac{a}{2}-x^{\prime}\right)^{2}+\left(\frac{b}{2}\right)^{2}}+\frac{b}{2}\right)-\ln \left(\sqrt{\left(\frac{a}{2}-x^{\prime}\right)^{2}+\left(\frac{b}{2}\right)^{2}}-\frac{b}{2}\right)\right]+ \\
\left(\frac{a}{2}+x^{\prime}\right)\left[\ln \left(\sqrt{\left(\frac{a}{2}+x^{\prime}\right)^{2}+\left(\frac{b}{2}\right)^{2}}+\frac{b}{2}\right)-\ln \left(\sqrt{\left(\frac{a}{2}+x^{\prime}\right)^{2}+\left(\frac{b}{2}\right)^{2}}-\frac{b}{2}\right)\right]+ \\
b\left[\ln \left(\sqrt{\left(\frac{a}{2}-x^{\prime}\right)^{2}+\left(\frac{b}{2}\right)^{2}}+\left(\frac{a}{2}-x^{\prime}\right)\right)-\ln \left(\sqrt{\left(\frac{a}{2}-x^{\prime}\right)^{2}+\left(\frac{b}{2}\right)^{2}}-\left(\frac{a}{2}-x^{\prime}\right)\right)\right]
\end{array}
$$

Where, as in the vector case, $x^{\prime}$ is the location of observation point. These solutions were rigorously studied by the author [ ] and was found to be accurate. 


\section{Appendix B}

\section{Derivations for $\mathrm{y}$ - directed Current}

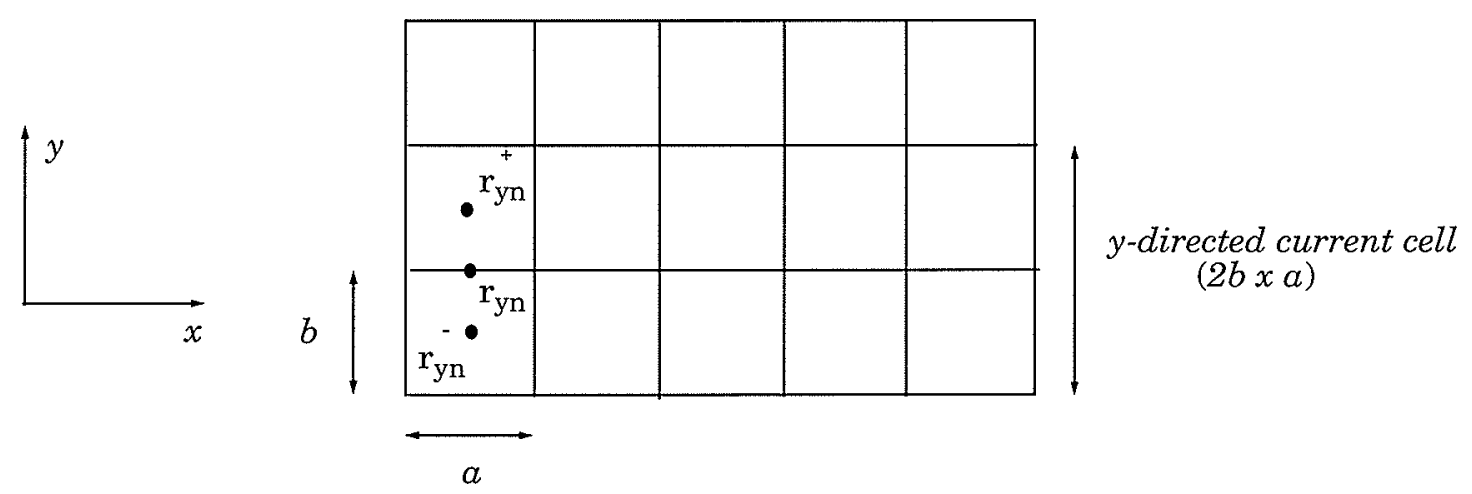

Figure B.1 : configuration for $y$-directed current cell

The y-directed surface current is expanded over a set of current basis function (2D triangular function) [3].

$$
J_{s y}=\frac{1}{a} \sum_{n=1}^{N-1} I_{y n} T_{y}\left(r-r_{y n}\right)
$$

where,

$$
T_{y}(r)= \begin{cases}l-\frac{|y|}{b} & |x|<\frac{b}{2}, \quad|y|<a \\ 0 & \text { elsewhere }\end{cases}
$$

Similarly, the surface charge density is given by, 


$$
\rho_{s}=\frac{1}{j \omega a b} \sum_{n=1}^{N-1} I_{y n}\left[R\left(r-r_{y n}^{+}\right)-R\left(r-r_{y n}^{-}\right)\right]
$$

where,

$$
R(r)= \begin{cases}1 & |x|<\frac{b}{2}, \quad|y|<\frac{a}{2} \\ 0 & \text { elsewhere }\end{cases}
$$

The vector potential $\Gamma_{A}$ and scalar potential $\Gamma_{V}$ are given by the following equations [3],

$$
\begin{aligned}
& \Gamma_{A}^{y y}\left(r \mid r_{n}^{\prime}\right)=\int_{s_{n}} \vec{G}_{A}^{y y}\left(r \mid r^{\prime}\right) T_{y}\left(r^{\prime}-r_{y n}\right) d r^{\prime} \\
& \Gamma_{V}\left(r \mid r_{o n}\right)=\int_{s_{y n}} G_{V}\left(r \mid r^{\prime}\right) R\left(r^{\prime}-r_{y n}\right) d r^{\prime}
\end{aligned}
$$

The tangential electric field is calculated from,

$$
E_{y}^{e}(r)=\frac{j w}{a} \sum_{n=1}^{N-1} I_{y n} \Gamma_{A}^{y y}\left(r \mid r_{n}{ }^{\prime}\right)+\frac{1}{j \omega a b} \sum_{n=1}^{N-1} I_{y n} \frac{\partial}{\partial y}\left[\Gamma_{V}\left(r \mid r_{n}{ }^{+}\right)-\Gamma_{V}\left(r \mid r_{n}{ }^{-}\right)\right]
$$

From the above expressions, y-directed current distribution can be calculated by the Method of Moments solution. 